\title{
Dynamic Strategic Information Transmission
}

\author{
Mikhail Golosov, Vasiliki Skreta, Aleh Tsyvinski, and Andrea Wilson*
}

September 16, 2011

\begin{abstract}
This paper studies strategic information transmission in a finite horizon environment where, each period, a privately informed expert sends a message and a decision maker takes an action. Our main contribution is to show that communication in this dynamic environment is drastically different from the canonical static environment. First, full revelation is possible. We provide a constructive method to build such an equilibrium. Second, we show that complicated communication, where far-away types pool, allows dynamic manipulation of beliefs and enables better information release in the future. If communication is restricted to be simple, such as monotonic partitional, full revelation is impossible. Thirdly, we show that conditioning future information release on past actions improves incentives and information release.
\end{abstract}

Keywords: asymmetric information; cheap talk; dynamic strategic communication; full information revelation.

${ }^{*}$ Golosov: Princeton and NES; Skreta: NYU, Stern School of Business; Tsyvinski: Yale and NES; Wilson: NYU. Golosov and Tsyvinski thank National Science Foundation and EIEF for hospitality. We thank Manuel Amador, Luca Andrelini, Sandeep Baliga, Abhijit Banerjee, Marco Battaglini, Andreas Blume, Adam Brandenburger, Amanda Friedenberg, Johannes Horner, Jerome Mathis, Ronny Razin, Larry Samuelson, and Andy Skrzypacz for inspiring discussions and comments. 


\section{Introduction}

The seminal paper on strategic information transmission by Crawford and Sobel (1982) is a model of choice for a number of applications, ranging from economics and political science, to philosophy and biology. ${ }^{1}$ In that paper, a biased and privately informed expert and a decision-maker interact only once. The conflict of interest results in coarse information revelation, and in some cases, in no information at all. We study strategic information transmission in a dynamic, finite horizon extension of the Crawford and Sobel setup. Each period the expert sends a message and a decision maker takes an action. Only the expert knows the state of the world which remains constant over time and the game. We maintain all other features of the Crawford and Sobel (1982) environment, in particular, that the interests of the expert and the decision maker differ.

There are many environments in which information transmission is dynamic. Many sequential decisions have to take place, and the decision maker seeks the expert's advice prior to each one of them. The earlier literature on dynamic communication has largely focused on the role of reputation, see, for example, Sobel (1985), Morris (2000), and Ottaviani and Sorensen (2006), (2006b). Our paper focuses on the dynamics of strategic communication.

Our most surprising, and difficult to establish finding, is to show that full information revelation is possible. We show this result in a challenging environment where the horizon is finite and both players are fully patient. Moving from one-shot to finite repetition often leaves the qualitative feature of equilibria unchanged: here we show an environment where finite repetition has a drastic impact. The construction of the fully revealing equilibrium relies on two key features. The first is the use of the separable groups: types who are far apart pool together initially, and then separate. As such, there is gradual revelation of information. The second feature is that the dynamic nature of the game enables the use of "trigger strategies," in which the expert promises better advice in the future if the decision maker chooses an action beneficial for the expert now. That is, trigger strategies are used to incentivize the decision maker, and to facilitate a gradual release of information that leads eventually to full revelation.

In a nutshell, in a multi-period interaction, communication can be facilitated by generating appropriate posteriors, and via the use of trigger strategies: the expert employs a signaling rule which makes future information revelation credible, but he only reveals the truth if his advice is followed early on. We now provide more details on the roles of separable groups and trigger strategies in our construction of a fully revealing equilibrium.

We first show that it is possible to divide all states into separable groups. A separable group is a set of types which are sufficiently far apart that each would rather reveal the truth, than mimic any other type in his group. Therefore, we create histories after which it is common knowledge that

\footnotetext{
${ }^{1}$ For a survey with applications across disciplines see Sobel (2008).
} 
the decision maker puts probability one on a particular separable group, at which point the types in this group separate. The division of all types into separable groups is quite delicate, because, given that there is a continuum of types, we need to form a continuum of such groups. The expert anticipates that if at some point he joins a separable group, then he will forgo his informational advantage. For the expert to join the separable group containing his true type, we have to make sure that he does not want to mimic a close-by type, who is supposed to join a different separable group. This is done via appropriate separation-inducing initial actions, which are not myopically optimal for the DM. The decision-maker is nevertheless willing to choose these actions, because the expert threatens to otherwise babble rather than revealing the truth.

We emphasize another necessary condition for full information revelation. The fully revealing equilibrium cannot be a monotonic partition equilibrium. In a monotonic partition equilibrium, the expert employs a uniform signaling rule where each type sends one message with probability one, and the set of types that choose the same message is connected. We prove that if attention is restricted to monotonic partition equilibria, learning stops. Moreover, we argue that non-monotonic equilibria can be strictly Pareto superior to all dynamic monotonic equilibria. Overall, we conclude that monotonic partition equilibria constitute a special class of equilibria in our dynamic setup, whereas this is a canonical class of equilibria in the static setup of Crawford and Sobel (1982). Non-monotonic (or non-convex) equilibria play a prominent role in Baliga and Sjőstrőm (2011), who highlight the role of pooling extremists. Such equilibria also appear in Baliga and Sjöstrőm (2008), who, building on Baliga and Sjőstrőm (2004), show how strategic ambiguity can reduce arm proliferation. Krishna and Morgan (2004) use non-monotonic equilibria in a static environment with mediated communication. In our dynamic environment the role of non-monotonic equilibria is to manipulate beliefs to affect incentives to release information over time.

Welfare properties of equilibria are another difference between the dynamic and the static strategic communication games. Crawford and Sobel (1982) show that, under some assumptions, both the expert and the decision maker ex-ante (before the state is realized) prefer equilibria with higher number of partitions. We provide an example that shows that it is not necessarily the case for dynamic equilibria. More partitions of the possible states of the world (i.e. larger number of equilibrium vectors of actions) do not guarantee higher expected payoff for the expert and the decision maker. The reason is as follows. In the dynamic setup the intetemporal nature of the indifference conditions that pin down the cutoffs that characterize partition equilibria allow for a much richer set of possibilities which overturn the static Pareto comparison results. A similar phenomenon occurs when the communication is noisy, as shown in an example of the working paper version of Blume, Board, and Kawamura (2007). In their example, a two-step partition Pareto dominates a three-step partition. We also present an example in which dynamic monotonic partition 
equilibria can be strictly Pareto superior to playing the most informative one-shot equilibrium in the first period, and babbling thereafter. Finally, we show that non-monotonic equilibria can be strictly Pareto superior to all dynamic monotonic equilibria.

Our work shows that the nature of dynamic strategic communication is quite distinct from its static counterpart. In the static case, because of the conflict of interest between the decisionmaker and the expert, nearby expert types have an incentive to pool together, precluding full information revelation. The single-crossing property also implies that in equilibrium, the action is a monotonic step function of the state. These two forces make complex signaling (even though possible) irrelevant, given that it is observationally equivalent to partitional signaling, and imply that only local incentive compatibility matters. In the dynamic setup the key difference is that today's communication sets up the stage for tomorrow's communication. Complex signaling helps in the dynamic setup, because it can lead to posteriors where possible states are sufficiently far apart that they have no incentive to mimic each other. Sustaining in equilibrium such posteriors requires complex signaling where one needs necessarily to worry about global incentive compatibility.

\section{Related Literature}

Crawford and Sobel (1982) is the seminal contribution on strategic information transmission. That paper has inspired an enormous amount of theoretical work and myriads of applications. Here we study a dynamic extension. Other papers that look at dynamic relations between one expert and one receiver differ from ours because, as already mentioned, their focus is on the sender's reputation. Other papers have a dynamic aspect because they allow for multi-round communication protocols, but with a single round of action(s). Aumann and Hart (2003) characterize geometrically the set of equilibrium payoffs when a long conversation is possible. In that paper, two players-one informed and one uninformed, play a finite simultaneous move game. The state of the word is finite, and players engage in direct (no mediator) communications (possibly infinitely long exchange of messages), before both choosing simultaneously costly actions. In contrast, in our model only the informed party sends cheap messages-the uniformed party chooses actions and the state is infinite. Krishna and Morgan (2004) add a long communication protocol to Crawford and Sobel (1982)'s game, whereas Goltsman Hőrner, Pavlov and Squintani (2009) characterize such optimal protocols. Forges and Koessler (2008a, 2008b) allow for a long protocol in a setup where messages can be certifiable. In all those papers once the communication phase is over, the decision maker chooses one action. In our paper, there are multiple rounds of communication and actions (each expert's message is followed by an action of the decision maker). A crucial difference from these earlier works is that in our environment the expert can condition his future advice on the decision-maker's past actions. 
In our setup, the dynamic nature of communication enables full information revelation. In contrast, full information revelation is not possible in the dynamic setup of Andrelini, Gerardi, and Lagunoff (2008) who consider dynamic strategic communication is a dynastic game and show that if preferences are not fully aligned "full learning" equilibria do not exist. Renault, Solan, and Vieille (2011) examine dynamic sender-receiver games, in a setup where the information is not fully persistent and the state is finite. They restrict attention to Markov equilibria and characterize the set of equilibrium payoffs. In contrast, we assume fully persistent information, finite horizon, and a state drawn from a continuum, and focus mainly on non-markovian equilibria. ${ }^{2}$

Our model bears some similarities to the static strategic communication with multiple receivers. In those models the expert cares about a sequence of actions, but in contrast to our model, those actions are chosen by different individuals. An important difference is that in our model the receiver cares about the entire vector of actions chosen, compared to those models where each receiver cares only about his own action. Still, some of the properties of the equilibria that we obtain also appear in the models with multiple receivers. For example, our non-monotonic example presented in Section 4 resembles Example 2 of Goltzman and Pavlov (2008). It is also similar to Example 2 in Krishna and Morgan (2004). ${ }^{3},{ }^{4}$

Full information revelation is possible in other variations of the Crawford and Sobel (1982) setup: When the decision maker consults two experts as in Battaglini (2002), Eso and Fong (2008) and Ambrus and $\mathrm{Lu}(2010) ;^{5}$ when information is completely or partially certifiable, as in Mathis (2008); and when there are lying costs and the state is unbounded as in Kartik, Ottaviani and Squintani (2007). In the case of multiple experts, playing one against the other is the main force that supports truthful revelation. In the case of an unbounded state, lying costs become large and support the truth. In the case of certifiable information, one can exploit the fact that messages are state-contingent to induce truth telling. All these forces are very different from the forces behind our fully revealing construction.

\footnotetext{
${ }^{2}$ Ivanov (2011) allows for a dynamic communication protocol in a setup where the expert is also initially uninformed and the decision maker controls the quality of information available to the expert. He builds on our construction and employs separable groups, but in a much different informational setting. (The DM gives the uninformed expert a device that initially reveals (to the expert only) the separable group containing the truth, and contains a built-in threat to only reveal the exact state if the expert reports this information truthfully).

${ }^{3}$ Equilibria can be non-monotonic also in environments where the decision maker consults two experts as in Krishna and Morgan (2001).

${ }^{4}$ For the expert, inducing a sequence of actions is payoff-equivalent to inducing a probability distribution over actions, so our analysis for the expert would go through also in such settings. Our set-up is however more flexible on the DM's side, since conditioning advice on past actions (trigger strategies), is impossible in mediated static set-ups.

${ }^{5}$ But full information is not possible in the setup of Levy and Razin (2007).
} 


\section{The Environment}

We extend the classical model of Crawford and Sobel (1982) to a dynamic setting. There are two players: an Expert - E (sender) and a Decision Maker - DM (receiver) who interact for finitely many periods $t=1, \ldots, T$. The expert knows the state of the world $\theta \in[0,1]$ which is constant over time and is distributed according to the c.d.f. $F$. At the beginning of each period $t$, the expert sends a (possibly random) message $m_{t}$ which depends on the realization of the state $\theta$ to the decision maker. The decision maker then updates his beliefs about the state and chooses an action $y_{t} \in \mathbb{R}$ that affects both players' payoffs. There is a conflict of interest because, for all states $\theta$, the expert's ideal choice of action differs from the decision maker's. This is captured by a scalar $b$, the bias parameter of the expert. The discount factor of the expert and the decision maker is $\delta_{i} \in[0,1]$ for $i \in\{E, D M\}$. When the state is $\theta$ and the decision maker chooses actions $y^{T}=\left(y_{1}, . ., y_{T}\right)$, the expert's payoff is given by

$$
U^{E}\left(y^{T}, \theta, b\right)=\Sigma_{t=1}^{T} \delta_{E}^{t-1} u^{E}\left(y_{t}, \theta, b\right)
$$

and the decision maker's payoff is given by

$$
U^{D M}\left(y^{T}, \theta\right)=\Sigma_{t=1}^{T} \delta_{D M}^{t-1} u^{D M}\left(y_{t}, \theta\right)
$$

We assume that period payoffs $u^{E}\left(y_{t}, \theta\right)$ and $u^{D M}\left(y_{t}, \theta, b\right)$ satisfy the conditions imposed by Crawford and Sobel (1982), namely that (i) $u^{i}$ is twice differentiable, for all $\theta$; (ii) $\frac{\partial u^{i}(y, \theta)}{\partial y}=0$ for some $y$ for $i \in\{E, D M\}$; (iii) that $\frac{\partial^{2} u^{i}(., .)}{\partial y^{2}}<0$; and (iv) a single crossing condition, $\frac{\partial^{2} u^{i}(., .)}{\partial \theta \partial y}>0$. Conditions (ii) and (iii) guarantee a unique maximum in $y$.

Let $y^{D M}(\theta)$ and $y^{E}(\theta)$ denote, respectively, the decision maker's and the expert's most preferred action when the true state $\theta$ is known by both players . The single crossing condition ensures that $y^{D M}(\theta)$ and $y^{E}(\theta)$ are increasing in $\theta$. We assume that, regardless of the state $\theta$, a conflict of interest exists: $y^{D M}(\theta) \neq y^{E}(\theta)$, for all $\theta$.

The decision maker observes his payoffs only at the end of the game. He does not observe his payoff at each period $t$. Otherwise, the problem would be trivial because the decision maker can learn the expert's information by simply inverting his payoff.

A strategy profile $\sigma=\left(\sigma_{i}\right)_{i=E, D M}$, specifies a strategy for each player. Let $h_{t}$ denote a history that contains all the reports submitted by the expert $m^{t-1}=\left(m_{1}, \ldots, m_{t-1}\right)$, and all actions chosen by the decision maker $y^{t-1}=\left(y_{1}, \ldots, y_{t-1}\right)$ up to, but not to including stage $t$. The set of all feasible histories at $t$ is denoted by $H_{t}$. A behavioral strategy of the expert, $\sigma_{E}$, consists of a sequence of signaling rules that map $[0,1] \times H_{t}$ to a probability distribution over reports $\mathcal{M}$. Let $q\left(m \mid \theta, h_{t}\right)$ denote the probability that the expert reports message $m$ at history $h_{t}$ when his type is $\theta$. A strategy for the decision maker, $\sigma_{D M}$, is a sequence of maps from $H_{t}$ to actions. We use 
$y_{t}\left(m \mid h_{t}\right) \in \mathbb{R}$ to denote the action that the decision maker chooses at $h_{t}$ given a report $m$. A belief system, $\mu$, maps $H_{t}$ to the set of probability distributions over $[0,1]$. Let $\mu\left(\theta \mid h_{t}\right)$ denote the decision maker's beliefs about the experts's type after a history $h_{t}, t=1, \ldots, T$. A strategy profile $\sigma$ and a belief system $\mu$ is an assessment. We seek strategy profiles and belief systems that form Perfect Bayesian Equilibria, (PBE).

In the paper we use the terminology as follows.

Definition 1 An equilibrium is called babbling if for all $m$ with $q\left(m \mid \theta, h_{t}\right)>0$, all $\theta \in[0,1]$, all $h_{t}$ and $t$, we have that $y_{t}\left(m \mid h_{t}\right)=\hat{y}$.

In other words, we call an equilibrium babbling, if the same action is induced with probability one for all states $\theta \in[0,1]$ and all $t \in T$.

Definition 2 We call a signaling rule $q$ uniform, if $q\left(m \mid \theta, h_{t}\right)$ is uniform, with support on $\left[\theta_{i}, \theta_{i+1}\right]$ if $\theta \in\left[\theta_{i}, \theta_{i+1}\right]$.

Definition 3 A partition equilibrium is one in which, at each period $t$ and history $h_{t}$, the expert employs only uniform signaling rules.

In other words, at a partition equilibrium, the expert follows a pure strategy in which, for any message $m$, the set of types sending message $m$ is connected.

Definition 4 An equilibrium is fully revealing if there exists a $\hat{T} \leq T$ such that after all histories along the equilibrium path leading to $\hat{T}$, the expert sends for all $\theta \in[0,1]$ a different message with probability one, inducing $y_{t}(\theta)=y^{D M}(\theta)$ from $t=\hat{T}$ on.

\section{$3 \quad$ Uniform Signaling}

We first briefly summarize the static cheap talk game of Crawford and Sobel (1982) in which uniform signaling rules are the canonical form of communication in static games. We then study properties of uniform signaling in our dynamic setup.

\subsection{The Canonical Static Communication}

We start by reviewing the Crawford and Sobel (1982) findings. For the static communication and decision game they show that all equilibria are equivalent to partition equilibria. The expert employs simple uniform signaling rules. He follows a pure strategy in which, for any message $m$, the set of types sending message $m$ is connected. This type of communication induces actions which are increasing step functions of the state. Hence, intervals of types pool together by sending 
the same message, ultimately, inducing the same action by the decision maker. This implies that communication is coarse. Even though the state $\theta$ takes a continuum of values, only finitely many different actions are induced.

The intuition behind this result can be summarized as follows. Fix an equilibrium of the oneshot game and let $y(\theta)$ denote an action induced when the state is $\theta$. The conflict of interest between the expert and the decision maker $\left(y^{D M}(\theta) \neq y^{E}(\theta)\right.$, for all $\left.\theta\right)$ implies that there are at most finitely many actions induced. The fact that $u^{E}$ is strictly concave in $y$, together with single-crossing and the finiteness of actions, implies that there are at most finitely many states $\theta$ where the expert is indifferent between two induced actions. Then, $y(\theta)$ can be, without loss of generality, taken to be single-valued. Because $y$ takes at most finitely many values, it is an increasing step function. The state space is divided into a finite number of subintervals, and $y$ takes a different value in each subinterval. Importantly, Crawford and Sobel (1982) show that, without loss of generality, equilibrium induced actions, $y$ can be taken to arise from uniform signaling rules. This result follows from the observation that all messages inducing the same action $y$ can be replaced by a single message. Therefore, more complex signaling rules play no role in the static setup.

\subsection{Uniform Signaling: A Special Kind of Dynamic Communication}

We now focus on simple partitional communication protocols (uniform signalling) and study their properties in our dynamic setup. We show two results. The first result is that with monotonic partition equilibria the decision maker never learns the truth. The second result establishes that when the bias is so large that only one action is induced at an equilibrium of the static game, the same is true also in dynamic monotonic partition equilibria. Finally, an example shows that dynamic partitions can differ and can be more informative compared to static ones.

In constructing partition equilibria, the main difference from the static case is that the expert's indifference conditions that characterize the cutoffs of the partitions are intertemporal. This makes the explicit solution of the resulting difference equation difficult even in simple environments, such as in the uniform quadratic loss case. To overcome this difficulty, we notice that at some point in the game the expert's indifference conditions reduce to the static ones and then apply the results of Crawford and Sobel (1982).

Using this approach, we first show that among the class of monotonic partition equilibria, there exists no equilibrium in which the decision maker eventually learns the state of the world for all $\theta \in[0,1]$. That is, there exist no fully revealing equilibria.

Proposition 1 For all $T$, there exist no fully revealing monotonic partition equilibria.

This result follows almost immediately from Crawford and Sobel (1982). A short sketch of the argument is as follows. Suppose, by contradiction, that there exists a fully revealing monotonic 
partition equilibrium. Then, there exists a period $\hat{T} \leq T$ in which the last subdivision occurs, with $y_{t}(\theta)=\theta$, for all $t \geq \hat{T}$. Since $y_{t}(\theta)=\theta$ (respectively $y_{t}(\theta+\varepsilon)=\theta+\varepsilon$ ) from $\hat{T}$ onwards, the incentive constraint at time $\widehat{T}$ for type $\theta$ to not mimic type $\theta+\varepsilon$ is

$$
\left(1+\delta+\delta^{2}+\ldots+\delta^{T-\hat{T}-1}\right) u^{E}\left(y_{t}(\theta), \theta\right) \geq\left(1+\delta+\delta^{2}+\ldots+\delta^{T-\hat{T}-1}\right) u^{E}\left(y_{t}(\theta+\varepsilon), \theta\right),
$$

and similarly for type $\theta+\varepsilon$. These conditions are equivalent to the static equilibrium conditions in Crawford and Sobel (1982), who proved that they imply that at most finitely many actions can be induced at an equilibrium of a static game, a contradiction to full revelation.

We now proceed to show that if all static equilibria are equivalent to babbling then all dynamic monotonic partition equilibria are equivalent to babbling.

Proposition 2 If all static equilibria are equivalent to the babbling equilibrium, then all dynamic monotonic partition equilibria are equivalent to babbling.

Proof. See Appendix A.

Note that the logic of the arguments used to establish Propositions 1 and 2 applies also to an infinite horizon environment.

It is also easy to see that the payoffs of both the expert and the decision maker of any static equilibrium can be achieved as a per period payoffs in the dynamic communication game. We can construct an "equivalent" (in terms of payoffs) dynamic equilibrium as follows: In the first period, all types follow the same strategies as in the static equilibrium. For all $t>1$ experts of all types "babble", and the decision maker repeats the same action she took in period 1 . It is trivial to verify that these strategies constitute an equilibrium.

In Appendix B, we show an example when in our dynamic setup $(T=2)$ both players can be strictly better-off compared to the best static equilibrium. In that example, $\delta_{E}=\delta_{D M}=1$, the state is uniformly distributed on $[0,1]$ and preferences satisfy

$$
u^{E}(y, \theta)=-(y-\theta-b)^{2} \text { and } u^{D M}(y, \theta, b)=-(y-\theta)^{2}
$$

with $b=\frac{1}{12}$. At the most informative static equilibrium the state space is divided in two subintervals: $\left[0, \frac{1}{3}\right]$ and $\left[\frac{1}{3}, 1\right]$ inducing two actions $\frac{1}{6}$ and $\frac{4}{6}$. We show that when $T=2$, there exists a partition equilibrium where the state space is divided ultimately in three sub-intervals: [0, 0.25], $[0.25,0.45833]$ and $[0.45833,1]$.

However, in dynamic settings it is also possible to have equilibria with more partitions that are inferior to ones with less (we present an example of such an equilibrium in Appendix C). This happens because a larger ultimate number of partitions may require extensive pooling earlier on, inducing overall lower welfare. This finding is in contrast to Crawford and Sobel (1982), who show 
that under their Assumption M equilibria can be easily Pareto ranked: both the expert and the decision maker ex-ante (before the state is realized) prefer the equilibrium with the highest number of partitions and actions induced. ${ }^{6}$ Our findings suggest that Pareto comparisons in dynamic cases are less straightforward, even if we restrict attention to monotonic partitional equilibria.

We proceed to study the role of complex signalling in our dynamic game.

\section{An Example with Complex Signaling and Dynamic Information Revelation}

In this section we present an example where the expert employs a complex signalling rule that induces an action of the decision maker that is not monotonic in the state. We show that, for a range of biases, this equilibrium is superior to all monotonic ones.

In the example below, the bias is so severe that if communication and the decision were static (one round), as in Crawford and Sobel (1982), all equilibria would be babbling. We show, that even in these extreme bias situations, some information can be revealed with just two rounds. This equilibrium has the feature that the decision maker learns the state quite precisely when the news is either horrific or terrific, but remains agnostic for intermediate levels.

Example 1 Dynamic equilibria can be non-monotonic

Consider a two period game where $\delta_{E}=\delta_{D M}=1$, types are uniformly distributed on $[0,1]$ and preferences are given by (1). We will construct an equilibrium with the following "piano teacher" interpretation: a child's parent (the DM) wants the amount of money he spends on lessons to correspond to the child's true talent $\theta$, whereas the piano teacher (expert) wants to inflate this number. In our equilibrium, parents of children who are at either the bottom or top extreme of the talent scale get the same initial message, "you have an interesting child" ( $m_{1(1)}$ below), and then find out in the second period whether "interesting" means great $\left(m_{2(3)}\right)$ or awful $\left(m_{2(1)}\right)$; parents of average children are told just that in both periods. More precisely, let the expert use the following signaling rule:

In period 1 , the expert for states in $[0, \underline{\theta}) \cup(\bar{\theta}, 1]$ sends a message $m_{1(1)}$ with probability 1 . For states in $[\underline{\theta}, \bar{\theta}]$, he sends a message $m_{1(2)}$ with probability 1 . In period 2 , the expert adopts the following signaling rule. For states in $[0, \underline{\theta})$ the expert sends a message $m_{2(1)}$ with probability 1 , for states in $[\underline{\theta}, \bar{\theta}]$ he sends a message $m_{2(2)}$ with probability 1 , and for states in $(\bar{\theta}, 1]$ he sends a message $m_{2(3)}$ with probability 1 . To sum up, we look for an equilibrium with the following

\footnotetext{
${ }^{6}$ The equilibrium with the largest number of partitions is the only equilibrium that satisfies the "no incentive to separate" (NITS) condition (Chen, Kartik and Sobel (2008)).
} 
signaling rule:

types in $[0, \underline{\theta})$ send message sequence $A=\left(m_{1(1)}, m_{2(1)}\right)$,

types in $[\underline{\theta}, \bar{\theta}]$ send message sequence $B=\left(m_{1(2)}, m_{2(2)}\right)$,

types in $(\bar{\theta}, 1]$ send message sequence $C=\left(m_{1(1)}, m_{2(3)}\right)$.

With this signaling rule, the optimal actions of the decision maker for periods 1 and 2 are, respectively, given by:

$$
\begin{aligned}
& t=1: y_{1(1)}=\frac{\underline{\theta}^{2}-\bar{\theta}^{2}+1}{2(\underline{\theta}-\bar{\theta}+1)} ; y_{1(2)}=\frac{\underline{\theta}+\bar{\theta}}{2} . \\
& t=2: y_{2(1)}=\frac{\theta}{2} ; y_{2(2)}=\frac{\underline{\theta}+\bar{\theta}}{2} ; y_{2(3)}=\frac{1+\bar{\theta}}{2} .
\end{aligned}
$$

After any out-of-equilibrium message the decision maker assigns probability 1 to the state belonging to $[\underline{\theta}, \bar{\theta}]$, and so will choose action $y^{\text {out }}=\frac{\theta+\bar{\theta}}{2}$. With these out-of equilibrium beliefs, no type of the expert has an incentive to send an out-of-equilibrium message.

In order for this to be an equilibrium, type $\underline{\theta}$ must be indifferent between message sequence $A$ and $B$ :

$$
-\left(\frac{\underline{\theta}^{2}-\bar{\theta}^{2}+1}{2(\underline{\theta}-\bar{\theta}+1)}-\underline{\theta}-b\right)^{2}-\left(\frac{\underline{\theta}}{2}-\underline{\theta}-b\right)^{2}=-2\left(\frac{\underline{\theta}+\bar{\theta}}{2}-\underline{\theta}-b\right)^{2}
$$

and type $\bar{\theta}$ must be indifferent between message sequence $B$ and $C$ :

$$
-\left(\frac{\underline{\theta}^{2}-\bar{\theta}^{2}+1}{2(\underline{\theta}-\bar{\theta}+1)}-\bar{\theta}-b\right)^{2}-\left(\frac{1+\bar{\theta}}{2}-\bar{\theta}-b\right)^{2}=-2\left(\frac{\underline{\theta}+\bar{\theta}}{2}-\bar{\theta}-b\right)^{2}
$$

At $t=2$ it must also be the case that type $\underline{\theta}$ prefers $m_{2(1)}$ to $m_{2(3)}$, and the reverse for type $\bar{\theta}$ : that is $-\left(\frac{\theta}{2}-\underline{\theta}-b\right)^{2} \geq-\left(\frac{1+\bar{\theta}}{2}-\underline{\theta}-b\right)^{2}$ and $-\left(\frac{1+\bar{\theta}}{2}-\bar{\theta}-b\right)^{2} \geq-\left(\frac{\theta}{\overline{2}}-\bar{\theta}-b\right)^{2}$. The global incentive compatibility constraints, requiring that all types $\theta<\underline{\theta}$ prefer sequence $A$ to $B$ and that all types $\theta>\bar{\theta}$ prefer $C$ to $B$, reduce to a requirement that the average induced action be monotonic, which is implied by indifference constraints $(2),(3) .^{7}$

A solution of the system of equations (2) and (3) gives an equilibrium if $0 \leq \underline{\theta}<\bar{\theta} \leq 1$. We solved this system numerically and found that the highest bias that makes this non-monotonic equilibrium

\footnotetext{
${ }^{7}$ Rearranging (3), the LHS is greater than the RHS for type $\theta$ (so he prefers $C$ to $B$ ) iff $(\theta-\bar{\theta})\left(\frac{y_{1(1)}+y_{2(3)}}{2}-y_{1(2)}\right)>0$, so we need $\frac{y_{1(1)}+y_{2(3)}}{2}>y_{1(2)}$ for this to hold $\forall \theta>\bar{\theta}$. This is implied by (3): adding $2\left(\frac{y_{1(1)}+y_{2(3)}}{2}-\bar{\theta}-b\right)^{2}$ to both sides and factoring yields $\left(y_{1(2)}-\bar{\theta}-b\right)^{2}-\left(\frac{y_{1(1)}+y_{2(3)}}{2}-\bar{\theta}-b\right)^{2}=\left(\frac{y_{1(1)}-y_{2(3)}}{2}\right)^{2} \geq$ 0 , so we need $\left|y_{1(2)}-\bar{\theta}-b\right| \geq\left|\frac{y_{1(1)}+y_{2(3)}}{2}-\bar{\theta}-b\right|$; since $y_{1(2)}<\bar{\theta}+b$, this implies $y_{1(2)} \leq \frac{y_{1(1)}+y_{2(3)}}{2}$, as desired. And similarly at $\underline{\theta}$.
} 
survive is $b=0.256$. For $b=0.256$, we obtain $\underline{\theta}=0.0581, \bar{\theta}=0.9823$. The corresponding optimal actions for period 1 are: $y_{1(1)}=0.253, y_{1(2)}=0.52$, and for period 2 are: $y_{2(1)}=0.029, y_{2(2)}=0.52$, $y_{2(3)}=0.991$. Note that the first period action is non-monotonic, but the average action is monotonic (lowest in $A$, highest in $C$ ).

An important feature of the constructed non-monotonic equilibrium is that it exists even though the only monotonic partition equilibrium in this (dynamic) game is a trivial babbling equilibrium. To see this, note that for bias $b=0.256$, the only equilibrium in the static game is babbling. Proposition 2 then implies that the only monotonic equilibrium in the dynamic game is babbling. At this equilibrium the decision maker takes the action $y^{B}=0.5$. The expert's and the decision maker's expected disutilities are respectively given by: -0.298 and -0.167 . Both the expert and the decision maker are strictly better-off at our non-monotonic equilibrium, where the disutilities are given by -0.275 for the expert and by -0.144 for the decision maker, compared to the most informative static equilibrium.

This example highlights how complex signalling can lead to better communication in a multistage game. By pooling together the best and the worst states at $t=1$, the expert is willing at $t=2$ to reveal whether the state is very good or very bad. It also has the following implication.

Proposition 3 There exist non-monotonic equilibria that are Pareto superior to all monotonic partition equilibria.

When preferences given by (1) and $b>0.25$, the only equilibrium in the static game is babbling, hence this Proposition follows immediately from the preceding discussion.

We now construct a non-monotonic equilibrium with even higher welfare properties. The key is to allow the expert to condition his future communication of the previously chosen action of the decision maker. We call such strategies trigger strategies. Now the expert uses the following signalling rule: if the state is in $[0,0.0642] \cup[0.9919,1]$ he tells the $D M$ at $t=1$ to choose 0.2615 and to choose 0.528 otherwise. The first action is above the myopic best response, which is $y_{1(1)}=$ $\frac{\underline{\theta}^{2}-\bar{\theta}^{2}+1}{2(\underline{\theta}-\bar{\theta}+1)}=0.14008$. If the $D M$ chooses the action recommended by the expert, then the expert at $t=2$ reveals whether the state is $[0,0.0642]$ or $[0.9919,1]$; otherwise, the expert threatens to babble. It is routine to check that this is an equilibrium. Moreover, at this equilibrium, the expert and the decision maker are even better off compared to our original non-monotonic equilibrium. Their disutilities are respectively given by: -0.2674 for the expert and by -0.1409 for the decision maker. The reason for the improvement, is that relying on a threat, we relax the best response constraint of the decision maker which increases the expected payoff of expert and, as a consequence, that of the decision maker (since their ex ante expected payoffs differ by $b^{2}$ ).

Our main result in the section that follows is that the dynamic set-up correlates the incentives 
of the expert and DM in such a way that full information revelation is possible. We present this next.

\section{Main Result: Full information revelation in dynamic cheap talk games}

One of the most stark results of the static strategic communication game is that there is no equilibrium with full information revelation. Although the state can take a continuum of values, the expert sends at most finitely many signals to the decision maker. That is, substantial amount of information is not transmitted. In this section, for our dynamic strategic communication model we construct an equilibrium that is fully revealing. The construction of the equilibrium relies on two tools: the use of separable groups, and the use of trigger strategies. These tools have no leverage in single-round communications, but are powerful in dynamic communications.

\subsection{Simple Case: Learning the Truth when the Expert is Myopic}

We first show how to construct a fully-revealing equilibrium when the expert is myopic. The example shows how we use separable groups of far away agents who mimic each other but not the nearby types. With a myopic expert, there is no need to use trigger strategies.

There are two essential ingredients of this example. First, the set of types that choose the same message at $t=1$ are sufficiently far apart so they can be separated at $t=2$. That is, each message is chosen by a separable group of types. Second, the average of types in each group is constant. The first period action is then flat. Irrespective of the state, all experts induce the same action. This implies that the expert does not care which group he joins (since a myopic expert cares only about the 1st-period action, which is constant across groups).

Example 2 Fully revealing equilibrium with impatient experts $\left(\delta_{E}=0\right)$.

Consider the uniform quadratic loss case as in (1) when the bias is $b<\frac{1}{16}$ and $T=2$. The expert is impatient $\left(\delta_{E}=0\right)$. The construction works for any discount factor for the decision maker. In period 1 , the expert for states $\left\{\frac{1}{8}-\varepsilon ; \frac{3}{8}+\varepsilon, \frac{4}{8}+\varepsilon, 1-\varepsilon\right\}$ sends message $m_{\varepsilon}$, for $\varepsilon \in\left[0, \frac{1}{8}\right]$. For

states $\left\{\frac{1}{8}+\tilde{\varepsilon}, \frac{7}{8}-\tilde{\varepsilon}\right\}$, the expert sends a message $m_{\tilde{\varepsilon}}$ for $\tilde{\varepsilon} \in\left(0, \frac{1}{4}\right)$. That is, we have two types of separable groups: indexed by $\varepsilon$ and $\tilde{\varepsilon}$. Given this signalling rule, the best response of the decision maker in period 1 is to choose:

$$
\begin{aligned}
& y\left(m_{\varepsilon}\right)=\frac{\frac{1}{8}-\varepsilon+\frac{3}{8}+\varepsilon+\frac{4}{8}+\varepsilon+1-\varepsilon}{4}=0.5 \text { for all } \varepsilon \in\left[0, \frac{1}{8}\right], \\
& y\left(m_{\tilde{\varepsilon}}\right)=\frac{\frac{1}{8}+\tilde{\varepsilon}+\frac{7}{8}-\tilde{\varepsilon}}{2}=0.5 \text { for all } \tilde{\varepsilon} \in\left(0, \frac{1}{4}\right) .
\end{aligned}
$$


In period 2, the expert tells the truth, inducing an action equal to the state. After any out-ofequilibrium initial message, the decision maker assigns equal probability to all states, leading to action $y^{\text {out }}=0.5$. After any out-of-equilibrium second-period message, the decision maker assigns probability 1 to state 0 , and accordingly chooses $y^{\text {out }}=0$.

If the expert is impatient $\left(\delta_{E}=0\right)$, then for any $b<\frac{1}{16}$ we argue that this is an equilibrium.

First, notice that all messages (even out-of-equilibrium ones) induce the same action in period 1. Hence, the expert is indifferent between all possible first-period actions, and therefore indifferent between all possible first-period messages if he puts zero weight on the future. So, in particular, a myopic expert will find it optimal to send the "right" message, following the strategy outlined above. Now consider, for example, the history following an initial message $m_{\varepsilon}$. The DM's posterior beliefs assign probability $\frac{1}{4}$ to each of the following types: $\frac{1}{8}-\varepsilon ; \frac{3}{8}+\varepsilon, \frac{4}{8}+\varepsilon$ and $1-\varepsilon$. If at stage 2 each of these types sends a different message, namely $m_{2(k)}$, where $k \in\left\{\frac{1}{8}-\varepsilon ; \frac{3}{8}+\varepsilon, \frac{4}{8}+\varepsilon, 1-\varepsilon\right\}$, then after each of these messages, the decision maker chooses $y\left(m_{2(k)}\right)=k$. To see that at $t=2$ the expert has no incentive to deviate, we consider, for instance, type $\theta=\frac{3}{8}+\varepsilon$ and show that he does not have incentive to mimic type $\theta+\frac{1}{8}:-(\theta-\theta-b)^{2}>-\left(\theta+\frac{1}{8}-\theta-b\right)^{2}$, or $\left(\frac{1}{8}-b-b\right) \frac{1}{8}>0$, which is satisfied if $b<\frac{1}{16}$. A similar argument holds for other types. Additionally, any out-of equilibrium message at $t=2$, leads to $y^{\text {out }}=0$ which is always worse than $y_{2}(\theta)=\theta$ since $-b^{2} \geq-(b+\theta)^{2}$ for all $\theta \in[0,1]$. The situation after a message $m_{\tilde{\varepsilon}}$ is analogous.

This construction does not apply when the expert is patient, because it does not provide the expert with incentives to join the "right" separable group as we discuss below. We now turn to the case of a patient expert.

\subsection{Full Information Revelation with a Patient Expert}

The main contribution of the paper is to construct a fully revealing equilibrium when the expert is patient.

Theorem 1 Suppose that $\delta_{E}=\delta_{D M}=1$ and that the preferences of the expert and of the decision maker are given by (1). For any bias $b \leq \frac{1}{61}$, there is an infinite set of priors $F$, and a horizon $T^{*}$, for which a fully revealing equilibrium exists whenever $T \geq T^{*}$.

We provide a sketch of the proof and leave the details to the appendix.

There are two key constructs in the proof.

The first is the use of the separable groups, as in the case of the myopic expert above. Rather than having intervals of types pool together, we construct pairs of far-away types ("partners") who pool together in the initial periods. The advantage is that once the expert joins one of these separable groups, revealing the two possible true states to the DM, we no longer need to worry 
about him mimicking nearby types: his only options are to tell the truth, or to mimic his partner. Of course, an important part of the proof is to ensure that each expert type wants to join the "right" separable group. For a myopic expert, this is straightforward: if the expert cares only about the first-period action, then to make him join the right group, it is sufficient that the first-period action be constant across groups. (In fact, the myopic expert result relied only on separable groups, without the need for trigger strategies: we were able to group types such that the (constant) action recommended by each group was equal to the average type within the group, i.e. so that it coincided with the DM's myopically optimal choice). For a patient expert, the construction is significantly more involved, as it must take dynamic incentives into account. In particular, the actions induced in the initial stages cannot be flat: if this were the case, then an expert who cares about the future would simply join whichever separable group leads to the best future action. Hence, for a patient expert, we need to construct initial action functions which provide appropriate incentives: if type $\theta$ knows that some type $\theta^{\prime}$ will get a more favorable action in the revelation phase, then type $\theta$ 's group must induce an initial action which is more favorable to type $\theta$ than that induced by $\left(\theta^{\prime}\right)$ 's group.

The second key part of the construction is the use of the trigger strategies. The need for the trigger strategies is closely related to the construction of the separable groups. In the revelation stage, the decision maker gets rewarded as the experts reveal the truth. However, this revelation is obviously costly for the expert, and, as outlined in the previous paragraph, requires initial actions which provide appropriate rewards. These expert-incentivizing actions turn out to be incompatible with the myopically optimal actions from the DM's perspective. Therefore, to make the DM cooperate with the equilibrium, we need to use trigger strategies. The expert threatens that if the DM rejects his early advice, the expert will stop revealing information. If the DM follows the expert's advice, the DM will be rewarded with eventual full revelation of the state.

Before we proceed with the sketch, it is useful to simplify notation and work with a scaled type space by dividing all actions and types by $b$. When we say that "type $\theta \in\left[0, \frac{1}{b}\right]$ recommends $u(\theta)$ in period 1 , for disutility $(u(\theta)-\theta-1)^{2}$ ", we mean that (in the unscaled type space) type $\theta b$ recommends action $u(\theta) b$, for disutility $(u(\theta) b-\theta b-b)^{2}=b^{2}(u(\theta)-\theta-1)^{2}$.

We first partition the scaled type space $\left[0, \frac{1}{b}\right]$ into four intervals, with endpoints $\left[0, \theta_{1}, \theta_{2}, \theta_{3}, \frac{1}{b}\right]$. The separable groups are as follows: at time $t=0$, each type $\theta \in\left[0, \theta_{1}\right]$ pools with a partner $g(\theta) \in\left[\theta_{2}, \theta_{3}\right]$ to send an sequence of recommendations $\left(u_{1}(\theta), u_{2}(\theta)\right)$, and then reveal the truth at time $t=2$ iff the DM followed both initial recommendations. Each type $\theta \in\left[\theta_{1}, \theta_{2}\right]$ initially pools with a partner $h(\theta) \in\left[\theta_{3}, \theta_{4}\right]$ to recommend a sequence $\left(v_{1}(\theta), v_{2}(\theta)\right)$, then revealing the truth at time $T-\tau$ (iff the expert followed their advice). ${ }^{8}$ For the purpose of this outline, take the endpoints

\footnotetext{
${ }^{8}$ Note that $u_{1}, u_{2}, v_{1}, v_{2}$ are functions of $\theta$, and that in our construction, the expert's messages ("recommendations") are equal to the actions that he wants the DM to take.
} 
$\theta_{1}, \theta_{2}, \theta_{3}$ as given, along with the partner functions $g:\left[0, \theta_{1}\right] \rightarrow\left[\theta_{2}, \theta_{3}\right] ; h:\left[\theta_{1}, \theta_{2}\right] \rightarrow\left[\theta_{3}, \theta_{4}\right]$, and recommendation functions $u_{1}, u_{2}, v_{1}, v_{2}$. In the appendix, we derive the parameters and functions that work, and provide the full details of how to construct fully revealing equilibria.

We now describe the strategy for the expert and for the decision maker. For notational purposes it is useful to further subdivide the expert types into three groups: $I, I I$, and $I I I$.

At time $t=0$, there are then three groups of experts. Group $I$ consists of types $\theta^{I} \in\left[\theta_{1}, \theta_{2}\right]$ with their partners $h\left(\theta^{I}\right) \in\left[\theta_{3}, \frac{1}{b}\right]$. Group $I I$ consists of all types $\theta^{I I} \in\left[0, \theta_{1}\right]$ whose initial recommendation coincides with that of a Group I pair, together with their partners $g\left(\theta^{I I}\right) \in\left[\theta_{2}, \theta_{3}\right]$. Group $I I I$ consists of all remaining types $\theta^{I I I} \in\left[0, \theta_{1}\right]$ and their partners $g\left(\theta^{I I I}\right) \in\left[\theta_{2}, \theta_{3}\right]$. In other words, we divided the types in intervals $\left[0, \theta_{1}\right] \cup\left[\theta_{2}, \theta_{3}\right]$ into two groups, $I I$ and $I I I$, according to whether or not their initial messages coincide with that of a group $I$ pair.

The timeline of the expert's advice is as follows:

\begin{tabular}{|c|c|c|c|c|}
\hline$\downarrow$ & $\downarrow$ & $\downarrow$ & $\downarrow$ & $\downarrow$ \\
\hline Phase $1(t=0)$ & Phase $2 \quad\left(t=2 \alpha_{0}\right)$ & Phase $3\left(t=2 \alpha_{a}\right)$ & Phase $4(t=2)$ & Phase $5(T-\tau)$ \\
\hline $\begin{array}{l}\text { Group } I: v_{1} \\
\text { Groups } I I, I I I: u_{1}\end{array}$ & $\begin{array}{l}\text { Group } I: \\
\text { switch to } v_{2}\end{array}$ & $\begin{array}{c}\text { Groups } I I, I I I: \\
\text { switch to } u_{2} \\
\text { Figure 1: Timeline }\end{array}$ & $\begin{array}{l}\text { Groups } I I, I I I: \\
\text { reveal the truth }\end{array}$ & $\begin{array}{l}\text { Group } I: \\
\text { reveal the truth }\end{array}$ \\
\hline
\end{tabular}

where $0<\alpha_{0} \leq \alpha_{a}<1$ are specified in the appendix (section D.2.1).

In words: in the initial phase, types in Group $I$ recommend $v_{1}: v_{1}\left(\theta^{I}\right)=v_{1}\left(h\left(\theta^{I}\right)\right)$, while types in Groups $I I$ and $I I I$ recommend the action $u_{1}: u_{1}\left(\theta^{I I}\right)=u_{1}\left(g\left(\theta^{I I}\right)\right)$. Importantly, the recommendations for the types in Group $I$ and Group $I I$ coincide (while Group III recommendations do not coincide with those of any Group $I$ pair): for every $\theta^{I}$ in Group I, there exists $\theta^{I I}$ in Group $I I$ with $v_{1}\left(\theta^{I}\right)=u_{1}\left(\theta^{I I}\right)$. This is why, for ease of exposition, we have a subdivision into Groups $I I$ and $I I I$; upon receiving a recommendation $v_{1}\left(\theta^{I}\right)=u_{1}\left(\theta^{I I}\right)$, the DM believes that it could have come from any of the types in $\left\{\theta^{I}, h\left(\theta^{I}\right), \theta^{I I}, g\left(\theta^{I I}\right)\right\}$ (see footnote 16 for why this is needed). At time $t=2 \alpha_{0}$, group $I$ pairs $\left\{\theta^{I}, h\left(\theta^{I}\right)\right\}$ change their recommendation to $v_{2}\left(\theta^{I}\right)=v_{2}\left(h\left(\theta^{I}\right)\right)$, while Groups II and III continue to follow the recommendation function $u_{1}$ (that is, they do not yet change their advice). Thus, at this stage, the DM learns whether he is facing a Group I, II, or III pair. At time $t=2 \alpha_{a} \geq 2 \alpha_{0}$, group $I I$ and $I I I$ pairs switch to the recommendation function $u_{2}$, where $\alpha_{a}$ may depend on the specific pair. ${ }^{9}$ Group $I$ continues to follow the recommendation function $v_{2}$, revealing no further information at this stage. At time $t=2$, group $I I$ and $I I I$ pairs separate: each type $\theta^{I I}$ or $\theta^{I I I}$ in $\left[0, \theta_{1}\right]$ sends a message equal to his type (thus revealing the truth for the final $T-2$ periods), and similarly their partners $g\left(\theta^{I I}\right), g\left(\theta^{I I I}\right)$ send messages equal to their

\footnotetext{
${ }^{9}$ See Lemma D7.1 in the appendix: we describe group II and III pairs parametrically as functions $\{x(a), g(a)\}$ of a variable $a$, along with their recommendation functions $u_{1}, u_{2}$, and specify a time $t=2 \alpha_{a} \in\left[2 \alpha_{0}, T-\tau\right]$ at which the pair switches from recommendation $u_{1}(a)$ to $u_{2}(a)$.
} 
own types. At time $T-\tau>2$, Group I pairs $\left\{\theta^{I}, h\left(\theta^{I}\right)\right\}$ separate, with type $\theta^{I}$ recommending action $\theta^{I}$ and type $h\left(\theta^{I}\right)$ recommending $h\left(\theta^{I}\right)$ for the final $\tau$ periods. It should be noted that the times at which the DM is instructed to change his action $\left(2 \alpha_{0}, 2 \alpha_{a}, T-\tau\right)$ are not necessarily integers in our construction. In a continuous time setting, this clearly poses no problem; if there are integer constraints, it is straightforward to "scale up" all the time parameters (see footnote 18 in Appendix D).

The DM's strategy is to follow all on-path recommendations. An off-path recommendation at time $t=0$ is treated as a mistake coming from the pair $\{0, g(0)\}$, and subsequent off-path recommendations are simply ignored as errors (full details in the appendix, section D.2).

To summarize: In the initial phase, separable groups are formed. Each expert type sends a recommendation sequence of the form $(\underbrace{v_{1}\left(\theta^{I}\right)}_{2 \alpha_{0} \text { periods }}, \underbrace{v_{2}\left(\theta^{I}\right)}_{T-\tau-2 \alpha_{0} \text { periods }})$ or $(\underbrace{u_{1}\left(\theta^{i}\right)}_{2 \alpha_{a} \text { periods }}, \underbrace{u_{2}\left(\theta^{i}\right)}_{2\left(1-\alpha_{a}\right) \text { periods }})$, with $i \in\{I I, I I I\}$, and such that for all $\theta^{I} \in\left[\theta_{1}, \theta_{2}\right]$, there exists $\theta^{I I} \in\left[0, \theta_{1}\right]$ with $v_{1}\left(\theta^{I}\right)=u_{1}\left(\theta^{I I}\right)$. During these phases, the decision-maker is able to infer the separable group containing the expert's true type, but, rather than choosing the corresponding myopically optimal action, he chooses the actions $u_{1}, u_{2}, v_{1}, v_{2}$ recommended by the expert. These action functions are constructed to provide the expert with incentives to join the right separable group at time 0 . The final phases are the revelation phases: The separable groups themselves separate, revealing the exact truth to the decision-maker, provided that he has followed all of the expert's previous advice; any deviation results in babbling by the expert during the revelation phase.

Finally, we briefly comment on the construction of the functions $\left(u_{1}, u_{2}\right)$ and $\left(v_{1}, v_{2}\right)$, and the corresponding partner functions $g, h$ (and endpoints $\theta_{1}, \theta_{2}, \theta_{3}$ ), which are given parametrically in the appendix (section D.1). For the expert, three sets of constraints must be satisfied:

Expert Local IC: The first set of constraints can be thought of as local incentive compatibility constraints-that is, those applying within each type $\theta$ 's interval $\left[\theta_{i}, \theta_{i+1}\right]$. These (dynamic) incentive compatibility constraints ensure that, say, the agent $\theta \in\left[0, \theta_{1}\right]$ prefers to induce actions $u_{1}(\theta)$ (for $2 \alpha_{a}$ periods), $u_{2}(\theta)$ (for $2\left(1-\alpha_{a}\right)$ periods), and then reveal his type $\theta$ for the final $T-2$ periods, rather than e.g. following the sequence $\left(u_{1}\left(\theta^{\prime}\right), u_{2}\left(\theta^{\prime}\right), \theta^{\prime}\right)$ prescribed for some other type $\theta^{\prime}$ in the same interval $\left[0, \theta_{1}\right]$ (and analogously within each of the other three intervals). For types $\theta \in\left[0, \theta_{1}\right]$, this boils down to a requirement that $u_{1}, u_{2}$ satisfy the following differential equation,

$$
2 \alpha_{a} u_{1}^{\prime}(\theta)\left(u_{1}(\theta)-\theta-1\right)+2\left(1-\alpha_{a}\right) u_{2}^{\prime}(\theta)\left(u_{2}(\theta)-\theta-1\right)=T-2
$$

and that the "average" action, $2 \alpha_{a} u_{1}(\theta)+2\left(1-\alpha_{a}\right) u_{2}(\theta)+(T-2) \theta$, be weakly increasing in $\theta$. Note that a longer revelation phase (that is, an increase in the RHS term $(T-2)$ ) requires a correspondingly larger distortion in the action functions $u_{1}, u_{2}$ : if the expert anticipates a lengthy 
phase in which the DM's action will match the true state (whereas the expert's bliss point is to the right of the truth), then it becomes more difficult in the initial phase to provide him with incentives not to mimic the advice of types to his right.

Expert Global IC: The next set of constraints for the expert can be thought of as "global" incentive compatibility constraints, ensuring that no expert type wishes to mimic any type in any other interval. In the appendix, we show that this boils down to two additional requirements: each endpoint type $\theta_{1}, \theta_{2}, \theta_{3}$ must be indifferent between the two equilibrium sequences prescribed for his type (for example, type $\theta_{1}$ must be indifferent between sequences $(\underbrace{u_{1}\left(\theta_{1}\right)}_{2 \alpha_{a}}, \underbrace{u_{2}\left(\theta_{1}\right)}_{2\left(1-\alpha_{a}\right)}, \underbrace{\theta_{1}}_{T-2})$ and $(\underbrace{v_{1}\left(\theta_{1}\right)}_{2 \alpha_{0}}, \underbrace{u_{2}\left(\theta_{1}\right)}_{T-\tau-2 \alpha_{0}}, \underbrace{\theta_{1}}_{\tau})$, and the "average" action must be either continuous or jump up at each endpoint.

Expert Separation: The final constraint requires that each pair of types indeed be "separable", that is, sufficiently far apart that each type would rather tell the truth than mimic his partner. In our rescaled type space, this requires choosing partner functions $g$, $h$ satisfying $|g(\theta)-\theta| \geq 2$ and $|h(\theta)-\theta| \geq 2$, which we do in the appendix (Proposition D2). It turns out to be very tricky to satisfy the global incentive compatibility constraints together with the local constraints: it in fact requires a minimum of two distinct actions prior to the revelation phase (this is why e.g. Group III pairs must change their recommendation from $u_{1}$ to $u_{2}$ at time $2 \alpha_{a}$, even though doing so reveals no further information), and that the type space be partitioned into a minimum of four intervals. Moreover, for any partition into four intervals, there is in fact only one partner function $g:\left[0, \theta_{1}\right] \rightarrow\left[\theta_{2}, \theta_{3}\right]$ that works, and we believe that there is no partition which would allow for expert-incentivizing action functions which are myopically optimal from the DM's perspective. This is why our construction relies on trigger strategies: the expert only reveals the truth if the DM follows all of his advice.

Incentivizing the Decision-maker: For the decision-maker, we need to make sure that the benefit to learning the exact state (rather than just the separable group that contains it) is large enough to compensate him for the cost of following the expert's initial recommendations, rather than deviating to the myopically optimal actions. This is what limits the priors for which our construction works, and imposes the upper bound $b \cong \frac{1}{61}$ on the bias. The construction works for the expert $\forall b<\frac{1}{16}$.

Discussion: The true state is revealed at either time 2 or time $T-\tau$, where in the appendix we require $T-\tau \leq 4$. Thus, the decision-maker chooses his best possible action, equal to the true state, in all but the first few periods. It is tempting to conclude that a long horizon means an 
equilibrium approaching the first-best, but unfortunately this is not true when the decision-maker and expert are equally patient. As explained after equation (4), a long horizon also makes it difficult to incentivize the expert, requiring a proportionally larger distortion in the initial recommendation functions, and thereby imposing a proportionally larger cost to the DM (from having to follow such bad early advice in order to learn the truth). We do, however, conjecture that if the decisionmaker is more patient than the expert, our fully revealing equilibrium would have attractive welfare properties, and work for a large range of prior beliefs: If the expert doesn't care much about the future, it becomes easy to incentivize him to join the right separable group, which, in turn, implies little need to distort the initial recommendations, and therefore little cost to the DM from following bad advice in the first couple of periods. The benefit to following this advice- knowing the exact optimal action in all but the first few periods-will then outweigh this cost for a patient DM. (Section 5.1 illustrated this in the extreme case $\delta_{E}=0$, where the DM learned the exact truth with no distortion in the expert's initial advice). Also, if we look at situations where the decision maker cares only about the ultimate decision, it is easy to see that our construction works for any prior (for all $b<\frac{1}{16}$ ), and yields the best possible outcome for the DM.

\section{Concluding Remarks}

This paper shows that dynamic strategic communication differs from its static counterpart. Our most striking result is that fully revealing equilibria exist. We also present a series of examples that show how the canonical results of the static strategic communication may not hold when communication is repeated.

The main novel ingredient of our model is that there are many (finite) rounds of communication, and after each round an action is chosen. The dynamic considerations of the expert allow us to group together types that are far apart, forming "separable" groups which is the key ingredient behind the construction of fully revealing equilibria and of non-monotonic equilibria. The dynamic setup of our model also allows for future communication to be conditioned on past actions (trigger strategies). We show how information revelation can be facilitated through this channel.

Our analysis highlights the nature of incentive compatibility in dynamic incentive settings. One important characteristic is that dynamic incentive constraints can bind for a disconnected set of types. Moreover, the fact that posteriors are endogenous, implies that earlier rounds in the game can be used to induce posteriors that eventually allow the decision maker to learn the truth. At those nodes in the game the information rents of the expert disappear.

The forces that we identify can be present in other dynamic environments where there is asymmetric information and players behave sequentially rationally. Think, for example, a dynamic contracting environment where there is limited commitment about the future, or, more generally, a 
dynamic mechanism design problem, or some other dynamic game where one imposes the requirement of sequential rationality. In those models as well, past behavior setups the stage for future behavior because posteriors are endogenous. And, in contrast to the vast majority of the recent literature on dynamic mechanisms design, ${ }^{10}$ one needs to worry about global incentive compatibility despite the fact that stage payoffs satisfy the single-crossing property.

Lastly, given the important insights that the theory of static strategic communication has lead to for many aspects of economics and of political science, we hope and expect that the novel aspects of strategic communication that our analysis emphasizes will help shed new light to many interesting phenomena. They could certainly be useful in thinking about the design of optimal communication protocols and even about the design of questionnaires.

\footnotetext{
${ }^{10}$ In recent years motivated by the large number and the importance of applications there has been substantial work on dynamic mechanism design. See, for example, the survey of Bergemann and Said (2010), and the references therein, or Athey and Segal (2007) who characterize a dynamic version of the VCG mechanism that is efficient and balances the budget.
} 
APPENDIX

\section{A Proof of Proposition 2}

When we restrict attention to monotonic partition equilibria there will be some point in the game where the last subdivision of an interval into smaller partitions occurs. This can either happen at the last period, or at some period before last. Assume without loss, that one interval is partitioned into two inducing actions $y_{1}\left(m_{1}\right)$ and $y_{2}\left(m_{2}\right)$. If it happens in the last period $T$ of the game, then the indifference condition of the expert type - call that type $\hat{\theta}$ - that is indifferent between $y_{1}$ and $y_{2}$ is the static one. But then, if a subinterval of the original state space can divided into two, it cannot be the case that all static equilibria are equivalent babbling. This follows by Corollary 1 of Crawford and Sobel (1982)).

Now, suppose that the last subdivision happens at a period before the last. Say at period $\hat{T}$. Given that no further subdivision occurs after $\hat{T}$, when the expert sends a message that induces $y_{1}$ (resp. $y_{2}$ ) at $\hat{T}$ the same action is induced for the remaining number of periods $T-\hat{T}$. Then the indifference condition for type $\hat{\theta}$ expert is

$$
(T-\hat{T}) u^{E}\left(y_{1}, \hat{\theta}\right) \geq(T-\hat{T}) u^{E}\left(y_{2}, \hat{\theta}\right),
$$

which is equivalent to static. But then, as before, this subdivision at $\hat{T}$ is not possible given that all static equilibria are equivalent babbling.

Observe that all the arguments in this proof go through even if we allow for trigger strategies. This is because at the point where the last subdivision occurs, it is impossible to incentivize the decision maker to do something worse than his myopic best response. To see this, note at that point, either the game ends - when $\hat{T}=T$, so there is no time to reward the decision maker for choosing a suboptimal action at $T$ or $\hat{T}<T$. Suppose that $\hat{T}<T$, and assume without loss, that one interval is partitioned into two inducing actions $\tilde{y}_{1}\left(m_{1}\right)$ and $\tilde{y}_{2}\left(m_{2}\right)$ and suppose that $\tilde{y}_{1}$ generates a payoff for the DM different from the myopic best response $y_{1}$, then in order for the DM to follow $\tilde{y}_{1}$ it must hold

$$
(T-\hat{T}) E_{\theta}\left[u^{D M}\left(\tilde{y}_{1}, \theta\right) \mid m_{1}\right]>(T-\hat{T}) E_{\theta}\left[u^{D M}\left(y_{1}, \theta\right) \mid m_{1}\right]
$$

which contradicts the fact that $y_{1}$ is the myopic best response given $m_{1}$. Hence, the DM cannot choose actions that give him a payoff different from the payoff at the myopic best response. This together with the strict concavity of $u^{D M}$ imply that $y_{1}=\tilde{y}_{1}$.

\section{B Monotonic partition equilibria with more partitions}

Suppose that $\delta_{E}=\delta_{D M}=1$, types are uniformly distributed on $[0,1]$ and preferences satisfy (1). Suppose that bias $b=\frac{1}{12}$. Using standard arguments, one can establish that game has only two equilibria, ${ }^{11}$ a babbling equilibrium and an equilibrium with two partitions, where the space is divided in two sub-intervals: $\left[0, \frac{1}{3}\right]$ and $\left[\frac{1}{3}, 1\right]$ inducing two actions $\frac{1}{6}$ and $\frac{4}{6}$. Now we show that when

${ }^{11}$ The largest number of subintervals that the type space can be divided into is the largest integer that satisfies

$$
-2 b p^{2}+2 b p+1>0
$$

whose solution is

$$
\left\langle-\frac{1}{2}+\frac{1}{2} \sqrt{1+\frac{2}{b}}\right\rangle,
$$

and where $\langle x\rangle$ denotes the smallest integer greater than or equal to $x$. 
$T=2$, there exists a monotonic partition equilibrium where in the end the state space is divided in three sub-intervals.

We look for an equilibrium with the following signaling rule:

types in $\left[0, \theta_{1}\right]$ send message sequence $A=\left(m_{1(1)}, m_{2(1)}\right)$,

types in $\left[\theta_{1}, \theta_{2}\right]$ send message sequence $B=\left(m_{1(2)}, m_{2(2)}\right)$,

types in $\left[\theta_{2}, 1\right]$ send message sequence $C=\left(m_{1(2)}, m_{2(3)}\right)$.

With this signaling rule in the first period the interval $[0,1]$ is partitioned into $\left[0, \theta_{1}\right]$ and $\left[\theta_{1}, 1\right]$. At stage $2,\left[\theta_{1}, 1\right]$ is partitioned further into $\left[\theta_{1}, \theta_{2}\right]$ and $\left[\theta_{2}, 1\right]$. We can express $\theta_{2}$ in terms of $\theta_{1}$ as follows:

$$
\theta_{2}=\left(\frac{1-\theta_{1}}{2}-\frac{4}{12}\right)+\frac{2}{12}+\theta_{1}=\frac{1}{2} \theta_{1}+\frac{1}{3} .
$$

The second period actions induced are $y_{2(1)}=\frac{\theta_{1}}{2}, y_{2(2)}=\frac{3}{4} \theta_{1}+\frac{1}{6}$ and $y_{2(3)}=\frac{1}{4} \theta_{1}+\frac{2}{3}$, whereas the first period actions induced are $y_{1(1)}=\frac{\theta_{1}}{2}$ and $y_{1(2)}=\frac{1+\theta_{1}}{2}$.

After any out-of- equilibrium message the decision maker assigns probability one to the state belonging in $\left[0, \theta_{1}\right]$ inducing $y^{\text {out }}=\frac{\theta_{1}}{2}$. With these out-of equilibrium beliefs it is immediate to see that no type has an incentive to send an out-of-equilibrium message.

At an equilibrium $\theta_{1}$ must satisfy the following indifference condition:

$$
-\left(\frac{1+\theta_{1}}{2}-\theta_{1}-\frac{1}{12}\right)^{2}-\left(\frac{\frac{1}{2} \theta_{1}+\frac{1}{3}+\theta_{1}}{2}-\theta_{1}-\frac{1}{12}\right)^{2}=-2\left(\frac{\theta_{1}}{2}-\theta_{1}-\frac{1}{12}\right)^{2},
$$

which with the help of (7) gives us 3 partitions with cutoffs $\theta_{1}=0.25$ and in turn $\theta_{2}=0.45833$. Using this value of $\theta_{1}$ we get that: $y_{1(1)}=y_{2(1)}=0.125, y_{1(2)}=0.625, y_{2(2)}=0.35417$ and $y_{2(3)}=0.72917$.

In constructing this strategy profile we have imposed that type $\theta_{2}$ is indifferent between inducing action $y_{2(2)}$ and $y_{2(3)}$ at $t=2$ and that type $\theta_{1}$ is indifferent between inducing action sequence $y_{1(1)}$ and $y_{2(1)}$ and $y_{1(2)}$ and $y_{2(2)}$. Now we want to verify that these conditions are sufficient for global incentive compatibility. At $t=2$ the game is isomorphic to the static one, where the fact that $\theta_{2}$ is indifferent between $y_{2(2)}$ and $y_{2(3)}$ implies that all types above $\theta_{2}$ prefer $y_{2(3)}$ and all types below $\theta_{2}$ prefer $y_{2(2)}$. To verify that types below $\theta_{1}$ prefer message sequence $A$ inducing $y_{1(1)}$ and $y_{2(1)}$, and types above $\theta_{1}$ prefer message sequence $B$ inducing $y_{1(2)}$ and $y_{2(2)}$, we plot the difference $U(A, \theta)-U(B, \theta)$ and show that it is positive for all $\theta<\theta_{1}$ and negative for $\theta>\theta_{1}$ :

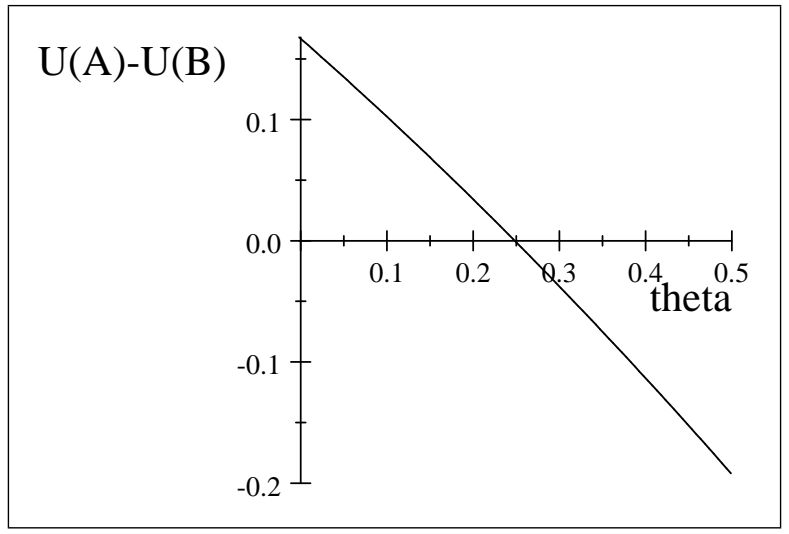

At the dynamic equilibrium we constructed here the expert's welfare is -0.065 and the decision maker's welfare is given by -0.0517 , which are both higher than the welfare at the equilibrium where the most informative static equilibrium is played in the first period, and babbling in the second, which are respectively -0.069 and -0.055 . 


\section{Pareto comparisons of dynamic cheap talk equilibria}

The following examples demonstrate that equilibria with more partitions can be Pareto inferior to the equilibria with fewer partitions

Take $\delta_{E}=\delta_{D M}=1$ and $b=0.08$, and consider the most informative static partition equilibrium where the number of partitions is $p=3$. At this equilibrium the state space is divided into [0,0.013], $[0.013,0.347]$ and $[0.347,1]$. The corresponding optimal actions of the decision maker are given by

$$
y_{1}=0.0067 \quad y_{2}=0.18 \quad y_{3}=0.673
$$

from which we can calculate the ex-ante expected utility levels for the expert -0.032 and for the decision maker -0.0263 . Then, at the equilibrium of the dynamic game where the most informative static equilibrium is played at $t=1$ and babbling thereafter, the total expected utility is -0.065 for the expert, and -0.053 for the decision maker.

We now construct a dynamic equilibrium where the type space is subdivided into more subintervals, but both players' ex-ante expected payoffs are lower. We look for an equilibrium with the following signaling rule:

$$
\begin{aligned}
& \text { types in }\left[0, \theta_{1}\right] \text { send message sequence }\left(m_{1(1)}, m_{2(1)}\right) \\
& \text { types in }\left[\theta_{1}, \theta_{2}\right] \text { send message sequence }\left(m_{1(2)}, m_{2(2)}\right) \\
& \text { types in }\left[\theta_{2}, \theta_{3}\right] \text { send message sequence }\left(m_{1(2)}, m_{2(3)}\right) \\
& \text { types in }\left[\theta_{3}, 1\right] \text { send message sequence }\left(m_{1(3)}, m_{2(4)}\right) .
\end{aligned}
$$

So types are partitioned into four intervals in stage 2 , but in stage 1 , the types in $\left[\theta_{1}, \theta_{2}\right]$ and $\left[\theta_{2}, \theta_{3}\right]$ pool together to send the same message $m_{1(2)}$. Since the signaling rule does not depend on the decision maker's action at stage 1 , the decision maker will choose the following myopically optimal actions:

$$
\begin{aligned}
& y_{1(1)}=y_{2(1)}=\frac{\theta_{1}}{2} \\
& y_{1(2)}=\frac{\theta_{1}+\theta_{3}}{2}, y_{2(2)}=\frac{\theta_{1}+\theta_{2}}{2}, y_{2(3)}=\frac{\theta_{2}+\theta_{3}}{2}, \\
& y_{1(3)}=y_{2(4)}=\frac{1+\theta_{3}}{2} .
\end{aligned}
$$

After any out-of-equilibrium message the decision maker assigns probability one to the state belonging in $\left[0, \theta_{1}\right]$ inducing $y^{\text {out }}=\frac{\theta_{1}}{2}$. With these out-of-equilibrium beliefs it is immediate to see that no type has an incentive to deviate.

In equilibrium, type $\theta_{1}$ is indifferent between action sequences $\left\{y_{1(1)}, y_{2(1)}\right\}$ and $\left\{y_{1(2)}, y_{2(2)}\right\}$, type $\theta_{2}$ is indifferent between 2nd-period actions $y_{2(2)}$ and $y_{2(3)}$, and type $\theta_{3}$ is indifferent between action sequences $\left\{y_{1(2)}, y_{2(3)}\right\}$ and $\left\{y_{1(3)}, y_{2(4)}\right\}$. Therefore, equilibrium cutoffs are the solution to 
the following system of equations: ${ }^{12}$

$$
\begin{aligned}
2\left(\frac{\theta_{1}}{2}-\theta_{1}-b\right)^{2}- & \left(\frac{\theta_{1}+\theta_{3}}{2}-b-\theta_{1}\right)^{2}-\left(\frac{\theta_{1}+\theta_{2}}{2}-b-\theta_{1}\right)^{2}=0 \\
& \left(\frac{\theta_{1}+\theta_{2}}{2}-b-\theta_{2}\right)^{2}-\left(\frac{\theta_{2}+\theta_{3}}{2}-b-\theta_{2}\right)^{2}=0 \\
2\left(\frac{1+\theta_{3}}{2}-b-\theta_{3}\right)^{2}- & \left(\frac{\theta_{1}+\theta_{3}}{2}-b-\theta_{3}\right)^{2}-\left(\frac{\theta_{2}+\theta_{3}}{2}-b-\theta_{3}\right)^{2}=0 .
\end{aligned}
$$

At $b=0.08$, the only solution that gives numbers in $[0,1]$ is $\theta_{1}=0.0056, \theta_{2}=0.015, \theta_{3}=0.345$, and the actions induced for $t=1$ and for $t=2$ are respectively given by $y_{1(1)}=y_{2(1)}=0.00278$, $y_{1(2)}=0.175, y_{2(2)}=0.0105, y_{2(3)}=0.18$ and $y_{1(3)}=y_{2(4)}=0.673$. This implies the following total ex-ante expected utility for the expert -0.066 , which is lower than $2(-0.033)=-0.0656$. The utility for the decision-maker is -0.053 which is lower than $2(-0.026)=0.052$.

This example illustrates that although the interval is divided into more subintervals here, both players strictly worse off compared to the one where the most informative static equilibrium is played in the first period and babbling thereafter. The feature that less partitions lead to higher ex-ante welfare for both players also appears in example 1 of Blume, Board and Kawamura (2007).

\section{Proof of Theorem 1}

We will prove by construction that a fully revealing equilibrium exists. We first choose the endpoints $\theta_{1}, \theta_{2}, \theta_{3}$ described in the proof outline: for any bias $b<\frac{1}{61}$, define $a_{\gamma}<0$ by

$$
\left(a_{\gamma}-2+2 e^{-a_{\gamma}}\right) e^{2}-a_{\gamma}=\frac{1}{b}
$$

and then set

$$
\theta_{3}=\frac{1}{b}+a_{\gamma}, \theta_{2}=\theta_{3}-2, \theta_{1}=\theta_{2}-\theta_{3} e^{-2}
$$

It will be convenient to describe types parametrically, via functions $x:[-2,0] \rightarrow\left[0, \theta_{1}\right], g$ : $[-2,0] \rightarrow\left[\theta_{2}, \theta_{3}\right], z:\left[a_{\gamma}, 0\right] \rightarrow\left[\theta_{1}, \theta_{2}\right]$, and $h:\left[a_{\gamma}, 0\right] \rightarrow\left[\theta_{3}, \frac{1}{b}\right]$. Then, let $u_{1}\left(a, \alpha_{a}\right), u_{2}\left(a, \alpha_{a}\right)$ denote the first, second recommendations of types $x(a), g(a)$ (for all $a \in[-2,0]$ ), and let $v_{1}\left(a, \alpha_{0}\right), v_{2}\left(a, \alpha_{0}\right)$ denote the first, second recommendations of types $(z(a), h(a))$ (for all $a \in\left[a_{\gamma}, 0\right]$ ). With this notation, Groups I,II, III described in the text are as follows:

$$
\begin{aligned}
\text { Group } I & =\left\{z(a), h(a) \mid a \in\left[a_{\gamma}, 0\right]\right\} \\
\text { Group II } & =\left\{x(a), g(a) \mid a \in[-2,0], \text { and } \exists a^{\prime} \in\left[a_{\gamma}, 0\right] \text { with } v_{1}\left(a^{\prime}, \alpha_{0}\right)=u_{1}\left(a, \alpha_{a}\right)\right\} \\
\text { Group III } & =\{x(a), g(a) \mid a \in[-2,0], \text { and } x(a), g(a) \notin \text { Group II }\}
\end{aligned}
$$

In our proposed equilibrium construction, each Group $I$ pair $\{z(a), h(a)\}$ recommends $v_{1}\left(a, \alpha_{0}\right)$ for $2 \alpha_{0}$ periods, then $v_{2}\left(a, \alpha_{0}\right)$ for $T-\tau-2 \alpha_{0}$ periods, then reveals the truth at time $T-\tau$; each Group $I I$ pair $\{x(a), g(a)\}$ recommends $u_{1}\left(a, \alpha_{a}\right)$ for $2 \alpha_{a}$ periods, then $u_{2}\left(a, \alpha_{a}\right)$ for $2\left(1-\alpha_{a}\right)$ periods, then separates and reveals the truth for the final $T-2$ periods; and moreover, the recommendation $u_{1}\left(a, \alpha_{a}\right)$ coincides with the recommendation $v_{1}\left(a^{\prime}, \alpha_{0}\right)$ of some Group $I$ pair $\left\{z\left(a^{\prime}\right), h\left(a^{\prime}\right)\right\}$. Group $I I I$ is identical to Group $I I$, except that their recommendations do not coincide with those of any Group I pair.

We also specify the following off-path strategy for the expert: if the DM ever deviates, by rejecting a recommendation that the expert made, then (i) if the expert himself has not previously

\footnotetext{
${ }^{12}$ It is trivial to check exactly as we did in previous examples that these indifference conditions suffice for global incentive compatibility.
} 
deviated: send no further recommendations (equivalently, repeat the current recommendation in all subsequent periods). And (ii) if the expert has observably deviated in the past, behave as if the deviation did not occur. (For example, if he sends the initial recommendation $u_{1}\left(0, \alpha_{0}\right)$ prescribed for types $\{x(0), g(0)\}$, but then follows this with anything other than recommendation $u_{2}\left(0, \alpha_{0}\right)$ at time $2 \alpha_{0}$, subsequently behave as if the deviation never occurred and he indeed sent $u_{2}\left(0, \alpha_{0}\right)$ at time $\left.2 \alpha_{0}\right)$.

\section{D.1 Optimality for the Expert}

We prove that the expert wishes to follow the prescribed recommendation strategy via three propositions. Proposition D1 specifies strategies and beliefs for the DM such that the expert has no incentive to send an out-of-equilibrium recommendation sequence, so we can restrict to making sure that he does not wish to mimic any other type. Proposition D2 shows that in the prescribed revelation phase, the expert indeed finds it optimal to reveal the truth, provided that there have been no previous deviations. It remains only to show that the expert has no incentive to deviate prior to the prescribed revelation phase - by mimicking the initial recommendations of some other type - which we show in Proposition D3.

We specify the following strategy and beliefs for the DM:

DM Strategy and Beliefs: If there are no detectable deviations by the expert (i.e., he sends the equilibrium recommendation sequence for some type $\left.\theta \in\left[0, \frac{1}{b}\right]\right)$, then follow all recommendations, using Bayes' rule to assign beliefs at each information set. Following deviations: (i) If the expert observably deviates at time 0 (sending an off-path initial recommendation), subsequently adopt the strategy/beliefs that would follow if the expert had instead sent the recommendation $u_{1}\left(0, \alpha_{0}\right)$ prescribed for types $\{x(0), g(0)\}$; (ii) If the expert observably deviates on his 2 nd recommendation (i.e., if an initial recommendation $u_{1}\left(a, \alpha_{a}\right)$ (or $v_{1}\left(a, \alpha_{0}\right)$ ) is followed by something other than $u_{2}\left(a, \alpha_{a}\right)$ (or $\left.v_{2}\left(a, \alpha_{0}\right)\right)$, ignore it as an error, and subsequently adopt the strategy/beliefs that would follow had the deviation not occurred; (iii) If the expert deviates observably in the revelation phase, ignore it as an error, assigning probability 1 to the lowest type in the current information set, and accordingly choosing this as the myopically optimal action; (iv) And finally, if the DM himself deviates, rejecting some recommendation by the expert, then he subsequently maintains the current (at time of deviation) beliefs, anticipating that the expert will subsequently repeat the current (at time of deviation) recommendation, and ignoring any other recommendations as errors.

\section{D.1.1 Expert Optimality: Off-Path Behavior}

Proposition D1: Under the above strategy and beliefs prescribed for the DM, the expert has no incentive to choose an off-path recommendation sequence.

Proof of Proposition D1: Follows trivially from the specified strategy and beliefs for the DM: (i) a deviation at time zero is equivalent to mimicking type $x(0)$ (who recommends $u_{1}\left(0, \alpha_{0}\right)$ at time $t=0$ ); (ii) a deviation on the 2nd recommendation has no effect, since the DM ignores it; (iii) a deviation in the revelation phase, if there have been no previous deviations, is equivalent to mimicking the strategy of the lowest type in the DM's current (pre-revelation) information set; and (iv) if the DM has previously deviated, then (by point (iv) of the above strategy-belief specification) he will chose whichever action was myopically optimal at the time of deviation, regardless of the expert's message; therefore, babbling is optimal for the expert, since his message has no effect on the DM's action.

\section{D.1.2 Expert Optimality: Truth Revelation Phase}

Proposition D2: In the prescribed revelation phase, (i) if there have been no previous deviations by the DM, then the expert finds it optimal to reveal the truth; (ii) if the DM has ever deviated, then the expert finds it optimal to babble (eg. by remaining silent).

Proof of Proposition D2: Part (ii) follows immediately from Proposition D1 (iv). For part (i): our specification of the expert strategy is such that at time $2 \alpha_{0}$, the DM's information set contains at most two types: either a pair $\{x(a), g(a)\}$ (in which case he plans to choose $g(a)$ if the expert 
recommends $g(a), x(a)$ otherwise), or a pair $\{z(a), h(a)\}$ (in which case he plans to choose $h(a)$ if the expert recommends it, $z(a)$ otherwise). So, it suffices to show that each type would rather tell the truth than mimic his partner, which requires (in our rescaled state space) simply that all paired types be at least 2 units apart. By (10) we have

$$
\begin{aligned}
& \min _{a \in[-2,0]}|g(a)-x(a)|=\theta_{2}-\theta_{1} \\
& \min _{a \in\left[a_{\gamma}, 0\right]}|h(a)-z(a)|=\theta_{3}-\theta_{2}
\end{aligned}
$$

And by $(9), \theta_{2}-\theta_{1}=\left(a_{\gamma}-2+2 e^{-a_{\gamma}}\right) \geq 8\left(\right.$ for $\left.b<\frac{1}{61}\right)$, and $\theta_{3}-\theta_{2}=2$.

\section{D.1.3 Expert Optimality: Initial Recommendations}

Propositions D1,D2 imply that once the expert has sent the initial recommendation $\left(u_{1}\right.$ or $\left.v_{1}\right)$ prescribed for some type $\theta$, it is optimal to follow also the continuation recommendations prescribed for that type. So, the only time when it could possibly be profitable to deviate is at time $t=0$ : we need to make sure that each type $\theta$ prefers to send the proposed equilibrium sequence of recommendations, rather than the sequence prescribed for any other type $\theta^{\prime} .{ }^{13}$ We now choose parametrizations of functions $x, g, z, h$, along with action function $u_{1}, u_{2}, v_{1}, v_{2}$, which guarantee that the expert indeed finds it optimal to send the prescribed initial recommendation:

Proposition D3: Let the action functions and type parametrizations be as follows:

$$
\begin{aligned}
& x(a)=\theta_{3}+a-\theta_{3} e^{a}, g(a)=\theta_{3}+a, z(a)=\frac{1}{b}+a-2 e^{a-a_{\gamma}}, h(a)=\frac{1}{b}+a \\
& u_{1}\left(a, \alpha_{a}\right)=\theta_{3}+K-\frac{T-2}{2} a-\sqrt{\frac{1-\alpha_{a}}{\alpha_{a}}} \sqrt{T-2} \sqrt{C_{u}+a\left(K-\frac{T}{4} a\right)} \\
& u_{2}\left(a, \alpha_{a}\right)=\theta_{3}+K-\frac{T-2}{2} a+\sqrt{\frac{\alpha_{a}}{1-\alpha_{a}}} \sqrt{T-2} \sqrt{C_{u}+a\left(K-\frac{T}{4} a\right)} \\
& v_{1}\left(a, \alpha_{0}\right)=\theta_{3}+\frac{2 K-\tau\left(a-a_{\gamma}\right)}{T-\tau}-\frac{\sqrt{\frac{\tau\left(T-\tau-2 \alpha_{0}\right)}{\alpha_{0}}} \sqrt{\frac{(T-\tau)(T-2)}{\tau} C_{u}+\left(\frac{T-\tau-2}{\tau}\right) K^{2}+2 K\left(a-a_{\gamma}\right)-\frac{T}{2}\left(a-a_{\gamma}\right)^{2}}}{T-\tau} \\
& v_{2}\left(a, \alpha_{0}\right)=\theta_{3}+\frac{2 K-\tau\left(a-a_{\gamma}\right)}{T-\tau}+\frac{\sqrt{\frac{4 \tau \alpha_{0}}{T-\tau-2 \alpha_{0}}} \sqrt{\frac{(T-\tau)(T-2)}{\tau} C_{u}+\left(\frac{T-\tau-2}{\tau}\right) K^{2}+2 K\left(a-a_{\gamma}\right)-\frac{T}{2}\left(a-a_{\gamma}\right)^{2}}}{T-\tau}
\end{aligned}
$$

for constants $C_{u}, K$, and for now taking $T, \alpha_{0}, \alpha_{a}$ as given ( $T$ is the horizon, and $\alpha_{a}, \alpha_{0}$ relate to the duration of recommendations $u_{1}, v_{1}$ as described in the strategies above). Also set $\tau$ (length of the revelation phase for types in Group I) according to

$$
\frac{\tau}{T-2}=\beta \equiv \frac{\left(\theta_{2}-\theta_{1}\right)\left(\theta_{2}-\theta_{1}-2\right)}{\left(\theta_{4}-\theta_{1}\right)\left(\theta_{4}-\theta_{1}-2\right)}
$$

Then, for all types $\theta, \theta^{\prime} \in\left[0, \frac{1}{b}\right]$, expert type $\theta$ prefers his equilibrium recommendation sequence to that sent by type $\theta^{\prime}$, and in particular has no incentive to deviate at time $t=0$ from the prescribed strategy.

\section{Proof of Proposition D3:}

Let $D_{u}\left(\theta^{\prime} \mid \theta\right)$ denote the disutility to type $\theta$ from following the recommendation sequence prescribed for a type $\theta^{\prime} \in\left[0, \theta_{1}\right] \cup\left[\theta_{2}, \theta_{3}\right]$, and let $D_{v}\left(\theta^{\prime} \mid \theta\right)$ denote the disutility to type $\theta$ from following

\footnotetext{
${ }^{13}$ This is what the text refers to as "providing incentives to join the right separable group". We need to make sure, for example, that type $\theta=0$ prefers to induce the action sequence $\left(u_{1}(0), u_{2}(0), 0\right)$, rather than e.g. the sequence that type $\theta^{\prime} \neq 0$ is supposed to send; by Propositions D1,D2, the choice to follow a different recommendation sequence can only be made at time $t=0$.
} 
the strategy prescribed for a type $\theta^{\prime} \in\left[\theta_{1}, \theta_{2}\right] \cup\left[\theta_{3}, \frac{1}{b}\right]$. The proof proceeds through two main Lemmas. Lemma D3.1 proves that the expert strategy is "locally" incentive compatible: for each interval $\left[\theta_{i}, \theta_{i+1}\right], i \in\{0,1,2,3\}$, no expert type $\theta \in\left[\theta_{i}, \theta_{i+1}\right]$ wishes to mimic any other type $\theta^{\prime} \in\left[\theta_{i}, \theta_{i+1}\right]$ from the same interval. Lemma D3.2 proves that the expert strategy is also "globally" incentive compatible: no expert type wishes to mimic any type $\theta^{\prime}$ from any other interval. The proofs will use calculations obtained below in Lemmas D3.3 and D3.4.

Lemma D3.1 (Local IC): For each interval $\left[\theta_{i}, \theta_{i+1}\right]$, with $i=0,1,2,3$, and any pair of types $\theta, \theta^{\prime} \in\left[\theta_{i}, \theta_{i+1}\right]$, the disutility to type $\theta$ from mimicking type $\theta^{\prime}$ is (weakly) increasing in $\left|\theta^{\prime}-\theta\right|$, thus minimized when $\left|\theta^{\prime}-\theta\right|=0$. Therefore, for each $\theta \in\left[\theta_{i}, \theta_{i+1}\right]$, truth-telling is (weakly) better than mimicking any other type in the interval.

\section{Proof of Lemma D3.1:}

Differentiating disutility expressions (20), (21), (22), and (23) (obtained below in Lemma D3.3) gives

$$
\begin{aligned}
\frac{d D_{u}(x(a) \mid \theta)}{d x(a)} & =\frac{d D_{u}(g(a) \mid \theta)}{d x(a)}+\frac{2(T-2)(x(a)-\theta-1) x^{\prime}(a)-2(T-2)(g(a)-\theta-1) g^{\prime}(a)}{x^{\prime}(a)} \\
& =0+2(T-2)\left(\theta_{3}+a-\theta_{3} e^{a}-\theta-1-\frac{\theta_{3}+a-\theta-1}{1-\theta_{3} e^{a}}\right)(\text { by } \\
& =2(T-2)\left(\frac{\theta_{3} e^{a}}{\theta_{3} e^{a}-1}\right)(x(a)-\theta) \\
\frac{d D_{v}(z(a) \mid \theta)}{d z(a)} & =\frac{d D_{v}(h(a) \mid \theta) / d a}{z^{\prime}(a)}+\frac{2 \tau(z(a)-\theta-1) z^{\prime}(a)-2 \tau(h(a)-\theta-1) h^{\prime}(a)}{z^{\prime}(a)} \\
\frac{d D_{u}(g(a) \mid \theta)}{d g(a)} & =0 \\
\frac{d D_{v}(h(a) \mid \theta)}{d h(a)} & =0
\end{aligned}
$$

Consider first a type $\theta \in\left[0, \theta_{1}\right]$. By (16), noting that $\frac{\theta_{3} e^{a}}{\theta_{3} e^{a}-1}>0$ (since $\theta_{3} e^{a} \geq \theta_{3} e^{-2}=\theta_{2}-\theta_{1} \geq$ 8 , by Proposition D2), we see that $\frac{d D_{u}(x(a) \mid \theta)}{d x(a)}$ has the same sign as $(x(a)-\theta)$. So if $x(a)-\theta>0$, then $D_{u}(x(a) \mid \theta)$ is increasing in $x(a)$, thus increasing in $(x(a)-\theta)$; while if $x(a)-\theta<0$, then $D_{u}(x(a) \mid \theta)$ is increasing in $(-x(a))$, thus increasing in $\theta-x(a)$. Combined, these establish that $D_{u}(x(a) \mid \theta)$ is strictly increasing in $|x(a)-\theta|$, as desired.

Next consider a type $\theta \in\left[\theta_{1}, \theta_{2}\right]$. By (17), noting that $\left(\frac{2 e^{a-a_{\gamma}}}{2 e^{a-a_{\gamma}}-1}\right)>0$ (since $a \in\left[a_{\gamma}, 0\right]$ implies $2 e^{a-a_{\gamma}} \geq 2$ ), we see that $\frac{d D_{v}(z(a) \mid \theta)}{d z(a)}$ has the same sign as $z(a)-\theta$, and is thus positive (disutility increasing in $z(a)-\theta$ ) if $z(a)>\theta$, and negative (disutility increasing in $\theta-z(a)$ ) if $z(a)<\theta$. Combined, these establish that $D_{u}(z(a) \mid \theta)$ is strictly increasing in $|z(a)-\theta|$, as desired.

By (18) and (19), the disutility to type $\theta$ from mimicking a type $g(a) \in\left[\theta_{2}, \theta_{3}\right]$ or $h(a) \in\left[\theta_{3}, \frac{1}{b}\right]$ is independent of the particular type $g(a), h(a)$ chosen. Thus, $D_{u}(g(a) \mid \theta)$ is weakly increasing (in fact constant) in $|g(a)-\theta|$, and $D_{u}(h(a) \mid \theta)$ is weakly increasing (constant) in $|h(a)-\theta|$, completing the proof.

Lemma D3.2: For every interval $\left[\theta_{i}, \theta_{i+1}\right](i=0,1,2,3)$, and every $\theta \in\left[\theta_{i}, \theta_{i+1}\right]$, following the prescribed (truthful) recommendation sequence is better than mimicking any type $\theta^{\prime}$ drawn from any other interval $\left[\theta_{j}, \theta_{j+1}\right]$ with $j \neq i$.

Proof of Lemma D3.2:

Consider first a type $\theta \in\left[0, \theta_{1}\right]$. By Lemma D3.1, truth-telling is better than mimicking any other type $\theta^{\prime} \in\left[0, \theta_{1}\right]$, in particular type $\theta_{1}=x(-2)$. By Lemma D3.4 (i) (below), type $\theta \in\left[0, \theta_{1}\right]$ prefers type $x(-2)$ 's sequence to type $z(0)$ 's sequence; and by Lemma D3.1, it is better to mimic 
type $z(0)=\theta_{1}$, than any other type $z(a) \in\left(\theta_{1}, \theta_{2}\right]$ (since $z(a)>\theta$ implies that $D_{v}(z(a) \mid \theta)$ is increasing in $z(a)-\theta)$; together, these establish that mimicking a type $\theta^{\prime} \in\left[\theta_{1}, \theta_{2}\right]$ is not optimal. By Lemma D3.4 (ii), type $\theta \leq \theta_{2}$ prefers $z\left(a_{\gamma}\right)$ 's sequence (right endpoint of $\left[\theta_{1}, \theta_{2}\right]$ ) to $g(-2)$ 's sequence (left endpoint of $\left[\theta_{2}, \theta_{3}\right]$ ); and by Lemma D3.1, disutility to type $\theta$ from mimicking type $g(a) \in\left[\theta_{2}, \theta_{3}\right]$ is independent of $a$; together, this implies that type $\theta$ also does not want to mimic any type $g(a) \in\left[\theta_{2}, \theta_{3}\right]$. And finally, by Lemma D3.4 (iii), type $\theta \leq \theta_{2}$ prefers the sequence prescribed for type $g(0)$ (right endpoint of $\left[\theta_{2}, \theta_{3}\right]$ ) to that prescribed for type $h\left(a_{\gamma}\right)$ (left endpoint of $\left[\theta_{3}, \frac{1}{b}\right]$ ), which (by Lemma D3.1) yields the same utility as mimicking any other type $h(a) \in\left[\theta_{3}, \frac{1}{b}\right]$, thus it is not optimal to mimic any type $h(a) \in\left[\theta_{3}, \frac{1}{b}\right]$. This establishes that type $\theta \in\left[0, \theta_{1}\right]$ does not wish to mimic any type $\theta^{\prime}$ from any other interval.

Next consider type $\theta \in\left[\theta_{1}, \theta_{2}\right]$. By Lemma D3.1, truth-telling is better than mimicking any other type $z(a) \in\left[\theta_{1}, \theta_{2}\right]$, in particular type $z(0)=\theta_{1}$; by Lemma D3.4 (i), type $\theta \geq \theta_{1}$ prefers the sequence prescribed for type $z(0)$, to that prescribed for type $x(-2)$; and by Lemma D3.1, it is better to mimic $x(-2)$ (right endpoint of $\left.\left[0, \theta_{1}\right]\right)$ than any other type $x(a) \in\left[0, \theta_{1}\right]$, since $D_{u}(x(a) \mid \theta)$ is increasing in $|\theta-x(a)|$ and we have here $\theta>x(a)$; together, this implies that type $\theta$ does not wish to mimic any type $\theta^{\prime} \in\left[0, \theta_{1}\right]$. The proof that he doesn't wish to mimic any type $g(a) \in\left[\theta_{2}, \theta_{3}\right]$ or $h(a) \in\left[\theta_{3}, \frac{1}{b}\right]$ is identical to the one given in the previous paragraph.

Now consider a type $\theta \in\left[\theta_{2}, \theta_{3}\right]$. As explained in the previous two paragraphs, following the truthful recommendation sequence yields the same utility as mimicking any other type $g(a) \in$ $\left[\theta_{2}, \theta_{3}\right]$ or $h(a) \in\left[\theta_{3}, \theta_{4}\right]$, so we just need to make sure that it is not optimal to mimic types $\theta^{\prime} \in\left[0, \theta_{1}\right] \cup\left[\theta_{1}, \theta_{2}\right]$. By Lemma D3.4 (ii), type $\theta \geq \theta_{2}$ prefers type $g(-2)$ 's sequence (left endpoint of $\left[\theta_{2}, \theta_{3}\right]$ ) to type $z\left(a_{\gamma}\right)$ 's sequence (right endpoint of $\left[\theta_{1}, \theta_{2}\right]$ ); by Lemma D3.1, such a type $\theta \geq \theta_{2}$ also prefers type $z\left(a_{\gamma}\right)$ 's sequence to the one prescribed for any other (further-away) type $z(a) \in\left[\theta_{1}, \theta_{2}\right]$; combined, this establishes that mimicking a type $z(a) \in\left[\theta_{1}, \theta_{2}\right]$ is not optimal. By Lemma D3.4 (i), it is better to mimic type $z(0)$ 's sequence than $x(-2)$ 's sequence, which in turn is better (by Lemma D3.1) than any other type $x(a)$ 's sequence. Thus, it is not optimal to mimic any type $x(a) \in\left[0, \theta_{1}\right]$, completing the proof for types $\theta \in\left[\theta_{2}, \theta_{3}\right]$.

The argument that types $\theta \in\left[\theta_{3}, \frac{1}{b}\right]$ don't wish to mimic types from other intervals is identical to the proof in the previous paragraph (for types $\theta \in\left[\theta_{2}, \theta_{3}\right]$ ).

This completes the proof of Lemma D3.2.

\section{D.1.4 Expert Optimality: Preliminary Calculations}

Lemma D3.3: Given the type parametrizations and action functions given in Proposition D3, disutility expressions $D_{u}\left(\theta^{\prime} \mid \theta\right), D_{v}\left(\theta^{\prime} \mid \theta\right)$ are given by

$$
\begin{aligned}
D_{u}(x(a) \mid \theta) & =D_{u}(g(a) \mid \theta)+(T-2)(x(a)-\theta-1)^{2}-(T-2)(g(a)-\theta-1)^{2} \\
D_{u}(g(a) \mid \theta) & =T\left(\theta_{3}-\theta-1\right)^{2}+4 K\left(\theta_{3}-\theta-1\right)+2 K^{2}+2(T-2) C_{u} \\
D_{v}(z(a) \mid \theta) & =D_{v}(h(a) \mid \theta)-2 \tau(h(a)-z(a))\left(\frac{h(a)+z(a)}{2}-\theta-1\right) \\
D_{v}(h(a) \mid \theta) & =2 K^{2}+2(T-2) C_{u}+4\left(\theta_{3}-\theta-1\right) K+T\left(\theta-\theta_{3}+1\right)^{2}
\end{aligned}
$$

\section{Proof of Lemma D3.3:}

The disutility $D_{u}(g(a) \mid \theta)$ to expert type $\theta$ from following the strategy prescribed for type $g(a) \in$ 
$\left[\theta_{2}, \theta_{3}\right]$, using $(11),(12)$, is

$$
\begin{aligned}
& 2 \alpha_{a}\left(u_{1}(a)-\theta-1\right)^{2}+2\left(1-\alpha_{a}\right)\left(u_{2}(a)-\theta-1\right)^{2}+(T-2)(g(a)-\theta-1)^{2} \\
= & 2\left(1-\alpha_{a}\right)\left(\theta_{3}+K-\frac{T-2}{2} a-\theta-1+\sqrt{\frac{\alpha_{a}}{1-\alpha_{a}}} \sqrt{T-2} \sqrt{C_{u}+a\left(K-\frac{T}{4} a\right)}\right) \\
& +2 \alpha_{a}\left(\theta_{3}+K-\frac{T-2}{2} a-\theta-1-\sqrt{\frac{1-\alpha_{a}}{\alpha_{a}}} \sqrt{T-2} \sqrt{C_{u}+a\left(K-\frac{T}{4} a\right)}\right)^{2}+(T-2)(x(a)-\theta-1)^{2}
\end{aligned}
$$

Expanding gives 14

$D_{u}(g(a) \mid \theta)=2\left(\theta_{3}-\theta-1+K-\frac{T-2}{2} a\right)^{2}+2(T-2)\left(C_{u}+a\left(K-\frac{T}{4} a\right)\right)+(T-2)\left(\theta_{3}+a-\theta-1\right)^{2}$

If we now expand this expression, the coefficients on $a^{2}, a$ reduce to zero (this is due to our choice $\left.g(a)=\theta_{3}+a\right)$, leaving

$$
D_{u}(g(a) \mid \theta)=2\left(\theta_{3}-\theta-1+K\right)^{2}+2(T-2) C_{u}+(T-2)\left(\theta_{3}-\theta-1\right)^{2}
$$

which rearranges to expression $(21)$.

The disutility to type $\theta$ from following the strategy prescribed for type $x(a) \in\left[0, \theta_{1}\right], D_{u}(x(a) \mid \theta)$, is given by (24), just replacing $g(a)$ with $x(a)$ : this gives the desired expression $(20)$.

The disutility to type $\theta$ from following the strategy prescribed for type $h(a) \in\left[\theta_{3}, \theta_{4}\right]$ is

$$
D_{v}(h(a) \mid \theta)=2 \alpha_{0}\left(v_{1}(a)-\theta-1\right)^{2}+\left(T-\tau-2 \alpha_{0}\right)\left(v_{2}(a)-\theta-1\right)^{2}+\tau(h(a)-\theta-1)^{2}
$$

Again, the coefficients on the square root terms in $v_{1}, v_{2}$ were chosen to make both disutility and average action independent of $\alpha_{0}$ : substituting (13),(14) into the above expression and expanding, we get

$$
\begin{aligned}
D_{v}(h(a) \mid \theta)= & (T-\tau)\left(\theta_{3}+\frac{2 K+\tau a_{\gamma}}{T-\tau}-\frac{\tau}{T-\tau} a-\theta-1\right)^{2}+\tau(h(a)-\theta-1)^{2} \\
& +2 \tau\left(\frac{(T-2)}{\tau} C_{u}+\frac{\left(\frac{T-\tau-2}{\tau}\right) K^{2}+2 K\left(a-a_{\gamma}\right)-\frac{T}{2}\left(a-a_{\gamma}\right)^{2}}{T-\tau}\right)
\end{aligned}
$$

Substituting in $h(a)=\theta_{4}+a$, using $\theta_{3}=\theta_{4}+a_{\gamma}$, and expanding, we find (this is due to our choice $\left.h^{\prime}(a)=1\right)$ that the coefficients on both $a^{2}$, a reduce to zero, so that our expression simplifies further to (23). Finally, using the fact that the strategies for types $h(a), z(a)$ differ only in the revelation phase, so

$$
D_{v}(h(a) \mid \theta)-D_{v}(z(a) \mid \theta)=\tau(h(a)-\theta-1)^{2}-\tau(z(a)-\theta-1)^{2}
$$

we obtain (22). This completes the proof.

Lemma D3.4: (utility at the endpoints)

Under the expressions given in Proposition D3, we have that (i) endpoint $\theta_{1}=x(-2)=z(0)$ : type $\theta$ (weakly) prefers type $x(-2)$ 's recommendation sequence to $z(0)$ 's sequence iff $\theta \in\left[0, \theta_{1}\right]$; (ii) endpoint $\theta_{2}=z\left(a_{\gamma}\right)=g(-2)$ : type $\theta$ prefers $z\left(a_{\gamma}\right)$ 's sequence to $g(-2)$ 's sequence iff $\theta \in\left[0, \theta_{2}\right]$; and (iii) endpoint $\theta_{3}=g(0)=h\left(a_{\gamma}\right)$ : all types are indifferent between the sequences sent by types

\footnotetext{
${ }^{14}$ Note that the coefficients on the square roots were chosen to make this independent of $\alpha_{a}$, as mentioned in Appendix E - Derivations.
} 
$g(0), h\left(a_{\gamma}\right){ }^{15}$

Proof of Lemma D3.4:

At $\theta_{1}=x(-2)=z(0)$, we have (using the expressions in Lemma D3.3 and simplifying) that $D_{v}(z(0) \mid \theta)-D_{u}(x(-2) \mid \theta)$ equals

$$
(T-2)\left(\theta_{2}-\theta_{1}\right)\left(\theta_{1}+\theta_{2}-2 \theta-2\right)-\tau\left(\theta_{4}-\theta_{1}\right)\left(\theta_{4}+\theta_{1}-2 \theta-2\right)
$$

Using $\tau\left(\theta_{4}-\theta_{1}\right)=(T-2) \frac{\left(\theta_{2}-\theta_{1}\right)\left(\theta_{2}-\theta_{1}-2\right)}{\left(\theta_{4}-\theta_{1}-2\right)}$ (by (15)), this simplifies to

$$
D_{v}(z(0) \mid \theta)-D_{u}(x(-2) \mid \theta)=2(T-2)\left(\theta_{2}-\theta_{1}\right)\left(\theta_{4}-\theta_{2}\right)\left(\frac{\theta_{1}-\theta}{\theta_{4}-\theta_{1}-2}\right)
$$

This is negative, meaning that type $\theta$ prefers $z(0)$ 's strategy to $x(-2)$ 's strategy, iff $\theta>\theta_{1}$, thus establishing part (i).

At $\theta_{2}=g(-2)=z\left(a_{\gamma}\right)$, we have (by $(22)$ and $(21)$

$$
\begin{aligned}
D_{u}(g(-2) \mid \theta)-D_{v}\left(z\left(a_{\gamma}\right) \mid \theta\right) & =\tau\left(\theta_{3}-\theta_{2}\right)\left(\theta_{3}+\theta_{2}-2 \theta-2\right) \\
& \left.=4 \tau\left(\theta_{2}-\theta\right) \text { (using } \theta_{3}-\theta_{2}=2\right)
\end{aligned}
$$

This is negative, meaning that type $\theta$ prefers $g(-2)$ 's strategy to $z\left(a_{\gamma}\right)$ 's strategy, iff $\theta>\theta_{2}$, proving part (ii).

At $\theta_{3}$, we have (by (21) and (23)),

$$
D_{u}(g(0) \mid \theta)-D_{v}\left(h\left(a_{\gamma}\right) \mid \theta\right)=0
$$

so that all types are indifferent between the strategies prescribed for type $g(0)=\theta_{3}, h\left(a_{\gamma}\right)=\theta_{3}$, as desired to complete the proof.

\section{D.2 Optimality for the DM}

Let the expert strategy be as specified in the previous subsection, using the action functions and parametrizations from Proposition D3, with $\tau=\beta(T-2)$ as in (15). Recall that we had the following free parameters: constants $K, C_{u}$, the horizon $T$, a number $\alpha_{0} \in[0,1]$, and numbers $\alpha_{a} \in[0,1] \forall a \in[-2,0]$. We wish to show that the specified strategies constitute a fully revealing PBE: since we established expert optimality in the previous section, and since the beliefs and offpath strategies specified for the DM (see Proposition D1) trivially satisfy all PBE requirements, all that remains is to prove that the DM's on-path strategy is optimal.

Recall the timeline presented in Figure 1 (section 5). It is immediately clear that during the revelation phase, when the expert's recommendation is equal (with probability 1 ) to the true state, the DM indeed finds it optimal to follow the recommendation. In between time $2 \alpha_{0}$ (when Group $I$ separates from Group $I I$ by switching to $v_{2}$ ) and the revelation phase, no new information is revealed, but any failure by the decision-maker to follow the expert's recommendations will result in the expert subsequently babbling, rather than revealing the truth. So, the best possible deviation is to choose the myopically optimal action in all subsequent periods, and the strongest incentive to do so occurs at the earliest time that new information is revealed (when the "reward phase", revelation of the truth, is furthest away). So to prove DM optimality, we need only show that he does not want to deviate to the myopically optimal action either at time $t=0$, or at time $t=2 \alpha_{0}$ if he learns that he is in fact facing a Group $I$ pair. We summarize this as:

\footnotetext{
${ }^{15}$ For example, consider part (i). In our construction, type $\theta_{1}$ is both the right endpoint $x(-2)$ of the interval $\left[0, \theta_{1}\right]$, and the left endpoint $z(0)$ of the interval $\left[\theta_{1}, \theta_{2}\right]:$ part (i) says that type $\theta_{1}$ is indifferent between the two sequences prescribed for his type, and that everyone below $\theta_{1}$ prefers the strategy of type $x(-2)$, everyone above $\theta_{1}$ prefers the strategy of type $z(0)$.
} 
Observation D4: If the DM cannot gain by deviating at time $t \in\left\{0,2 \alpha_{0}\right\}$, then the prescribed strategy is optimal.

\section{D.2.1 Optimality for the DM: Outline and Parameter Choices}

Given $T, \alpha_{0}$ (and with $\tau=\beta(T-2)$ as specified by (15), we set the constants $C_{u}, K$ according to

$$
\begin{aligned}
C_{u} & =\frac{1-\alpha_{0}}{\alpha_{0}} \frac{K^{2}}{T-2} \\
K & =\frac{\alpha_{0} \tau a_{\gamma}\left(1+\sqrt{\frac{\left(T-2 \alpha_{0}\right)(T-\tau)}{2 \tau \alpha_{0}}}\right)}{\left(T-\tau-2 \alpha_{0}\right)}
\end{aligned}
$$

And choose a horizon $T$ satisfying

$$
\begin{cases}\text { if } \beta a_{\gamma}^{2}<8: & T-\tau=4 \Leftrightarrow T=T_{1} \\ \text { if } \beta a_{\gamma}^{2}>8: & 6 \leq T \leq \min \left\{T_{1}, T_{2}\right\}\end{cases}
$$

where $T_{1}=\frac{2(2-\beta)}{1-\beta}$, and $T_{2}=\frac{2 \widehat{\phi}^{2}}{1-\widehat{\phi}^{2}(1-\beta)}$, with $\widehat{\phi}$ solving $\ln \left(\frac{\widehat{\phi}^{2}}{1+\widehat{\phi}}\right)=1 \Leftrightarrow \widehat{\phi} \cong 3.4959$. Note that with $\beta$ as specified in (15), $\beta a_{\gamma}^{2}<8 \Leftrightarrow a_{\gamma} \gtrsim-3.18$. In the range $\beta a_{\gamma}^{2}>8$, we also have $\min \left\{T_{1}, T_{2}\right\}=T_{2}$ whenever $\beta>.95825$, and that $T_{1}>11.5, T_{2}>24$. All proofs use $\alpha_{0}$ near 1 when $\beta a_{\gamma}^{2}<8$, and $\alpha_{0}$ near 0 when $\beta a_{\gamma}^{2}>8$. The parameter $\alpha_{a}$ (relating to the time $2 \alpha_{a}$ at which Group $I I$ and $I I I$ pairs $\{x(a), g(a)\}$ switch from $u_{1}$ to $\left.u_{2}\right)$ may depend on the specific pair $\{x(a), g(a)\}$, but is chosen in Lemma D7.1 to satisfy $\alpha_{0} \leq \alpha_{a} \leq 1 \forall a$.

The constants $C_{u}, K$ from (28), (29) are chosen in Lemma D7.1, in order to facilitate the overlap of the functions $u_{1}, v_{1}$. The need for this overlap is as follows: the DM's gain to following the expert's advice is large at information sets containing only a Group II or III pair, but would be negative at time $t=0$, for all priors, if his information set contained only a Group I pair. ${ }^{16}$ So, for the equilibrium to work, we need to make sure that each Group I pair's initial message coincides with that of a Group II pair, and then ensure (via the prior and construction details) that the weight the DM places on the Group $I I$ pair is high enough to make him want to follow the recommendation. Specifically, Lemma D7.1 proves that under (28), (29), if we set $T \leq T_{1}$, with equality if $\beta a_{\gamma}^{2}<8$, then for every $a^{\prime} \in\left[a_{\gamma}, 0\right]$, there exists $a \in[-2,0]$ and $\alpha_{a} \in\left[\alpha_{0}, 1\right]$ such that each Group $I$ recommendation $v_{1}\left(a^{\prime}, \alpha_{0}\right)$ coincides with Group $I I$ recommendation $u_{1}\left(a, \alpha_{a}\right)$ (and moreover both recommendations are real-valued); the result imposes a lower bound $\underline{\alpha_{0}}<1$ on $\alpha_{0}$ when $\beta a_{\gamma}^{2}<8$ (so in particular it holds for $\alpha_{0}$ near 1), and an upper bound $\overline{\alpha_{0}}>\overline{0}$ on $\alpha_{0}$ when $\beta a_{\gamma}^{2}>8$ (so in particular it holds for $\alpha_{0}$ near zero).

Proposition D6 shows that for a range of priors, the DM's gain to deviating at time $t=0$ (or later) is strictly negative at any information set containing only a group $I I$ or $I I I$ pair $\{x(a), g(a)\}$. This then implies also that at time $t=0$, if he gets a message $v_{1}\left(a, \alpha_{0}\right)$ which could have been sent by either a Group $I I$ pair (in which case he wants to follow the advice) or a Group $I$ pair (in which case he might want to reject the advice), he will find it optimal to follow the recommendation as long as his posterior beliefs assign a high enough weight to the Group II pair, so we conclude that there exist beliefs for which the DM has no incentive to deviate at time $t=0$. It is also Proposition D6 that places an upper bound on the biases $b$ for which the equilibrium works. The proof specifically uses posteriors near 50-50, but in fact the range of priors that work is quite large.

\footnotetext{
${ }^{16}$ It may in fact be shown (see Section E.2) that this is necessarily true of any fully revealing construction: the expert's local + global IC constraints imply a sufficiently large distortion in some interval of types' (and their partners') recommendations that, if the DM were certain that he was facing one of these pairs, he would rather forego learning the exact truth than follow their advice.
} 
Proposition D5 shows that if the expert sends a message $v_{2}\left(a, \alpha_{0}\right)$ at time $t=2 \alpha_{0}$, thus revealing to the DM that he is facing a Group $I$ pair $\{z(a), h(a)\}$, then there exist posteriors on the two types for which the DM will find it optimal to choose the action $v_{2}\left(a, \alpha_{0}\right)$. Specifically, we prove that if $\beta a_{\gamma}^{2}<8$ the incentive constraints are slack in the limit as $\alpha_{0} \rightarrow 1$, and if $\beta a_{\gamma}^{2}>8$ the incentive constraints are slack in the limit as $\alpha_{0} \rightarrow 0$. It is also this result which places the additional upper bound $T \leq T_{2}$ (from (30)) on the horizon.

Finally, Proposition D7 completes the proof, by proving that there exist priors which generate the posteriors used Propositions D5, D6.

Finally, we briefly comment on the timeline. First, note that Theorem 1 places only a lower bound on the horizon $T^{*}$, whereas the constraint (30) in fact also places an upper bound on the horizon (as $b \rightarrow 0 \Leftrightarrow a_{\gamma} \rightarrow-\infty$, the constraint $T \leq T_{2}$ from Proposition D5 binds, with $\left.T_{2} \rightarrow 24.443\right)$. However, the construction may trivially be extended for larger horizons in two ways: (i) add a babbling phase at the beginning; or (ii) scale everything up. All that matters is the ratios - the length of each initial recommendation compared to the length of the revelation phase - so e.g. the analysis for a $T$-period equilibrium (where Groups $I I, I I I$ reveal the truth at time 2, Group $I$ at time $T-\tau$ ) is identical to the anlysis for a $T \lambda$-period equilibrium (with Groups $I I, I I I$ revealing the truth at time $2 \lambda$, Group $I$ at time $(T-\tau) \lambda$, for $\lambda$ any positive number). ${ }^{17}$ It should also be noted that the times at which the expert instructs the DM to change his action - namely, times $2 \alpha_{0}, 2 \alpha_{a}, T-\tau-$ are not necessarily integers in our construction. In a continuous time setting, where action changes can be made frequently, this clearly poses no problem. In a discrete time setting, it is possible to scale everything up to obtain integers. ${ }^{18}$

\section{D.2.2 Optimality for the DM: Deviations at time $t=2 \alpha_{0}$}

Proposition D5: Suppose that the DM receives recommendation $v_{2}(a)$ at time $t=2 \alpha_{0}$, and assigns probabilities $q_{a}, 1-q_{a}$ to the two types $z(a), h(a)$ in his information set. Under (28), (29), and (30), there exists a range of values for $\frac{q_{a}}{1-q_{a}}$ such that (i) if $\beta a_{\gamma}^{2}<8$, the DM's gain to deviating at time $2 \alpha_{0}$ is strictly negative for $\alpha_{0}$ near 1 , and (ii) if $\beta a_{\gamma}^{2}>8$, the gain to deviating is strictly negative for $\alpha_{0}$ near zero.

\section{Proof of Proposition D5:}

If the DM follows recommendation $v_{2}(a)$ (expecting to choose this action until time $T-\tau$, then learn the truth), his expected disutility is

$$
\left(T-\tau-2 \alpha_{0}\right)\left(q_{a}\left(v_{2}(a)-z(a)\right)^{2}+\left(1-q_{a}\right)\left(v_{2}(a)-h(a)\right)^{2}\right)+\tau(0)
$$

The best possible deviation is to instead choose myopically optimal action $q_{a} z(a)+\left(1-q_{a}\right) h(a)$ in all remaining $T-2 \alpha_{0}$ periods, for disutility

$$
\begin{aligned}
& \left(T-2 \alpha_{a}\right)\left(q_{a}\left(q_{a} z(a)+\left(1-q_{a}\right) h(a)-z(a)\right)^{2}+\left(1-q_{a}\right)\left(q_{a} z(a)+\left(1-q_{a}\right) h(a)-h(a)\right)^{2}\right) \\
= & (T-2 \alpha) q_{a}\left(1-q_{a}\right)(h(a)-z(a))^{2}
\end{aligned}
$$

\footnotetext{
${ }^{17}$ This follows immediately from the derivations in Appendix E.
}

${ }^{18}$ This is not completely trivial, due to the fact that the coefficient $\frac{\tau}{T-2}$ specified in (15) may not be rational, but this can be easily modified via minor changes to the construction. Specifically, given bias $b$, the easiest way to make $\beta$ rational is to change the $h$-function (specifying how Group $I$ types are paired), as follows: first, choose a rational

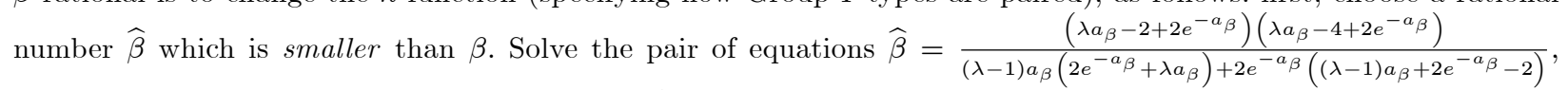
$\frac{1}{b}=\left(\lambda a_{\beta}-2+2 e^{-a_{\beta}}\right) e^{2}-\lambda a_{\beta}$ for $\lambda$ and $a_{\beta}(\widehat{\beta}<\beta$ will give $\lambda>1$, as required by the expert's S.O.C.'s $)$. In all analysis, replace $a_{\gamma}$ with $a_{\beta}$, and change the $h$-function from $h(a)=\frac{1}{b}+a$ to $h(a)=\frac{1}{b}+\lambda a$; this requires changing the endpoints specified in (9) to $\theta_{3}=\frac{1}{b}+\lambda a_{\beta}$, with (as before) $\theta_{2}=\theta_{3}-2, \theta_{1}=\theta_{2}-\theta_{3} e^{-2}$. To satisfy the expert's modified local IC constraints, simply plug the new $h$-function into the recommendation functions $v_{1}, v_{2}$ specified by (54). To satisfy the expert's modified global IC constraints, replace $\beta$ with $\widehat{\beta}$, and change the constant $C_{v}$ (from $(57))$ in the $v$-functions (specified in (54)) by replacing $a_{\gamma}$ with $a_{\beta}$, and also subtracting the term $\frac{(\lambda-1)}{2} a_{\beta}\left(a_{\beta}+2\right)$; no change to the constant $k_{v}$. For $\widehat{\beta}$ sufficiently close to $\beta$, the changes to the analysis for the DM are negligible. 
So to prove the Proposition, we need to show that there are $q_{a}$ 's s.t. the following inequality holds:

$$
\begin{aligned}
0 & \geq\left(q_{a}\left(v_{2}(a)-z(a)\right)^{2}+\left(1-q_{a}\right)\left(v_{2}(a)-h(a)\right)^{2}\right)-\frac{\left(T-2 \alpha_{0}\right)}{\left(T-\tau-2 \alpha_{0}\right)} q_{a}\left(1-q_{a}\right)(h(a)-z(a))^{2} \\
& =q_{a}\left(2\left(\frac{v_{2}(a)-h(a)}{h(a)-z(a)}\right)+1\right)+\left(\frac{v_{2}(a)-h(a)}{h(a)-z(a)}\right)^{2}-\frac{T-2 \alpha_{0}}{T-\tau-2 \alpha_{0}} q_{a}\left(1-q_{a}\right)
\end{aligned}
$$

This is easiest to satisfy (RHS is minimized) at

$$
q_{a}=q_{a}^{*} \equiv \frac{1}{2}-\frac{\left(\frac{v_{2}(a)-h(a)}{h(a)-z(a)}\right)+\frac{1}{2}}{\frac{T-2 \alpha_{0}}{T-\tau-2 \alpha_{0}}}
$$

where (31) becomes

$$
\begin{aligned}
\frac{\phi^{2}-1}{4 \phi^{2}}\left(2\left(\frac{v_{2}(a)-h(a)}{h(a)-z(a)}\right)+\phi+1\right)\left(2\left(\frac{v_{2}(a)-h(a)}{h(a)-z(a)}\right)+1-\phi\right) & \leq 0 \\
\text { with } \phi^{2} & =\frac{T-2 \alpha_{0}}{T-\tau-2 \alpha_{0}}
\end{aligned}
$$

Thus, a sufficient condition for the DM's IC constraint (31) to hold is

$$
-\phi-1 \leq 2\left(\frac{v_{2}(a)-h(a)}{h(a)-z(a)}\right) \leq \phi-1
$$

which implies both that (33) holds (so the DM does not want to deviate), and that $q_{a}^{*}$ (from (32)) is indeed a probability, since we have

$$
\begin{aligned}
& 2\left(\frac{v_{2}(a)-h(a)}{h(a)-z(a)}\right)+1+\phi^{2}>2\left(\frac{v_{2}(a)-h(a)}{h(a)-z(a)}\right)+1+\phi \geq 0 \Rightarrow q_{a}^{*}<1 \\
& 2\left(\frac{v_{2}(a)-h(a)}{h(a)-z(a)}\right)+1-\phi^{2}<2\left(\frac{v_{2}(a)-h(a)}{h(a)-z(a)}\right)+1-\phi \leq 0 \Rightarrow q_{a}^{*}=0
\end{aligned}
$$

Substituting $C_{u}=\frac{1-\alpha_{0}}{\alpha_{0}} \frac{K^{2}}{T-2}\left(\right.$ from (28)) into (14), we obtain that $v_{2}\left(a, \alpha_{0}\right)-h(a)$ equals

$$
\theta_{3}+\frac{2 K-\tau\left(a-a_{\gamma}\right)}{T-\tau}+\frac{\sqrt{\frac{4 \tau \alpha_{0}}{T-\tau-2 \alpha_{0}}} \sqrt{\frac{(T-\tau)(T-2)}{\tau} \frac{1-\alpha_{0}}{\alpha_{0}} \frac{K^{2}}{T-2}+\left(\frac{T-\tau-2}{\tau}\right) K^{2}+2 K\left(a-a_{\gamma}\right)-\frac{T}{2}\left(a-a_{\gamma}\right)^{2}}}{T-\tau}-h(a)
$$

Simplifying, and using $h(a)-z(a)=2^{a-a_{\gamma}}$ and $\theta_{3}-\theta_{4}=a_{\gamma}($ from (10) and (9)), this implies that

$$
\begin{aligned}
2 \frac{v_{2}\left(a, \alpha_{0}\right)-h(a)}{h(a)-z(a)} & =\frac{2 K-T\left(a-a_{\gamma}\right)+\sqrt{\frac{2 \alpha_{0}}{T-\tau-2 \alpha_{0}}} \sqrt{\frac{2 \frac{T-\tau-2 \alpha_{0}}{\alpha_{0}} K^{2}+2 \tau\left(2 K\left(a-a_{\gamma}\right)-\frac{T}{2}\left(a-a_{\gamma}\right)^{2}\right)}{(T-\tau) e^{a-a_{\gamma}}}}}{}=\frac{k-t y+\sqrt{k^{2}+\left(\phi^{2}-t\right)\left(2 k y-t y^{2}\right)}}{e^{y}}
\end{aligned}
$$

where $k \equiv \frac{2 K}{T-\tau}, t \equiv \frac{T}{T-\tau}$, and $y \equiv a-a_{\gamma}$ (with $\phi$ defined in (34)). So by (35), we wish to show that 
for all $y \in\left[0,-a_{\gamma}\right]$,

$$
\frac{k-t y+\sqrt{k^{2}+\left(\phi^{2}-t\right)\left(2 k y-t y^{2}\right)}}{e^{y}} \in[-\phi-1, \phi-1]
$$

By construction, the value of $K$ specified in (29) sets the square root portion of $v_{1}, v_{2}$ equal to zero at $a=0 \Leftrightarrow y=-a_{\gamma}$ (see Lemma D7.1), so we have

$$
k=a_{\gamma}\left(\phi^{2}-t+\phi \sqrt{\phi^{2}-t}\right)
$$

We first prove (ii). Suppose $\beta a_{\gamma}^{2}>8$, in which case Lemma D7.1 requires $\frac{T-\tau}{2} \leq 2$, and $\alpha_{0} \leq \overline{\alpha_{0}}$ (for some $\bar{\alpha}_{0} \in(0,1)$ ). Consider what happens as $\alpha_{0} \rightarrow 0$ (in which case we trivially satisfy $\left.\alpha_{0} \leq \overline{\alpha_{0}}\right)$ : then $\phi^{2}-t=\frac{T-2 \alpha_{0}}{T-\tau-2 \alpha_{0}}-\frac{T}{T-\tau} \rightarrow 0$, implying also that $k \rightarrow 0$, so we have

$$
\begin{aligned}
& \min _{y \in\left[0,-a_{\gamma}\right]}\left(k-t y+\sqrt{k^{2}+\left(\phi^{2}-t\right)\left(2 k y-t y^{2}\right)}+(1+\phi) e^{y}\right) \rightarrow \min _{y \in\left[0,-a_{\gamma}\right]}\left(-\phi^{2} y+(\phi+1) e^{y}\right) \\
& \max _{y \in\left[0,-a_{\gamma}\right]}\left(k-t y+\sqrt{k^{2}+\left(\phi^{2}-t\right)\left(2 k y-t y^{2}\right)}+(1-\phi) e^{y}\right) \rightarrow \max _{a \in\left[0,-a_{\gamma}\right]}\left(-\phi^{2} y+(1-\phi) e^{y}\right)
\end{aligned}
$$

In the second line, the limit is clearly decreasing in $y$ (noting that $\phi \rightarrow \sqrt{\frac{T}{T-\tau}}>1$ ), thus maximized at $y=0$ : here, the value is $1-\phi$, the desired upper bound in (36). In the first line, note that if we set $\phi$ s.t.

$$
(\phi+1) e>\phi^{2} \Leftrightarrow 1>\ln \frac{\phi^{2}}{\phi+1} \Leftrightarrow \phi<3.4959
$$

then the limiting expression $\left(-\phi^{2} y+(1+\phi) e^{y}\right)$, which is minimized at $y=\ln \frac{\phi^{2}}{\phi+1}<1$, has a minimum value of

$$
-\phi^{2} \ln \frac{\phi^{2}}{\phi+1}+\phi^{2}=\phi^{2}\left(1-\ln \frac{\phi^{2}}{\phi+1}\right)
$$

which is positive, thus trivially satisfying the desired lower bound in (36).

So to complete the proof of (ii), we just need to show that for $\alpha_{0}$ near zero, we can find a horizon $T$ which satisfies both the requirement $\Delta \leq 2$ (from Proposition D6 and Lemma D7.1), as well as

$$
\sqrt{\frac{T}{T-\tau}}=\lim _{\alpha \rightarrow 0} \phi<3.4959
$$

Using $\tau=\beta(T-2)$, which implies $T=\frac{2(\Delta-\beta)}{1-\beta}$, the above inequality binds if $\beta>\frac{(3.4959)^{2}-1}{(3.4959)^{2}-\frac{1}{2}}=$ 0.95734 , and otherwise is implied by $\Delta \leq 2 \Leftrightarrow T \leq \frac{4-2 \beta}{1-\beta}$; this gives the horizon constraint (30) specified in the Proposition.

Finally we prove (i). Suppose $\beta a_{\gamma}^{2}<8$, in which case Lemma D7.1 uses $\frac{T-\tau}{2} \equiv \Delta=2$ and requires $\alpha_{0} \geq \underline{\alpha_{0}}$, for some $\underline{\alpha_{0}} \in[0,1]$. So, set $\Delta=2$, and consider what happens as $\alpha_{0} \rightarrow 1$ : then

$$
t \rightarrow \frac{T}{4}=\frac{2-\beta}{2(1-\beta)}, \phi^{2} \rightarrow \frac{T-2}{T-\tau-2}=\frac{1}{1-\beta}, k \rightarrow \frac{\beta a_{\gamma}}{2(1-\beta)}\left(1+\sqrt{\frac{2}{\beta}}\right)
$$


Define $f(y) \equiv k-t y+\sqrt{k^{2}+\left(\phi^{2}-t\right)\left(2 k y-t y^{2}\right)}$; we want to prove that

$$
-(1+\phi) \leq \frac{f(y)}{e^{y}} \leq \phi-1 \text { for all } y \in\left[0,-a_{\gamma}\right]
$$

To this end, note that

$$
\begin{aligned}
f^{\prime}(y) & =-t+\frac{\left(\phi^{2}-t\right)(k-t y)}{\sqrt{k^{2}+\left(\phi^{2}-t\right)\left(2 k y-t y^{2}\right)}}<0\left(\text { by } \phi^{2}-t>0, y>0, \text { and } k<0\right) \\
f^{\prime \prime}(y) & =\frac{-\left(\phi^{2}-t\right) k^{2} \phi^{2}}{\left(k^{2}+\left(\phi^{2}-t\right)\left(2 k y-t y^{2}\right)\right)^{\frac{3}{2}}}<0
\end{aligned}
$$

This implies that $f(y)$ reaches a maximum (over $y \in\left[0,-a_{\gamma}\right]$ ) at $y=0$, and lies above the straight line connecting the points $(0, f(0))$ and $\left(-a_{\gamma}, f\left(-a_{\gamma}\right)\right)$ : since we have $f\left(-a_{\gamma}\right)=k+t a_{\gamma}$ (the square root term is zero here by construction), and $f(0)=k+\sqrt{k^{2}}=0$, this line $\widetilde{f}$ is given by

$$
\widetilde{f}(y)-\widetilde{f}(0)=\frac{\widetilde{f}\left(-a_{\gamma}\right)-\widetilde{f}(0)}{-a_{\gamma}}(y-0) \Rightarrow \widetilde{f}(y)=\left(\frac{-k}{a_{\gamma}}-t\right) y
$$

Substituting in $k=\frac{\beta a_{\gamma}}{2(1-\beta)}\left(1+\sqrt{\frac{2}{\beta}}\right)$, this becomes $\widetilde{f}(y)=-\left(\frac{1+\sqrt{\frac{\beta}{2}}}{1-\beta}\right) y$. Then, the upper bound in (37) follows from the fact that

$$
\max _{y \in\left[0,-a_{\gamma}\right]} \frac{f(y)}{e^{y}} \leq \frac{\max _{y \in\left[0,-a_{\gamma}\right]} f(0)}{\min _{y \in\left[0,-a_{\gamma}\right]} e^{y}}=0 \leq \phi-1
$$

while the lower bound in (37) follows from

$$
\min _{y \in\left[0,-a_{\gamma}\right]} \frac{f(y)}{e^{y}} \geq \min _{y \in\left[0,-a_{\gamma}\right]} \frac{\tilde{f}(y)}{e^{y}}=\min _{y \in\left[0,-a_{\gamma}\right]}-\left(\frac{1+\sqrt{\frac{\beta}{2}}}{1-\beta}\right) \frac{y}{e^{y}}=\frac{-\left(1+\sqrt{\frac{\beta}{2}}\right)}{(1-\beta) e}
$$

So, using $\phi=\frac{1}{\sqrt{1-\beta}}$, to show that $\min _{y \in\left[0,-a_{\gamma}\right]} \frac{f(y)}{e^{y}}+(1+\phi) \geq 0$, it suffices to show that

$$
\frac{-\left(1+\sqrt{\frac{\beta}{2}}\right)}{(1-\beta) e}+1+\sqrt{\frac{1}{1-\beta}} \geq 0 \Leftrightarrow e \geq \frac{\left(1+\sqrt{\frac{\beta}{2}}\right)}{1-\beta+\sqrt{1-\beta}}
$$

Noting that the RHS is clearly increasing in $\beta$, it may be easily verified numerically that the above expression holds for all $\beta \leq 0.82035$, which is implied by the range under consideration $\left(\beta a_{\gamma}^{2}<8 \Leftrightarrow a_{\gamma} \gtrsim-3.18\right.$ implies $\left.\bar{\beta}<0.8\right)$. This completes the proof for $a_{\gamma} \geq-3.2 \Leftrightarrow \beta a_{\gamma}^{2}<8$, showing that if we set $T=\frac{4-2 \beta}{1-\beta}$, then all DM IC constraints are slack in the limit as $\alpha_{0} \rightarrow 1$ (and so, by continuity, hold for all $\alpha_{0}$ sufficiently high, which satisfy the bounds in Lemma D7.1). This completes the proof.

\section{D.2.3 Optimality for the DM: Deviations at time $t=0$}


Proposition D6: For all $b<\frac{1}{61} \Leftrightarrow a_{\gamma} \leq-1.7726$, and for all $a \in[-2,0]$, there exists a range of values $p_{a}$ such that if the DM assigns probabilities $p_{a}, 1-p_{a}$ to types $x(a), g(a)$, then his gain to deviating at information set $\{x(a), g(a)\}$ is strictly negative at any time $t \geq 0$.

Proof of Proposition D6: As explained in Observation D4, it suffices to prove that the gain to deviating is negative at time $t=0$. Substituting $x(a)=\theta_{3}+a-\theta_{3} e^{a}$ and $g(a)=\theta_{3}+a$ into (58), we obtain that the DM's gain to deviating at information set $\{x(a), g(a)\}$, if he assigns probability $p_{a}$ to type $x(a)$, is

$$
\begin{aligned}
& 2 p_{a}\left(K-\frac{T}{2} a+\theta_{3} e^{a}\right)^{2}+2\left(1-p_{a}\right)\left(K-\frac{T}{2} a\right)^{2}+2(T-2) a\left(K-\frac{T}{4} a\right)-T p_{a}\left(1-p_{a}\right)\left(\theta_{3} e^{a}\right)^{2}+2(T-2) C \\
= & 2 K^{2}+4 K\left(p_{a} \theta_{3} e^{a}-a\right)+T\left(p_{a} \theta_{3} e^{a}-a\right)^{2}-(T-2) p_{a}\left(\theta_{3} e^{a}\right)^{2}+2(T-2) C_{u}
\end{aligned}
$$

If we make $p_{a}=p$ constant, the derivative of this expression w.r.t. $a$ is

$$
\begin{aligned}
& 2\left(p \theta_{3} e^{a}-1\right)\left[2 K-T a+T p \theta_{3} e^{a}-\frac{(T-2) p\left(\theta_{3} e^{a}\right)^{2}}{\left(p \theta_{3} e^{a}-1\right)}\right] \\
\leq & 2\left(p \theta_{3} e^{a}-1\right)\left[2 K-T a-((1-p) T-2) \theta_{3} e^{a}\right]
\end{aligned}
$$

So, it suffices to choose a value $p$ s.t. (39) is negative $\forall a$ (implying that the DM's gain to deviating is largest-making the IC constraint most difficult to satisfy-at $a=-2$ ), and such that this maximal gain to deviating at $a=-2$ is negative. In the proof below, we will set $p \leq \frac{1}{2}$, in which case (30) implies that $(1-p) T-2>0$, from which it immediately follows that the square bracketed term in (39) is decreasing in $a$, thus negative everywhere if it is negative at $a=-2$. Using this and rearranging, (39) requires

$$
\theta_{3} e^{-2} \geq \frac{2 K+2 T}{(1-p) T-2}
$$

For the maximal gain to deviating to be negative, evaluate (38) at $a=-2$ and solve for $\theta_{3} e^{-2}$, to get

$$
\theta_{3} e^{-2} \geq \frac{(2 K+2 T)+\sqrt{\frac{2(T-2)}{p}\left((1-p) K^{2}+4 K+2 T+((1-p) T-2) C_{u}\right)}}{(1-p) T-2}
$$

This trivially implies the inequality (40). So, it is sufficient to choose a value $p \leq \frac{1}{2}$ which satisfies (41), and then set $p_{a}=p \forall a \in[-2,0]$.

If $a_{\gamma}>-1.7786 \Leftrightarrow \theta_{3} e^{-2}<8$, this is impossible (see Appendix E: this is what yields the upper bound $b \cong \frac{1}{61}$ on the biases for which our construction works).

If $a_{\gamma} \in[-2,-1.7786]$, set $p=\frac{1}{4}$; by (30) and (29), recalling that in this range we use $\alpha_{0} \rightarrow 1$, we have

$$
T=\frac{4-2 \beta}{1-\beta}, K \rightarrow \frac{\beta a_{\gamma}}{1-\beta}\left(1+\sqrt{\frac{2}{\beta}}\right), C_{u} \rightarrow 0
$$

Substituting these values into (41), along with $\theta_{3} e^{-2}=a_{\gamma}-2+2 e^{-a_{\gamma}}$, and simplifying, (41) becomes

$$
0 \geq a_{\gamma}-2+2 e^{-a_{\gamma}}-8\left(\frac{1+\sqrt{\frac{2}{\beta}}}{1+\frac{2}{\beta}}\right)\left(\left(\frac{a_{\gamma}}{2}-1+\sqrt{\frac{2}{\beta}}\right)+\sqrt{\frac{3}{4}\left(\frac{a_{\gamma}^{2}}{1-\beta}\right)+\frac{4}{\beta} \frac{\left(a_{\gamma}-1+\sqrt{\frac{2}{\beta}}\right)}{\left(1+\sqrt{\frac{2}{\beta}}\right)}}\right)
$$

Using $\beta=\frac{\left(a_{\gamma}-2+2 e^{-a_{\gamma}}\right)\left(a_{\gamma}-4+2 e^{-a_{\gamma}}\right)}{2 e^{-a_{\gamma}}\left(2 e^{-a_{\gamma}}-2\right)}$ from (15), this holds for $a_{\gamma} \in[-2,-1.7726]$, as shown in the graph below. 
If $a_{\gamma} \leq-2$, set $p=\frac{1}{2}$, so that (41) becomes

$$
\theta_{3} e^{-2} \geq 4 \frac{(K+T)+\sqrt{\left(\frac{T-2}{2}\right)\left((K+4)^{2}+4(T-4)+(T-4) C_{u}\right)}}{T-4}
$$

Substituting in (28) and (29), this becomes

$$
\frac{\theta_{3} e^{-2}}{4} \geq \frac{\frac{\alpha_{0} \tau a_{\gamma}\left(1+\sqrt{\frac{\left(T-2 \alpha_{0}\right)(T-\tau)}{2 \tau \alpha_{0}}}\right)}{\left(T-\tau-2 \alpha_{0}\right)}+T+\sqrt{\frac{\left(T-4+2 \alpha_{0}\right)}{2 \alpha_{0}}\left(\frac{\alpha_{0} \tau a_{\gamma}\left(1+\sqrt{\frac{\left(T-2 \alpha_{0}\right)(T-\tau)}{2 \tau \alpha_{0}}}\right)}{\left(T-\tau-2 \alpha_{0}\right)}\right)^{2}+(T-2)\left(\frac{4 \alpha_{0} \tau a_{\gamma}\left(1+\sqrt{\frac{\left(T-2 \alpha_{0}\right)(T-\tau)}{2 \tau \alpha_{0}}}\right)}{\left(T-\tau-2 \alpha_{0}\right)}+2 T\right)}}{T-4}
$$

If $\beta a_{\gamma}^{2}>8 \Leftrightarrow a_{\gamma} \lesssim-3.18$, our proof uses $\alpha_{0} \rightarrow 0$ : in this case, taking limits in (43) yields

$$
\frac{\theta_{3} e^{-2}}{4} \geq \frac{T+\sqrt{T(T-2)\left(\frac{(T-4)}{4} \frac{\beta a_{\gamma}^{2}}{(T-\tau)}+2\right)}}{T-4}
$$

Since $\beta<1$ and $T-\tau-2=(1-\beta)(T-2)>0$ (by (15)), an upper bound on the RHS is attained at $\beta=1, T-\tau=2$; with this, using $\theta_{3} e^{-2}=a_{\gamma}-2+2 e^{-a_{\gamma}}$, and rearranging, it is sufficient to prove that

$$
\left(\frac{T-4}{4}\left(a_{\gamma}-2+2 e^{-a_{\gamma}}\right)-T\right)^{2} \geq T(T-2)\left(\frac{(T-4)}{8} a_{\gamma}^{2}+2\right)
$$

Expanding and dividing through by $T(T-4)$, this becomes

$$
\left(\frac{T-4}{4 T}\right)\left(\frac{a_{\gamma}-2+2 e^{-a_{\gamma}}}{2}\right)^{2}-\left(\frac{a_{\gamma}-2+2 e^{-a_{\gamma}}}{2}\right)-\frac{(T-2)}{8} a_{\gamma}^{2}-1 \geq 0
$$

The derivative of the LHS is increasing in $T$, so that the expression becomes easier to satisfy as $T$ increases, whenever

$$
\left(\frac{a_{\gamma}-2+2 e^{-a_{\gamma}}}{2 T}\right)^{2}-\frac{a_{\gamma}^{2}}{8} \geq 0 \Leftrightarrow T \leq \frac{a_{\gamma}-2+2 e^{-a_{\gamma}}}{\frac{-a_{\gamma}}{\sqrt{2}}}
$$

Using (15), this is implied by the restriction $T \leq \frac{4-2 \beta}{1-\beta}$ from (30) (whenever $a_{\gamma} \lesssim-1.3691$ ), and so our expression is most difficult to satisfy when $T$ is small; choosing $T \geq 6$ is sufficient for the range $a_{\gamma} \lesssim-3.18$ under consideration, yielding the lower bound on $T$ given in (30) .

Finally, for $a_{\gamma} \in[-3.18,-2]$, we proved Proposition D5 using $\alpha_{0} \rightarrow 1$ (which implies $C_{u} \rightarrow 0$, $\left.K \rightarrow \frac{\beta a_{\gamma}}{1-\beta}\left(1+\sqrt{\frac{2}{\beta}}\right)\right)$ and $T=\frac{4-2 \beta}{1-\beta}$. Substituting into (43), we need

$$
\left.\left(\frac{2}{\beta}\right)\left(\beta a_{\gamma}\left(1+\sqrt{\frac{2}{\beta}}\right)+4-2 \beta+\sqrt{\left((1-\beta)\left(\frac{\beta a_{\gamma}}{1-\beta}\left(1+\sqrt{\frac{2}{\beta}}\right)+4\right)^{2}+8 \beta\right.}\right)\right)-a_{\gamma}+2-2 e^{-a_{\gamma}} \leq 0
$$

Substituting in $\beta=\frac{\left(a_{\gamma}-2+2 e^{-a_{\gamma}}\right)\left(a_{\gamma}-4+2 e^{-a_{\gamma}}\right)}{2 e^{-a_{\gamma}}\left(2 e^{-a_{\gamma}}-2\right)}$ (from (15)), this holds as desired for $a_{\gamma} \in[-3.18,-2]$, as shown in the Fig D below. 


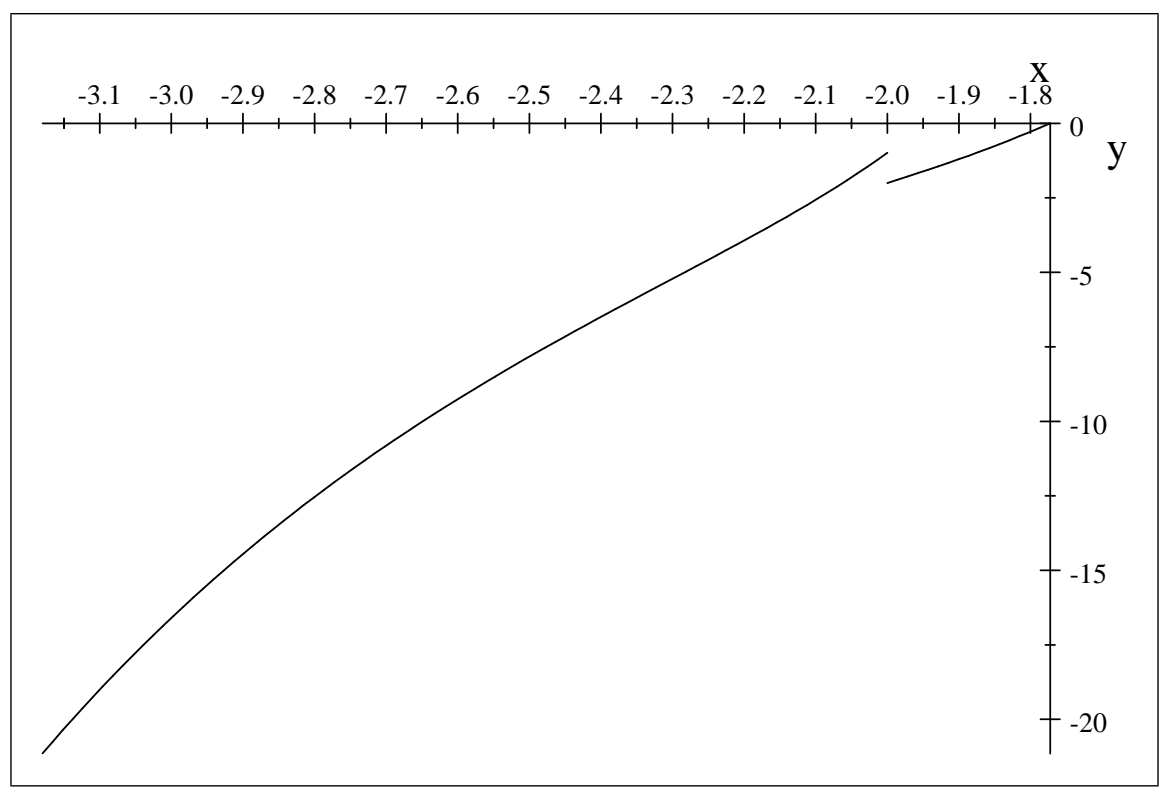

Fig D: DM's ex ante gain to deviating

(This shows (44) for $a_{\gamma} \in[-3.18,-2]$, and (42) for $a_{\gamma} \in[-1.7726]$, both strictly negative as desired). This completes the proof of Proposition D6. ${ }^{19}$

\section{D.2.4 Optimality for the DM: Completing the Proof}

As explained at the beginning of this section, it remains only to prove that there are many priors $\mu$ generating posteriors which satisfy the conditions in Propositions D5, D6, which we prove here: Proposition D7: For any continuous functions $p:[-2,0] \rightarrow[0,1], q:\left[a_{\gamma}, 0\right] \rightarrow[0,1]$, and $r:$ $\left[a_{\gamma}, 0\right] \rightarrow[0,1]$, there exists a density $f$ over the state space such that, in our construction, a Bayesian DM will hold the following beliefs: (i) $\operatorname{Pr}(x(a) \mid\{x(a), g(a)\})=p(a)$; (ii) $\operatorname{Pr}(z(a) \mid\{z(a), h(a)\})=$ $q(a)$; (iii) $\operatorname{Pr}(\{z(a), h(a)\} \mid\{z(a), h(a), x(\widehat{a}), g(\widehat{a})\}=r(a)$.

Proof of Proposition D7: Bayesian beliefs satisfy

$$
\begin{aligned}
\frac{\operatorname{Pr}(x(a) \mid\{x(a), g(a)\})}{\operatorname{Pr}(g(a) \mid\{x(a), g(a)\})} & =\frac{f(x(a))}{f(g(a))}\left(\theta_{3} e^{a}-1\right) \\
\frac{\operatorname{Pr}(z(a) \mid\{z(a), h(a)\})}{\operatorname{Pr}(h(a) \mid\{z(a), h(a)\})} & =\frac{f(z(a))}{f(h(a))}\left(2 e^{a-a_{\gamma}}-1\right) \\
\frac{\operatorname{Pr}(\{z(a), h(a)\})}{\operatorname{Pr}(\{x(\widehat{a}), g(\widehat{a})\})} & =\frac{f(z(a))}{f(\widehat{x}(a))} \frac{p(\widehat{a})}{q(a)} \frac{2 e^{a-a_{\gamma}}-1}{\theta_{3} e^{\widehat{a}(a)}-1} \cdot \frac{1}{\left|\widehat{a}^{\prime}(a)\right|}
\end{aligned}
$$

where $\widehat{a}(a)=u_{1}^{-1}\left(v_{1}\left(a^{\prime}\right)\right)$ (as explained in Section E.2.1). We want the first expression to equal $\frac{p(a)}{1-p(a)}$, the second expression to equal $\frac{q(a)}{1-q(a)}$, and the third expression to equal $\frac{r(a)}{1-r(a)}$. It is straightforward to construct such a density $f$ : for example, for each $a \in[-2,0]$, set

$$
f(x(a))=\frac{1}{M}
$$

with $M$ a constant to be determined (this assigns probabilities to $\left[0, \theta_{1}\right]$ ). Then, assign probabilities

\footnotetext{
${ }^{19}$ It should be noted that, for brevity of exposition, we made $p$ equal a constant $p_{a} \in\left\{\frac{1}{4}, \frac{1}{2}\right\}$, which works throughout the range. But for each specific value of $a_{\gamma}$, the set of beliefs that work is in fact much wider than a small interval around $p_{a}$.
} 
to types $g(a) \in\left[\theta_{2}, \theta_{3}\right]$ using this and the first expression above, i.e. set

$$
f(g(a))=f(x(a))\left(\theta_{3} e^{a}-1\right)\left(\frac{1-p(a)}{p(a)}\right)=\frac{\left(\theta_{3} e^{a}-1\right)}{M} \frac{1-p(a)}{p(a)}
$$

Next, for each $a \in\left[a_{\gamma}, 0\right]$, set $f(z(a))$ to satisfy the 3 rd bound above, i.e.

$$
f(z(a))=\frac{\frac{q(a)}{p(\widehat{a})} \frac{\theta_{3} e^{\widehat{a}(a)}-1}{2 e^{a-a \gamma}-1} \cdot\left|\widehat{a}^{\prime}(a)\right|}{M} \frac{r(a)}{1-r(a)}
$$

(This assigns a prior for types $z(a) \in\left[\theta_{1}, \theta_{2}\right]$ ). And similarly, now use this and the second expression above to assign beliefs to types $h(a) \in\left[\theta_{3}, \theta_{4}\right]$. Finally, choose $M$ so that the total measure of the type space integrates to 1 (This is possible since $(11),(13)$ imply that $u_{1}, v_{1}$ and their derivatives w.r.t. $a$ are finite and non-zero except perhaps at a single point $a$, from which it follows that $\left|\widehat{a}^{\prime}(a)\right|$ is bounded (again, except perhaps at a single point $a$ ), which implies that all of the desired probabilities $\mu(x(a)), \mu(g(a)), \mu(z(a))$ specified above are bounded - in particular finite numbers divided by a number $M$ - so that integrating over the state space will yield a finite number divided by $M$. Choose $M$ so that this number equals 1.)

\section{D.2.5 Optimality for the DM: Preliminary Calculations}

Lemma D7.1: Define

$$
\left[\underline{\alpha_{0}}, \overline{\alpha_{0}}\right] \equiv\left[\frac{(T-\tau)+\beta a_{\gamma} \sqrt{\frac{T-\tau}{2 \beta}}}{2+\left(-\beta a_{\gamma}\right)\left(\frac{2(T-2)}{T}+\frac{T-4}{T} \sqrt{\frac{T-\tau}{2 \beta}}\right)}, 1-(T-\tau-2)\left(\sqrt{\frac{1}{2 \beta(T-\tau)}}-\frac{1}{-\beta a_{\gamma}}\right)\right]
$$

Then (i) $T-\tau \leq 4$ and $\beta a_{\gamma}^{2}>8$ implies that $\underline{\alpha}_{0}<0<\overline{\alpha_{0}}$, (ii) $T-\tau=4$ and $\beta a_{\gamma}^{2}<8$ implies $\underline{\alpha}_{0}<1<\bar{\alpha}_{0}$, (iii) $\alpha_{0} \in\left[\alpha_{0}, \overline{\alpha_{0}}\right]$ implies that $u_{t}, v_{t}$ are both real-valued, and (iv) $\alpha_{0} \in\left[\alpha_{0}, \overline{\alpha_{0}}\right]$ implies that for every $a \in\left[a_{\gamma}, 0\right]$, there exists a pair $\left(\widehat{a}(a), \alpha_{a}\right)$, with $\alpha_{a} \geq \alpha_{0}$ and $\widehat{a}(a) \in[-2,0]$, such that

$$
u_{1}\left(\widehat{a}(a), \alpha_{a}\right)=v_{1}\left(a, \alpha_{0}\right)
$$

Proof of Lemma D7.1: We first prove (i). If $T-\tau \leq 4<\frac{\beta a_{\gamma}^{2}}{2}$, then

$$
(T-\tau)<\sqrt{\frac{\beta a_{\gamma}^{2}(T-\tau)}{2}}=-\beta a_{\gamma} \sqrt{\frac{T-\tau}{2 \beta}}
$$

Thus the numerator in the expression for $\underline{\alpha}_{0}$ is negative, while the denominator is clearly positive, so we obtain $\underline{\alpha}_{0}<0$. To complete the proof of (i), we need to show that $\bar{\alpha}_{0}>0$ : we have

$$
\begin{aligned}
T-\tau \in[1,4] \Rightarrow(T-\tau-2)^{2} & \leq(T-\tau) \\
& \left.<2 \beta(T-\tau) \text { for } \beta>\frac{1}{2} \text { (implied by } \beta a_{\gamma}^{2}>8\right) \\
\Rightarrow T-\tau-2 & <\frac{1}{\sqrt{\frac{1}{2 \beta(T-\tau)}}}<\frac{1}{\left(\sqrt{\frac{1}{2 \beta(T-\tau)}}-\frac{1}{-\beta a_{\gamma}}\right)}
\end{aligned}
$$

and so $\bar{\alpha}_{0}=1-(T-\tau-2)\left(\sqrt{\frac{1}{2 \beta(T-\tau)}}-\frac{1}{-\beta a_{\gamma}}\right)>0$, as desired. 
For part (ii), set $T-\tau=4:$ then

$$
\beta a_{\gamma}^{2}<8 \Rightarrow \frac{\beta a_{\gamma}^{2}}{2}<T-\tau \Rightarrow \sqrt{\frac{1}{2 \beta(T-\tau)}}<\sqrt{\frac{1}{\beta^{2} a_{\gamma}^{2}}} \Rightarrow \overline{\alpha_{0}}>1
$$

so we just need to prove that

$$
\underline{\alpha}_{0} \equiv \frac{(T-\tau)+\beta a_{\gamma} \sqrt{\frac{T-\tau}{2 \beta}}}{2+\left(-\beta a_{\gamma}\right)\left(\frac{2(T-2)}{T}+\frac{T-4}{T} \sqrt{\frac{T-\tau}{2 \beta}}\right)}<1
$$

Rearranging gives

$$
\frac{T}{2}<\frac{(T-2)}{T-\tau-2}\left(-\beta a_{\gamma}\right)\left(1+\sqrt{\frac{T-\tau}{2 \beta}}\right)=\left(\frac{-\beta a_{\gamma}}{1-\beta}\right)\left(1+\sqrt{\frac{T-\tau}{2 \beta}}\right)
$$

Setting $\Delta \equiv \frac{T-\tau}{2}=\frac{(1-\beta) T+2 \beta}{2} \Leftrightarrow \frac{T}{2}=\frac{\Delta-\beta}{1-\beta}$, this rearranges to the inequality $a_{\gamma}<1-\sqrt{\frac{\Delta}{\beta}}$, which is implied by $a_{\gamma} \leq-1.7726 \Rightarrow \beta \geq .4172$ and $\Delta=2$, establishing (ii).

Now for (iii) and (iv): with $C_{u}$ as specified by (28), the equations in (11), (12) become

$u_{1}\left(a, \alpha_{a}\right)-\theta_{3}=K-\frac{T-2}{2} a-\sqrt{\frac{1-\alpha_{a}}{\alpha_{a}} \frac{1-\alpha_{0}}{\alpha_{0}} K^{2}+\frac{1-\alpha_{a}}{\alpha_{a}}(T-2) a\left(K-\frac{T}{4} a\right)}$
$v_{1}\left(a, \alpha_{0}\right)-\theta_{3}=\frac{2 K-\tau\left(a-a_{\gamma}\right)-\sqrt{\frac{T-\tau-2 \alpha_{0}}{2 \alpha_{0}}} \sqrt{\frac{2 \frac{T-\tau-2 \alpha_{0}}{\alpha_{0}} K^{2}+2 \tau\left(2 K\left(a-a_{\gamma}\right)-\frac{T}{2}\left(a-a_{\gamma}\right)^{2}\right)}{T-\tau}}}{\text { (46) }}$

We first prove (iii). To show that $v_{1}(\cdot)$ is real-valued, note that the term in square roots is decreasing in $a$ (derivative w.r.t. $a$ has the same sign as $2 K-T\left(a-a_{\gamma}\right)$, which is negative by $K<0$ and $a \geq a_{\gamma}$ ), so we just need to show that minimum value, which is at $a=0$, is non-negative: that is, we need

$$
0 \leq \frac{T-\tau-2 \alpha_{0}}{\tau \alpha_{0}} K^{2}-2 K a_{\gamma}-\frac{T}{2} a_{\gamma}^{2}
$$

The value of $K$ specified in (29) is precisely the negative root of this equation, so the above inequality holds by construction. For $u_{1}(\cdot)$ to be real-valued, we need

$$
0 \leq \frac{1-\alpha_{0}}{\alpha_{0}} K^{2}+(T-2) \min _{a \in[-2,0]} a\left(K-\frac{T}{4} a\right)
$$

The 2nd term is either minimized at $a=0$ (in which case the expression clearly holds), or at $a=-2$, in which case it holds (for $K<0$ ) iff

$$
K \leq \frac{\alpha_{0}(T-2)}{1-\alpha_{0}}\left(1-\sqrt{\frac{T-2 \alpha_{0}}{\alpha_{0}(T-2)}}\right)
$$


For this to be satisfied by the $K$-value in our construction, we need

$$
\begin{aligned}
& \frac{\alpha_{0} \tau\left(-a_{\gamma}\right)\left(1+\sqrt{\frac{\left(T-2 \alpha_{0}\right)(T-\tau)}{2 \tau \alpha_{0}}}\right)}{\left(T-\tau-2 \alpha_{0}\right)} \geq \frac{\alpha_{0}(T-2)}{1-\alpha_{0}}\left(\sqrt{\frac{T-2 \alpha_{0}}{\alpha_{0}(T-2)}}-1\right) \\
\Leftrightarrow & \frac{\left(-\beta a_{\gamma}\right)\left(1-\alpha_{0}\right)}{\left(T-\tau-2 \alpha_{0}\right)} \geq \frac{T\left(1-\alpha_{0}\right)}{\left(\sqrt{\alpha_{0}(T-2)\left(T-2 \alpha_{0}\right)}\left(1+\sqrt{\frac{T-\tau}{2 \beta}}\right)+\left(T-2 \alpha_{0}\right) \sqrt{\frac{T-\tau}{2 \beta}}+\alpha_{0}(T-2)\right)}
\end{aligned}
$$

(2nd line obtained from 1st by (i) replacing $\tau$ with $\beta(T-2)$ in the LHS numerator; (ii) multiplying both sides by $\frac{1-\alpha_{0}}{\alpha_{0}(T-2)\left(1+\sqrt{\frac{\left(T-2 \alpha_{0}\right)(T-\tau)}{2 \tau \alpha_{0}}}\right)}$; and finally (iii) on the resulting RHS, multiplying both numerator and denominator by $\left.\sqrt{\alpha_{0}(T-2)}\left(\sqrt{T-2 \alpha_{0}}+\sqrt{\alpha_{0}(T-2)}\right)\right)$. Note that $\sqrt{\alpha_{0}(T-2)\left(T-2 \alpha_{0}\right)} \geq \alpha_{0}(T-2)$, so the RHS is at most

$$
\frac{T\left(1-\alpha_{0}\right)}{\left(\alpha_{0}(T-2)\left(1+\sqrt{\frac{T-\tau}{2 \beta}}\right)+\left(T-2 \alpha_{0}\right) \sqrt{\frac{T-\tau}{2 \beta}}+\alpha_{0}(T-2)\right)}=\frac{T\left(1-\alpha_{0}\right)}{\left(\left(T-2 \alpha_{0}+\alpha_{0}(T-2)\right) \sqrt{\frac{T-\tau}{2 \beta}}+2 \alpha_{0}(T-2)\right)}
$$

Therefore, to prove the desired inequality, it is sufficient to show that the following holds:

$$
\begin{aligned}
\frac{\left(-\beta a_{\gamma}\right)}{\left(T-\tau-2 \alpha_{0}\right)} & \geq \frac{T}{\left(\left(T-2 \alpha_{0}+\alpha_{0}(T-2)\right) \sqrt{\frac{T-\tau}{2 \beta}}+2 \alpha_{0}(T-2)\right)} \\
& \Leftrightarrow \alpha_{0} \geq \frac{(T-\tau)+\beta a_{\gamma} \sqrt{\frac{T-\tau}{2 \beta}}}{2+\left(-\beta a_{\gamma}\right)\left(\frac{2(T-2)}{T}+\frac{T-4}{T} \sqrt{\frac{T-\tau}{2 \beta}}\right)}
\end{aligned}
$$

The RHS is precisely the value $\underline{\alpha_{0}}$ defined in the Lemma, so $\alpha_{0} \geq \underline{\alpha_{0}}$ is sufficient to guarantee that $u_{t}(\cdot)$ is real-valued. This completes the proof of (iii).

Finally, to prove (iv): by (45) and (46) we have $u_{1}\left(0, \alpha_{0}\right)=K-\frac{1-\alpha_{0}}{\alpha_{0}} \sqrt{K^{2}}=K\left(1+\frac{1-\alpha_{0}}{\alpha_{0}}\right)=$ $\frac{K}{\alpha_{0}}$, and $u_{1}(-2,1)=K+T-2$. So, if we set $\alpha(0)=\alpha_{0}$ and $\alpha(-2)=1$, then $\left[\frac{K}{\alpha_{0}}, K+T-2\right] \subseteq$ $\left[u_{1}^{\min }, u_{1}^{\max }\right]$. On the other hand,

$$
\begin{aligned}
& \frac{T-\tau}{\tau} \frac{d v_{1}\left(a, \alpha_{0}\right)}{d a}=-1+\frac{\left(\frac{T}{2}\left(a-a_{\gamma}\right)-K\right)}{\sqrt{K^{2}+\frac{\tau \alpha_{0}}{T-\tau-2 \alpha_{0}}\left(2 K\left(a-a_{\gamma}\right)-\frac{T}{2}\left(a-a_{\gamma}\right)^{2}\right)}} \\
& \frac{T-\tau}{2} \frac{d^{2} v_{1}\left(a, \alpha_{0}\right)}{d a^{2}}=\frac{\frac{T}{2}{\left.\sqrt{K^{2}+\frac{\alpha_{0}}{T-\tau-2 \alpha_{0}} \tau\left(2 K\left(a-a_{\gamma}\right)-\frac{T}{2}\left(a-a_{\gamma}\right)^{2}\right.}\right)^{2}+\frac{\tau \alpha_{0}}{T-\tau-2 \alpha_{0}}\left(\frac{T}{2}\left(a-a_{\gamma}\right)-K\right)^{2}}_{\sqrt{K^{2}+\frac{\tau \alpha_{0}}{T-\tau-2 \alpha_{0}}\left(2 K\left(a-a_{\gamma}\right)-\frac{T}{2}\left(a-a_{\gamma}\right)^{2}\right.}}^{3}}{3}
\end{aligned}
$$

Clearly the second expression is positive, so we have that

$$
a \geq a_{\gamma} \Rightarrow \frac{T-\tau}{\tau} \frac{d v_{1}\left(a, \alpha_{0}\right)}{d a} \geq\left.\frac{T-\tau}{\tau} \frac{d v_{1}\left(a, \alpha_{0}\right)}{d a}\right|_{a=a_{\gamma}}=-1+\frac{(-K)}{\sqrt{K^{2}}}=0
$$


That is, $v_{1}(\cdot)$ is strictly increasing on $\left[a_{\gamma}, 0\right]$, with

$$
\begin{aligned}
v_{1}^{\min }-\theta_{3} & =v_{1}\left(a_{\gamma}, \alpha_{0}\right)-\theta_{3}=\frac{2 K-\sqrt{\frac{T-\tau-2 \alpha_{0}}{2 \alpha_{0}}} \sqrt{2 \frac{T-\tau-2 \alpha_{0}}{\alpha_{0}} K^{2}}}{T-\tau}=\frac{2 K\left(1+\frac{T-\tau-2 \alpha_{0}}{2 \alpha_{0}}\right)}{T-\tau}=\frac{K}{\alpha_{0}} \\
v_{1}^{\max }-\theta_{3} & =v_{1}\left(0, \alpha_{0}\right)-\theta_{3}=\frac{2 K+\tau a_{\gamma}-\sqrt{\frac{T-\tau-2 \alpha_{0}}{2 \alpha_{0}}} \sqrt{2 \tau\left(\frac{T-\tau-2 \alpha_{0}}{\tau \alpha_{0}} K^{2}-2 K a_{\gamma}-\frac{T}{2} a_{\gamma}^{2}\right)}}{T-\tau} \\
& =\frac{2 K+\tau a_{\gamma}}{T-\tau}
\end{aligned}
$$

So we immediately have $v_{1}^{\min }=u_{1}\left(0, \alpha_{0}\right)$. To show that $v_{1}^{\max } \leq u_{1}(-2,1)$, we need to show that

$$
K+T-2 \geq \frac{2 K+\tau a_{\gamma}}{T-\tau} \Leftrightarrow \frac{(T-\tau-2)}{(T-2)} K>\frac{\tau a_{\gamma}}{T-2}-(T-\tau)
$$

Substituting in our value $K=\frac{a_{\gamma}\left(\tau \alpha_{0}+\sqrt{\frac{\left(T-2 \alpha_{0}\right)\left(\tau \alpha_{0}\right)(T-\tau)}{2}}\right)}{\left(T-\tau-2 \alpha_{0}\right)}$, using $\tau=\beta(T-2)$, and dividing by $a_{\gamma}$ (which flips the inequality), this becomes

$\frac{a_{\gamma}\left(\tau \alpha_{0}+\sqrt{\frac{\left(T-2 \alpha_{0}\right)\left(\tau \alpha_{0}\right)(T-\tau)}{2}}\right)}{\left(T-\tau-2 \alpha_{0}\right)}>\frac{\beta a_{\gamma}-(T-\tau)}{1-\beta} \Leftrightarrow \frac{(T-2)\left(\beta \alpha_{0}+\sqrt{\frac{\left(T-2 \alpha_{0}\right)\left(\beta \alpha_{0}\right)(T-\tau)}{2(T-2)}}\right)}{\left(T-\tau-2 \alpha_{0}\right)}<\frac{\beta+\frac{(T-\tau)}{\left(-a_{\gamma}\right)}}{1-\beta}$

For this, it is sufficient to prove the following (which replaces the 2nd numerator term on the LHS with its maximum value (at $\left.\alpha_{0}=1\right)$ ):

$$
\frac{(T-2)\left(\beta \alpha_{0}+\sqrt{\frac{\beta(T-\tau)}{2}}\right)}{\left(T-\tau-2 \alpha_{0}\right)}<\frac{\beta+\frac{(T-\tau)}{-a_{\gamma}}}{1-\beta}
$$

Multiplying both sides by $(1-\beta)\left(T-\tau-2 \alpha_{0}\right)$, this rearranges as

$$
(T-\tau-2) \sqrt{\frac{\beta(T-\tau)}{2}}-\left(T-\tau-2 \alpha_{0}\right) \frac{(T-\tau)}{-a_{\gamma}}<\beta(T-\tau)\left(1-\alpha_{0}\right)
$$

Replacing the LHS with the upper bound at $\alpha_{0}=1$, and dividing through by $\beta(T-\tau)$, we obtain the following sufficient condition:

$$
\alpha_{0} \leq 1-(T-\tau-2)\left(\sqrt{\frac{1}{2 \beta(T-\tau)}}-\frac{1}{-\beta a_{\gamma}}\right)
$$

The RHS is precisely the value $\overline{\alpha_{0}}$ defined in the Lemma: that is, $\alpha_{0} \leq \overline{\alpha_{0}}$ implies that $\left[v_{1}^{\min }, v_{1}^{\max }\right] \subseteq$ $\left[u_{1}\left(0, \alpha_{0}\right), u_{1}(-2,1)\right]$, which, by continuity, implies that for every $a \in\left[a_{\gamma}, 0\right] \Leftrightarrow v_{1}(a) \in\left[v_{1}^{\min }, v_{1}^{\max }\right]$, there exists $\widehat{a}(a) \in[-2,0]$ and $\alpha_{a} \geq \alpha_{0}$ s.t. $v_{1}\left(a, \alpha_{0}\right)=u_{1}\left(\widehat{a}(a), \alpha_{a}\right)$. This proves (iv). 


\section{E Appendix E: Derivations}

In this section, we explain how the functions and parameters in our fully revealing construction were chosen.

\section{E.1 For the Expert:}

Suppose we wanted an equilibrium in which each type $\theta \in\left[0, \theta_{1}\right]$ pools with a type $g(\theta) \in\left[\theta_{2}, \theta_{3}\right]$, to recommend an action $u_{1}(\theta)$ in period $1, u_{2}(\theta)$ in period 2 , and then reveal the truth at time $T-2$. The disutility to type $\theta$ from following the strategies prescribed for types $\theta^{\prime}, g\left(\theta^{\prime}\right)$ is then

$$
\begin{aligned}
D_{u}\left(\theta^{\prime} \mid \theta\right) & =\left(u_{1}\left(\theta^{\prime}\right)-\theta-1\right)^{2}+\left(u_{2}\left(\theta^{\prime}\right)-\theta-1\right)^{2}+(T-2)\left(\theta^{\prime}-\theta-1\right)^{2} \\
D_{u}\left(g\left(\theta^{\prime}\right) \mid \theta\right) & =\left(u_{1}\left(\theta^{\prime}\right)-g(\theta)-1\right)^{2}+\left(u_{2}\left(\theta^{\prime}\right)-g(\theta)-1\right)^{2}+(T-2)\left(g\left(\theta^{\prime}\right)-g(\theta)-1\right)^{2}
\end{aligned}
$$

In order for this to be an equilibrium, it must be that $D_{u}\left(\theta^{\prime} \mid \theta\right)$ reaches a minimum over $\left[0, \theta_{1}\right]$ at $\theta^{\prime}=\theta$ (so that type $\theta$ earns a lower disutility by telling the truth than by mimicking any other type $\theta^{\prime}$ in the interval $\left.\left[0, \theta_{1}\right]\right)$, and that $D_{u}\left(g\left(\theta^{\prime}\right) \mid g(\theta)\right)$ reaches a minimum at $g\left(\theta^{\prime}\right)=g(\theta)$. We can do this by simply choosing functions that satisfy the corresponding first- and second-order conditions: beginning with the F.O.C.'s, we need

$$
\begin{aligned}
0 & \left.=\left.\frac{1}{2} \frac{d}{d \theta^{\prime}} D_{u}\left(\theta^{\prime} \mid \theta\right)\right|_{\theta^{\prime}=\theta}=u_{1}^{\prime}(\theta)\left(u_{1}(\theta)-\theta-1\right)\right)+u_{2}^{\prime}(\theta)\left(u_{2}(\theta)-\theta-1\right)-(T-2) \\
0 & =\left.\frac{1}{2} \frac{d}{d \theta^{\prime}} D_{u}\left(\theta^{\prime} \mid \theta\right)\right|_{\theta^{\prime}=\theta}=u_{1}^{\prime}(\theta)\left(u_{1}(\theta)-g(\theta)-1\right)+u_{2}^{\prime}(\theta)\left(u_{2}(\theta)-\theta-1\right)-(T-2) g^{\prime}(\theta)(49)
\end{aligned}
$$

Subtracting the 2nd expression from the 1st, we get

$$
\left(u_{1}^{\prime}(\theta)+u_{2}^{\prime}(\theta)\right)(g(\theta)-\theta)=(T-2)\left(1-g^{\prime}(\theta)\right)
$$

If we define $a(\theta) \equiv \ln \frac{g(\theta)-\theta}{g\left(\theta_{1}\right)-\theta_{1}}$, so that $a^{\prime}(\theta)=\frac{g^{\prime}(\theta)-1}{g(\theta)-\theta}$, this becomes

$$
u_{1}^{\prime}(\theta)+u_{2}^{\prime}(\theta)=-(T-2) a^{\prime}(\theta) \Rightarrow u_{1}(\theta)+u_{2}(\theta)=k_{u}-(T-2) a(\theta), k_{u} \text { a constant }
$$

Now: the disutility from telling the truth is

$$
\begin{aligned}
D_{u}(\theta \mid \theta) & \equiv D_{u}(\theta)=\left(u_{1}(\theta)-\theta-1\right)^{2}+\left(u_{2}(\theta)-\theta-1\right)^{2}+(T-2) \\
& \Rightarrow \frac{D_{u}^{\prime}(\theta)}{2}=\left(u_{1}^{\prime}(\theta)-1\right)\left(u_{1}(\theta)-\theta-1\right)+u_{2}^{\prime}(\theta)\left(u_{2}(\theta)-\theta-1\right)
\end{aligned}
$$

Substituting (48) into this expression, we get

$$
\begin{aligned}
\frac{D_{u}^{\prime}(\theta)}{2} & =T-2-\left(u_{1}(\theta)+u_{2}(\theta)\right)+2(\theta+1) \\
& =T+2 \theta-k_{u}+(T-2) a(\theta)(\text { by }(50))
\end{aligned}
$$

Integrating w.r.t. $\theta$, we get

$$
D_{u}(\theta)=D_{u}(0)+2 \theta\left(T+\theta-k_{u}\right)-2(T-2) \int_{0}^{\theta}\left(a\left(\theta^{\prime}\right)\right) d \theta^{\prime}
$$


Setting $u_{1}(0) \equiv u_{0}$ and using expression (50) to obtain $u_{2}(0)=k_{u}-(T-2) a(0)-u_{1}(0)=k_{u}-u_{0}$, this becomes

$$
\begin{aligned}
D_{u}(\theta) & =\underbrace{\left(u_{0}-1\right)^{2}+\left(k_{u}-u_{0}-1\right)^{2}+(T-2)}_{D_{u}(0)}+2 \theta\left(T+\theta-k_{u}\right)+2(T-2) \int_{0}^{\theta} a\left(\theta^{\prime}\right) d \theta^{\prime} \\
& =2\left(\frac{k_{u}}{2}-u_{0}\right)^{2}+2\left(\frac{k_{u}}{2}-\theta-1\right)^{2}+2 \theta(T-2)+2(T-2) \int_{0}^{\theta} a\left(\theta^{\prime}\right) d \theta^{\prime}+(T-2)
\end{aligned}
$$

It will be convenient to change variables: rather than describing $g$ as a function from $\left[0, \theta_{1}\right] \rightarrow$ $\left[\theta_{2}, \theta_{3}\right]$, and using $a(\theta) \equiv \ln \frac{g(\theta)-\theta}{g(0)-0}$, we "flip" variables, describing each type $\theta \in\left[0, \theta_{1}\right]$ as a parametric function $x(a)$ of the variable $a$, and each type in $\left[\theta_{2}, \theta_{3}\right]$ as a parametric function $g(a)$ of the variable $a$, where $a$ takes on all values between 0 and $a_{1}=\ln \frac{g\left(\theta_{1}\right)-\theta_{1}}{g(0)-0}$, and $g(a), x(a)$ hold the relationship $(g(a)-x(a))=(g(0)-x(0)) e^{a}$. With this, rewriting $\int_{0}^{\theta} a\left(\theta^{\prime}\right) d \theta^{\prime}$ as $\int_{0}^{a} s x^{\prime}(s) d s$, and noting that $\theta=\int_{0}^{a} x^{\prime}(s) d s$, our above disutility expression for type $\theta=x(a)$ becomes

$$
D_{u}(x(a))=2\left(\frac{k_{u}}{2}-x(a)-1\right)^{2}+2(T-2) \int_{0}^{a}(s+1) x^{\prime}(s) d s+(T-2)+2(T-2) C_{u}
$$

where $C_{u} \equiv \frac{\left(\frac{k_{u}}{2}-u_{0}\right)^{2}}{T-2}$ may be any non-negative constant. Setting this equal to type $x(a)^{\prime} s$ truthtelling disutility (evaluate (51) at $\theta=x(a)$ ), using $u_{2}(a)=k-(T-2) a-u_{1}(a)$ (from (50)), and solving for $u_{1}(a), u_{2}(a)$, we obtain

$$
\begin{aligned}
u_{1}(a) & =\frac{k_{u}}{2}-\frac{T-2}{2} a-\sqrt{T-2} \sqrt{\left.C_{u}+\frac{k_{u}}{2} a-a(x(a)+1)-\frac{T-2}{4} a^{2}+\int_{0}^{a}(s+1) x^{\prime}(s) d \$ 53\right)} \\
& =\frac{k_{u}}{2}-\frac{T-2}{2} a-\sqrt{T-2} \sqrt{C_{u}+\frac{k_{u}}{2} a-a(g(a)+1)-\frac{T-2}{4} a^{2}+\int_{0}^{a}(s+1) g^{\prime}(s) d s}
\end{aligned}
$$

with $u_{2}(a)=k_{u}-(T-2) a-u_{1}(a)$. Evaluating this at $x(a)=\theta_{3}+a-\theta_{3} e^{a}$ and $\frac{k_{u}}{2}=K+\theta_{3}$ gives precisely our expression $u_{1}\left(a, \alpha_{a}\right)$ in (11) evaluated at $\alpha_{a}=\frac{1}{2}$; the expressions in (11), (12) were "rescaled" (via the coefficients on the square roots) such that both disutility and average actions are independent of $\alpha_{a}$.

Now, for our S.O.C.'s: differentiating (47) w.r.t. $\theta^{\prime}$ gives

$$
\begin{aligned}
\frac{1}{2} \frac{d D_{u}\left(\theta^{\prime} \mid \theta\right)}{d \theta^{\prime}}= & \underbrace{u_{1}^{\prime}\left(\theta^{\prime}\right)\left(u_{1}\left(\theta^{\prime}\right)-\theta^{\prime}-1\right)+u_{2}^{\prime}\left(\theta^{\prime}\right)\left(u_{2}\left(\theta^{\prime}\right)-\theta^{\prime}-1\right)-(T-2)}_{=0 \text { by }(48)} \\
& +\left(\theta^{\prime}-\theta\right) \underbrace{\left(u_{1}^{\prime}\left(\theta^{\prime}\right)+u_{2}^{\prime}\left(\theta^{\prime}\right)+T-2\right)}_{=\frac{d}{d \theta^{\prime}}\left(u_{1}\left(\theta^{\prime}\right)+u_{2}\left(\theta^{\prime}\right)+(T-2) \theta^{\prime}\right)}
\end{aligned}
$$

This implies that a sufficient condition for truth-telling to indeed yield a minimum on disutility is that the average action induced by each type $\theta, u_{1}(\theta)+u_{2}(\theta)+(T-2) \theta$, be increasing: in this case, $\frac{d D_{u}\left(\theta^{\prime} \mid \theta\right)}{d \theta^{\prime}}$ is positive for any $\theta^{\prime}>\theta$ (as type $\theta$ contemplates mimicking types $\theta^{\prime}$ further above him, disutility increases, making him worse off), and negative for $\theta^{\prime}<\theta$ (as he moves further below the truth, disutility increases, also making him worse off), but zero at $\theta^{\prime}=\theta$ : thus, telling the truth is better than mimicking any other type in the interval.

To sum up, this has shown that given arbitrary interval endpoints $\theta_{1}, \theta_{2}, \theta_{3}$, functions $x$ : 
$\left[a_{1}, 0\right] \rightarrow\left[0, \theta_{1}\right]$ and $g:\left[a_{1}, 0\right] \rightarrow\left[\theta_{2}, \theta_{3}\right]$, and with $a_{1} \equiv \ln \frac{g\left(a_{1}\right)-x\left(a_{1}\right)}{g(0)-x(0)}=\ln \frac{\theta_{2}-\theta_{1}}{\theta_{3}-0}$ and $g(a)-x(a)=$ $\theta_{3} e^{a}$, if we want an equilibrium in which types $x(a), g(a)$ recommend $u_{1}(a)$ for one period, then $u_{2}(a)$ for one period, then separate and reveal the truth, then truth-telling satisfies the F.O.C. for disutility minimization iff $u_{1}, u_{2}$ are as specified by (53) and $u_{2}(a)=k_{u}-(T-2) a-u_{1}(a)$. If we additionally impose the requirement that average action be increasing in type, then we satisfy also the S.O.C.'s; this requires that each of $x^{\prime}(a), g^{\prime}(a)$ is either negative or $\geq 1$. Analogously, for arbitrary functions $z:\left[a_{\gamma}, 0\right] \rightarrow\left[\theta_{1}, \theta_{2}\right], h:\left[a_{\gamma}, 0\right] \rightarrow\left[\theta_{3}, \theta_{4}\right]$, with $a_{\gamma}=\ln \frac{h\left(a_{\gamma}\right)-z\left(a_{\gamma}\right)}{h(0)-z(0)}=\frac{\theta_{3}-\theta_{2}}{\theta_{4}-\theta_{1}}$ and $h(a)-z(a)=\left(\theta_{4}-\theta_{1}\right) e^{a}$, if we want an equilibrium in which types $z(a), h(a)$ recommend $v_{1}(a)$ for $\frac{T-\tau}{2}$ periods, then $v_{2}(a)$ for $\frac{T-\tau}{2}$ periods, then separate and reveal the truth, the F.O.C.'s for truth-telling to minimize disutility yield the following equations:

$$
v_{1}(a)=\frac{k_{v}}{2}-\frac{\tau}{T-\tau} a-\sqrt{\frac{2 \tau}{T-\tau}} \sqrt{C_{v}+\frac{k_{v}}{2} a-a(h(a)+1)-\frac{\tau}{2(T-\tau)} a^{2}+\int_{0}^{a}(s+1) h^{\prime}(s) d s}
$$

with $v_{2}(a)=k_{v}-\frac{2 \tau}{T-\tau} a-v_{1}(a), k_{v}$ and $C_{v}$ constants. And the S.O.C.'s, guaranteeing that truthtelling indeed yields a disutility minimum over the interval, reduce to the requirement that each of $z^{\prime}(a), h^{\prime}(a)$ is either negative or $\geq 1$. The proof that no expert type wishes to deviate after the initial recommendation follows almost trivially from the prescribed strategies.

It remains to show that no expert type wishes to mimic the initial recommendation of any type from any other interval. This reduces to the additional requirements that at each endpoint $\theta_{i} \in\left\{\theta_{1}, \theta_{2}, \theta_{3}\right\}$, the average action is non-decreasing at $\theta_{i}$ (if discontinuous), and type $\theta_{i}$ is indifferent between the two sequences that he can induce. Our construction chooses the specific parameterizations $g(a)=\theta_{3}+a$ and $h(a)=\theta_{4}+a$, with $x(a)=g(a)-\theta_{3} e^{a}, z(a)=h(a)-\left(\theta_{4}-\theta_{1}\right) e^{a}$. Then we have $x^{\prime}(a)=1-\theta_{3} e^{a} \leq 1-\theta_{3} e^{-2}, z^{\prime}(a)=1-2 e^{a-a_{\gamma}} \leq-1$, and $g^{\prime}(a)=h^{\prime}(a)=1$, which clearly satisfy the S.O.C.'s (provided that $\theta_{3} e^{-2} \geq 2$; we in fact will restrict to $\theta_{3} e^{-2} \geq 8$ ). With this, the expressions in (53), (54) become (with $K \equiv \frac{k_{u}}{2}-\theta_{3}$ )

$$
\begin{aligned}
& u_{1}(a)=K+\theta_{3}-\frac{T-2}{2} a-\sqrt{T-2} \sqrt{C_{u}+K a-\frac{T}{4} a^{2}} \\
& v_{1}(a)=\frac{k_{v}}{2}-\frac{\tau}{T-\tau} a-\sqrt{\frac{2 \tau}{T-\tau}} \sqrt{C_{v}+\left(\frac{k_{v}}{2}-\theta_{4}\right) a-\frac{T}{2(T-\tau)} a^{2}}
\end{aligned}
$$

We chose $a_{1}=-2$ and $g^{\prime}(a)=1$ because this is in fact the only way that the indifference constraint at $\theta_{2}$ can hold simultaneously with both the indifference constraint at $\theta_{3}$, and the increasingaverage-action requirement at $\theta_{2}$. We chose $h^{\prime}(a)=1$ just for simplicity. With it, the remaining increasing-average-action constraints do not bind, and the indifference conditions reduce to the following requirements on the relationships between $k_{v}, C_{v}, \tau$ (parameters from the $v_{t}$-functions) and $k_{u}, C_{u}, T$ (parameters from the $u_{t}$-functions):

$$
\begin{aligned}
& \frac{k_{v}}{2}-\theta_{3}=\frac{2 K+\tau a_{\gamma}}{T-\tau} \\
& C_{v}=\frac{(T-2) C_{u}}{\tau}+\frac{\frac{T-\tau-2}{\tau} K^{2}-2 K a_{\gamma}-\frac{T}{2} a_{\gamma}^{2}}{(T-\tau)} \\
& \frac{\tau}{T-2}=\beta \equiv \frac{\left(\theta_{2}-\theta_{1}\right)\left(\theta_{2}-\theta_{1}-2\right)}{\left(\theta_{4}-\theta_{1}\right)\left(\theta_{4}-\theta_{1}-2\right)}
\end{aligned}
$$

With this, the expressions in (55), (56) simplify exactly to the expressions for $u_{1}\left(a, \alpha_{a}\right)$ in (11) and $v_{1}\left(a, \alpha_{0}\right)$ in (13), at $\alpha_{a}=\frac{1}{2}, \alpha_{0}=\frac{T-\tau}{4}$; in Proposition D3, we then rescaled (11),(13) for other values of $\alpha_{a}, \alpha_{0}$ in such a way that incentives are not affected. 


\section{E.2 For the DM:}

Suppose the DM receives the recommendation $u_{1}\left(a, \alpha_{a}\right)$ in period 1. If he assigns probabilities $\left(p_{a}, 1-p_{a}\right)$ to types $x(a), g(a)$, then his disutility from following all recommendations is

$$
\begin{aligned}
& p_{a}\left(2 \alpha_{a}\left(u_{1}\left(a, \alpha_{a}\right)-x(a)\right)^{2}+2\left(1-\alpha_{a}\right)\left(u_{2}\left(a, \alpha_{a}\right)-x(a)\right)^{2}\right) \\
& +\left(1-p_{a}\right)\left(2 \alpha_{a}\left(u_{1}\left(a, \alpha_{a}\right)-g(a)\right)^{2}+2\left(1-\alpha_{a}\right)\left(u_{2}\left(a, \alpha_{a}\right)-g(a)\right)^{2}\right)
\end{aligned}
$$

Substituting in the expression for $u_{1}\left(a, \alpha_{a}\right), u_{2}\left(a, \alpha_{a}\right)$ from $(11),(12)$, and expanding, this becomes

$2 p_{a}\left(K+\theta_{3}-\frac{T-2}{2} a-x(a)\right)^{2}+2\left(1-p_{a}\right)\left(K+\theta_{3}-\frac{T-2}{2} a-g(a)\right)^{2}+2(T-2) C_{u}+2(T-2) a\left(K-\frac{T}{4} a\right)$

The best possible deviation is to choose the myopically optimal action $p_{a} x(a)+\left(1-p_{a}\right) g(a)$ in all $T$ periods, resulting in disutility

$$
\begin{aligned}
& \left.T p_{a}\left(p_{a} x(a)+\left(1-p_{a}\right) g(a)-x(a)\right)^{2}+T\left(1-p_{a}\right)\left(p_{a} x(a)+1-p_{a}\right) g(a)-g(a)\right)^{2} \\
= & T p_{a}\left(1-p_{a}\right)(g(a)-x(a))^{2}
\end{aligned}
$$

Therefore, incentive compatibility of our strategies for the DM refers that the following expression (the gain to deviating at $\{x(a), g(a)\}$ ) be weakly negative for all $a \in[-2,0]$ :

$$
\begin{aligned}
& 2 p_{a}\left(K+\theta_{3}-\frac{T-2}{2} a-x(a)\right)^{2}+2\left(1-p_{a}\right)\left(K+\theta_{3}-\frac{T-2}{2} a-g(a)\right)^{2} \\
& +2(T-2) C_{u}+2(T-2) a\left(K-\frac{T}{4} a\right)-T p_{a}\left(1-p_{a}\right)(g(a)-x(a))^{2}
\end{aligned}
$$

Substituting in $g(a)=\theta_{3}+a, x(a)=\theta_{3}+a-\theta_{3} e^{a}$ and solving for $K$, we obtain that the DM's gain to deviating at information set $\{x(a), g(a)\}$ is negative if and only if

$$
\begin{aligned}
K & \in\left[a-p_{a} \theta_{3} e^{a}-\sqrt{T-2} \Delta(a), a-p_{a} \theta_{3} e^{a}+\sqrt{T-2} \Delta(a)\right] \\
\text { where } \Delta(a) & \equiv \sqrt{\frac{1}{2} p_{a}\left(1-p_{a}\right)\left(\theta_{3} e^{a}\right)^{2}+p_{a}\left(\theta_{3} a e^{a}\right)-\frac{a^{2}}{2}-C_{u}}
\end{aligned}
$$

For there to exist a value of $K$ which satisfies this expression, we need $\Delta(a)$ to be real-valued, i.e. the term in square roots must be positive; at $a=-2$, this holds iff

$$
p_{a} \in\left[\frac{\theta_{3} e^{-2}-4}{2\left(\theta_{3} e^{-2}\right)}-\frac{1}{2} \sqrt{\frac{\theta_{3} e^{-2}-8}{\theta_{3} e^{-2}}}, \frac{\theta_{3} e^{-2}-4}{2\left(\theta_{3} e^{-2}\right)}+\frac{1}{2} \sqrt{\frac{\theta_{3} e^{-2}-8}{\theta_{3} e^{-2}}}\right]
$$

which in turn is possible (for some belief system) to satisfy only if $\theta_{3} \geq 8 e^{2}$. For our construction, with $g^{\prime}=h^{\prime}=1$, this corresponds to

$$
a_{\gamma}=\ln \frac{\theta_{3}-\theta_{2}}{\theta_{4}-\theta_{1}}=\ln \frac{2}{-a_{\gamma}+2+\theta_{3} e^{-2}} \leq-1.7726 \Leftrightarrow b \leq \frac{1}{60.885}
$$

That is, it is possible to satisfy the DM IC constraints in a straightforward manner only if the bias satisfies $b \leq \frac{1}{60.885}$, which is why our construction specifies $b<\frac{1}{61}$. 
Similarly, if the DM receives the recommendation $v_{1}(a)$ in period 1 and assigns probabilities $q_{a}, 1-q_{a}$ to types $z(a), h(a)$, his maximum gain to deviating is

$$
\begin{aligned}
& q_{a}\left(2 \alpha_{0}\left(v_{1}\left(a, \alpha_{0}\right)-z(a)\right)^{2}+\left(T-\tau-2 \alpha_{0}\right)\left(v_{2}\left(a, \alpha_{0}\right)-z(a)\right)^{2}\right) \\
& +\left(1-q_{a}\right)\left(2 \alpha_{0}\left(v_{1}\left(a, \alpha_{0}\right)-h(a)\right)^{2}+\left(T-\tau-2 \alpha_{0}\right)\left(v_{2}\left(a, \alpha_{0}\right)-h(a)\right)^{2}\right)-T q_{a}\left(1-q_{a}\right)(h(a)-z(a))^{2}
\end{aligned}
$$

Recalling that the expressions in $(13),(14)$ were scaled to make the above expression independent of $\alpha_{0}$, we can without loss of generality set $\alpha_{0}=1$, in which case $v_{1}$ is given by (56), and $v_{2}=$ $k_{v}-\frac{2 \tau}{T-\tau} a-v_{1}$; substituting into the above expression for the DM's gain to deviating, we obtain

$$
\begin{aligned}
& (T-\tau)\left(q_{a}\left(\frac{k_{v}}{2}-\frac{\tau}{T-\tau} a-z\right)^{2}+\left(1-q_{a}\right)\left(\frac{k_{v}}{2}-\frac{\tau}{T-\tau} a-h\right)^{2}\right)+2 \tau C_{v} \\
& +2 \tau a\left(\frac{k_{v}}{2}-\theta_{4}-\frac{T}{2(T-\tau)} a\right)-T q_{a}\left(1-q_{a}\right)(h-z)^{2}
\end{aligned}
$$

Setting $h(a)=\theta_{4}+a, z(a)=\theta_{4}+a-\left(\theta_{4}-\theta_{1}\right) e^{a}=\theta_{4}+a-2 e^{a-a_{\gamma}}$ and solving for $\frac{k_{v}}{2}$, we obtain that the DM's gain to deviating at $\{z(a), h(a)\}$ is negative if and only if

$$
\begin{aligned}
\frac{k_{v}}{2} & \in\left[\theta_{4}+a-q_{a}\left(2 e^{a-a_{\gamma}}\right)-\sqrt{\frac{2 \tau}{T-\tau}} \widetilde{\Delta}(a), \theta_{4}+a-q_{a}\left(2 e^{a-a_{\gamma}}\right)+\sqrt{\frac{2 \tau}{T-\tau}} \widetilde{\Delta}(a)\right] \\
\text { where } \widetilde{\Delta}(a) & \equiv \sqrt{\frac{1}{2} q_{a}\left(1-q_{a}\right)\left(2 e^{a-a_{\gamma}}\right)^{2}+q_{a}\left(2 a e^{a-a_{\gamma}}\right)-\frac{a^{2}}{2}-C_{v}}
\end{aligned}
$$

This constraint by itself is problematic. To understand the difficulty, note that at $a=a_{\gamma}$, there exists a value of $k_{v}$ satisfying the above expression only if $\widetilde{\Delta}\left(a_{\gamma}\right)$ is real-valued, requiring

$$
2 q_{a}\left(1-q_{a}+a_{\gamma}\right)-\frac{a_{\gamma}^{2}}{2}-C_{v} \geq 0
$$

We showed in the previous paragraph that the IC constraints at information sets of the form $\{x(a), g(a)\}$ can only hold if $a_{\gamma} \leq-1.7726$, in this case, the first term in the above inequality is negative (since we need $q_{a} \geq 0$ and $1+a_{\gamma}<0$ ), the second term is clearly negative, and the third must be negative (i.e. we need $C_{v} \geq 0$ ) in order for the functions $v_{1}, v_{2}$ to be real-valued at $a=0$. Therefore, if the DM finds it optimal to follow all recommendations sent by pairs $\{x(a), g(a)\}$, then he necessarily will have an incentive to deviate if his information set contains only types $\left\{z\left(a_{\gamma}\right), h\left(a_{\gamma}\right)\right\}=\left\{\theta_{2}, \theta_{3}\right\}$. To solve this problem, we will "bunch" pairs - scaling our action functions such that whenever the DM would have an incentive to deviate after a recommendation $v_{1}$ sent by a pair $\{z(a), h(a)\}$, he believes that the recommendation is also sent (for the same length of time) by a pair $\left\{x\left(a^{\prime}\right), g\left(a^{\prime}\right)\right\}$, and such that the expected benefit to following the recommendation (likelihood that it was sent by the pair $\left(x\left(a^{\prime}\right), g\left(a^{\prime}\right)\right)$, times the gain in this case) exceeds the cost (which is the likelihood that it was sent by pair $(z(a), h(a))$, times the cost in this case).

\section{E.2.1 DM Beliefs}

Our incentive constraints for the DM were specified in terms of arbitrary probabilities $p_{a}, q_{a}$, which in turn depend both on his prior $F$, and on the precise details of our construction. We assume that the DM is Bayesian: if he believes that the expert's first-period messages are given by a function 
$M:[0,1] \rightarrow \mathbb{R}$, with the property that

$$
M(x)=M(p(x))
$$

for all $x$ in some interval $[\underline{x}, \bar{x}]$ and $p:[\underline{x}, \bar{x}] \rightarrow[0,1] \backslash[\underline{x}, \bar{x}]$ some continuous differentiable function (i.e., types $x$ and $p(x)$ are "partners" who follow the same messaging strategy), then, after receiving the message $m=M(x)=M(p(x))$, the DM's beliefs satisfy

$$
\frac{\operatorname{Pr}(x \mid m)}{\operatorname{Pr}(p(x) \mid m)}=\lim _{\Delta \rightarrow 0} \frac{F(x+\Delta)-F(x-\Delta)}{F(p(x+\Delta))-F(p(x-\Delta))}={ }_{\text {if } F \text { has a density } f)} \frac{f(x)}{f(p(x))}\left|\frac{1}{p^{\prime}(x)}\right|
$$

This says that the likelihood of type $x$ relative to $p(x)$ is equal to the unconditional likelihood ratio (determined by the prior $F$ ), times a term which depends on the shape of the $p$-function, in particular due to its influence on the size of the interval of $p$-types compared to their partner interval, $[\underline{x}, \bar{x}]$. For our construction, (61) becomes:

- after a message (or message sequence) sent by types $\{x(a), g(a)\}, a \in[-2,0]$ :

$$
\frac{p_{a}}{1-p_{a}} \equiv \frac{\operatorname{Pr}(x(a))}{\operatorname{Pr}(g(a))}=\frac{f(x(a))}{f(g(a))} \cdot\left|\frac{x^{\prime}(a)}{g^{\prime}(a)}\right|=\frac{f(x(a))}{f(g(a))}\left(\theta_{3} e^{a}-1\right)
$$

- after a message (or message sequence) sent by types $\{z(a), h(a)\}, a \in\left[a_{\gamma}, 0\right]$ :

$$
\frac{q_{a}}{1-q_{a}} \equiv \frac{\operatorname{Pr}(z(a)}{\operatorname{Pr}(h(a)}=\frac{f(z(a))}{f(h(a))} \cdot\left|\frac{z^{\prime}(a)}{h^{\prime}(a)}\right|=\frac{f(z(a))}{f(h(a))}\left(2 e^{a-a_{\gamma}}-1\right)
$$

- and, after a message sent by types $\{z(a), h(a), x(\widehat{a}(a)), g(\widehat{a}(a))\}$, with $u_{1}\left(\widehat{a}(a) \alpha_{\widehat{a}}\right)=v_{1}\left(a, \alpha_{0}\right)$ :

$$
\frac{\operatorname{Pr}(x(\widehat{a}(a)))}{\operatorname{Pr}(z(a))}=\frac{f(x(\widehat{a}))}{f(z(a))} \cdot\left|\frac{\left.x^{\prime}(\widehat{a})\right)}{z^{\prime}(a)}\right| \cdot\left|\widehat{a}^{\prime}(a)\right|
$$

so, denoting $\widehat{I}(\widehat{a})=\{x(\widehat{a}), g(\widehat{a})\}$ and $I(a)=\{z(a), h(a)\}$, the DM's beliefs at the pooled (4-type) information set $\widehat{I}(\widehat{a}) \cup I(a)$ satisfy

$$
\begin{aligned}
\frac{P^{*}}{1-P^{*}} & \equiv \frac{\operatorname{Pr}(\widehat{I}(\widehat{a}))}{\operatorname{Pr}(I(a))}=\frac{\operatorname{Pr}(x(\widehat{a})) \cdot\left(1+\frac{\operatorname{Pr}(g(\widehat{a}))}{\operatorname{Pr}(x(\widehat{a}))}\right)}{\operatorname{Pr}(z(a)) \cdot\left(1+\frac{\operatorname{Pr}(h(a)}{\operatorname{Pr}(z(a)}\right)} \\
& =\frac{\mu(x(\widehat{a}))}{\mu(z(a))} \cdot\left(\frac{\theta_{3} e^{\widehat{a}(a)}-1}{2 e^{a-a_{\gamma}}-1}\right)\left|\widehat{a}^{\prime}(a)\right| \cdot\left(\frac{q_{a}}{p_{a}}\right)
\end{aligned}
$$

(with $p_{a}, q_{a}$ as defined in the first two bullet points; the final term in the first line numerator is $1+\frac{1-p_{a}}{p_{a}}=\frac{1}{p_{a}}$, and similarly final denominator term is $\left.1+\frac{1-q_{a}}{q_{a}} \frac{1}{q_{a}}\right)$. 


\section{References}

[1] Ambrus, A. And S. N. Lu (2010): "Robust almost fully revealing equilibria in multi-sender cheap talk," mimeo.

[2] Andrelini, L. D. Gerardi and R. Lagunoff (2008): "Communication and Learning," mimeo.

[3] Athey, S., and I. Segal (2007): "An Efficient Dynamic Mechanism," Working Paper, Harvard University.

[4] Aumann, R., and S. Hart (2003): "Long Cheap Talk," Econometrica, 71(6), 1619-1660.

[5] Baliga, Sandeep and Tomas Sjostrom (2004): "Arms Races and Negotiations," Review of Economic Studies, 71(2): 351-369.

[6] Baliga, Sandeep and Tomas Sjostrom (2008): "Strategic Ambiguity and Arms Proliferation," Journal of Political Economy, 116, 1023-1057.

[7] Baliga, Sandeep and Tomas Sjostrom (2011): "The Strategy of Manipulating Conflict," mimeo.

[8] Battaglini M. (2002): "Multiple Referrals and Multi-dimensional Cheap Talk." Econometrica 70, 1379-1401.

[9] Bergemann, D. And M. Said (2010): "Dynamic Auctions," Wiley Encyclopedia of Operations Research and Management Science, forthcoming.

[10] Blume, A., O. Board and K. Kawamura (2007): "Noisy Talk", Theoretical Economics $2(4), 395-440$.

[11] Chen, Y., Kartik, N. And J. Sobel, (2008): Selecting Cheap Talk Equilibria, Econometrica, $76,(1), 117-136$.

[12] Crawford, V. and J. Sobel (1982): "Strategic Information Transmission," Econometrica, $50,1431-1451$.

[13] Eso P. And Y.-F. Fong (2008): "Wait and See," mimeo.

[14] Forges, F. And F. Koessler (2008A): "Multistage communication with and without verifiable types", International Game Theory Review, 2008, 10(2), 145-164.

[15] Forges, F. And F. Koessler (2008B): "Long Persuasion Games ", Journal of Economic Theory, 2008, 143(1), 1-35.

[16] Goltsman, M., J. Horner, G. Pavlov, and F. Squintani (2009): "Mediation, Arbitration and Negotiation," Journal of Economic Theory, 144(4), 1397-1420.

[17] Goltsman, M., and G. Pavlov (2008): "How to Talk to Multiple Audiences," Games and Economic Behavior, forthcoming.

[18] Ivanov, M. (2011): "Dynamic Informational Control," mimeo.

[19] Kartik, N., M. Ottaviani, and F. Squintani (2007): "Credulity, Lies, and Costly Talk," Journal of Economic Theory, 134, 93-116

[20] Krishna V. And J. Morgan (2001): "A Model of Expertise," Quarterly Journal of Economics, 116 (2001), 747-75.

[21] Krishna V. And J. Morgan (2004): "The Art of Conversation," Journal of Economic Theory, 117: 147-79. 
[22] Levy, G. And R. Razin (2007): "On The Limits of Communication in Multidimensional Cheap Talk: A Comment," Econometrica, 75 (3), 885-893.

[23] Mathis, J. (2008): "Full Revelation of Information in Sender-Receiver Games of Persuasion," Journal of Economic Theory, 143, 571-584.

[24] Morris, S. (2001): "Political Correctness," Journal of Political Economy, 109, 2, 231-265.

[25] Ottaviani, M. And P. Sorensen (2006): "Professional Advice," Journal of Economic Theory, 126, 1, 120-142.

[26] Ottaviani, M. and P. Sorensen (2006b): "Reputational Cheap Talk," RAND Journal of Economics, 37, 1, 155-175.

[27] Renault, J., Solan, E. And N. Vieille (2011): "Dynamic Sender-Receiver Games," working paper.

[28] Sobel, J. (1985): "The Theory of Credibility," The Review of Economic Studies, LII, 557-573.

[29] Sobel, J. (2008): "Signaling Games," Meyers: Encyclopedia of Complexity and Systems Science (ed.), Springer, forthcoming. 